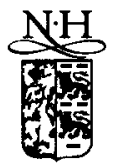

ELSEVIER

Nuclear Physics B 487 (1997) 345-408

\title{
Gauge invariance in simplicial gravity
}

\author{
Herbert W. Hamber ${ }^{1}$, Ruth M. Williams \\ Theoretical Physics Division, CERN, CH-I2IJ Geneva 23, Switzerland
}

Received 24 July 1996; revised 9 December 1996; accepted 20 December 1996

\begin{abstract}
The issue of local gauge invariance in the simplicial lattice formulation of gravity is examined. We exhibit explicitly, both in the weak-field expansion about flat space, and subsequently for arbitrarily triangulated background manifolds, the exact local gauge invariance of the gravitational action, which includes in general both cosmological constant and curvature-squared terms. We show that the local invariance of the discrete action and the ensuing zero-modes correspond precisely to the diffeomorphism invariance in the continuum, by carefully relating the fundamental variables in the discrete theory (the edge lengths) to the induced metric components in the continuum. We discuss mostly the two-dimensional case, but argue that our results have general validity. The previous analysis is then extended to the coupling with a scalar field, and the invariance properties of the scalar field action under lattice diffeomorphisms are exhibited. The construction of the lattice conformal gauge is then described, as well as the separation of lattice metric perturbations into orthogonal conformal and diffeomorphism part. The local gauge invariance properties of the lattice action show that no Faddeev-Popov determinant is required in the gravitational measure, unless lattice perturbation theory is performed with a gauge-fixed action, such as the one arising in the lattice analog of the conformal or harmonic gauges.
\end{abstract}

\section{Introduction}

In the quantization of gravitational interactions one expects non-perturbative effects to play an important role [1]. One formulation available for studying such effects is Regge's simplicial lattice theory of gravity [2]. It is the only lattice model with a local gauge invariance [3], and the only model known to contain gravitons in four dimensions

\footnotetext{
I Permanent address: University of California, Irvine CA 92717, USA.

E-mail address: hamber@cern.ch

${ }^{2}$ Permanent address: DAMTP, Silver Street, Cambridge CB3 9EW, England, UK.

E-mail address: rmw7@damtp.cam.ac.uk;
} 
[4]. One would hope that a number of fundamental issues in quantum gravity, such as the existence of a non-trivial ultraviolet fixed point of the renormalization group in four dimensions and the recovery of General Relativity at large distances, could in principle be addressed in such a model. The presence of a local gauge invariance, which is analogous to the diffeomorphism group in the continuum, makes the model attractive as a regulated theory of gravity [5], while the existence of a phase transition in three [6] and four dimensions [3,7-10] (but not in two [11]) suggests the existence of a (somewhat unusual) lattice continuum limit. The two phases of quantized gravity found in [9], can loosely be described as having in one phase ( $G<G_{c}$, the rough, polymer-like phase)

$$
\left\langle g_{\mu \nu}\right\rangle=0 \text {, }
$$

and in the other phase ( $G>G_{c}$, the smooth phase),

$$
\left\langle g_{\mu \nu}\right\rangle \approx c \eta_{\mu \nu}
$$

with a small negative average curvature (anti-De Sitter space) in the vicinity of the critical point at $G_{c}$. A physically similar two-phase structure was later proposed also in [12]; see also the earlier ideas found in the work of [13]. A discussion of the properties of the two phases characterizing four-dimensional gravity, and of the associated critical exponents, can be found in [9]. For additional recent numerical results we refer the reader to [10], while for some earlier attempts we refer to the work in Refs. $[3,7,8]$. Recently calculations have progressed to the point that a first calculation of the Newtonian potential from the correlation of heavy particle world lines, following the suggestive proposal of [14], seems feasible [15]. The results so far indicate that in the lattice quantum theory of gravity the potential between heavy spinless bodies is attractive, and has roughly the correct heavy mass dependence. In the same work a general scaling theory for gravitational correlations, valid in the vicinity of the fixed point, was put forward. We refer the interested reader to [16], where a more complete set of references to earlier work on Regge gravity can be found. For results with an alternative and complementary approach based on dynamical triangulations, we shall point the reader to the references in [17].

In view of this recent progress it would seem desirable to further elucidate the correspondence between continuum and lattice theories. The weak-field expansion is available to systematically develop this correspondence, and it is well known that such an expansion can be carried out in both formulations. Not unexpectedly, it is technically somewhat more complex in the lattice theory due to the presence of additional vertices, as happens in ordinary lattice gauge theories. In the past most perturbative studies of lattice gravity have focused on the lowest order terms, and in particular the lattice graviton propagators $[4,11,6]$. Recently it has been extended to include the vertex functions, and the results have been used to compute the one-loop amplitudes relevant for the conformal anomaly in two dimensions [18].

One central issue in a regularized theory of quantum gravity is the nature of its invariance properties. Although some discussions of these issues have appeared before, 
no systematic and coherent exposition has been presented yet in the literature. In this paper we address the question of what exactly the local gauge invariance built into Regge's simplicial gravity looks like. Its existence is intimately tied in with the appearance of gravitons (in four dimensions) in the lattice weak-field expansion about a flat background. It need not be emphasized here that local gauge invariance plays a central role in both the classical and quantum formulation of gravity, and its preservation in the lattice theory must therefore be considered of paramount importance. Physically, it expresses the fact that the same physical geometry can be described by equivalent metrics. Classically, it leads for example to the invariance of the infinitesimal line element and the Bianchi identities for the curvature. In the quantum theory it is known to give rise to the Slavnov-Taylor identities for the gravitational Green's functions. As a consequence, one would expect that local gauge transformations should play a central role in the lattice theory as well. This aspect will be therefore the focus of the first part of the paper, where the analog of local gauge transformations on the lattice will be constructed. The requirement of gauge invariance will have implications for both the gravitational measure and the coupling to a scalar field, and we will present in this paper a detailed analysis of its consequences. The second part of this paper will be devoted to a number of relevant applications.

The plan of the paper is as follows. In Section 2 we introduce our notation, describe the choice of lattice structure and the relevant degrees of freedom in the lattice theory, the squared edge lengths. We discuss the discrete actions for the gravitational degrees of freedom, and the relationship to their well-known continuum counterparts. In Section 3 we move on to the lattice weak-field expansion, and discuss in detail the two-dimensional case (with cosmological and curvature-squared terms). We exhibit explicitly the gauge zero-modes and their corresponding eigenvectors, which are shown to correspond precisely to local gauge transformations in the continuum. We then compute explicitly and analytically the zero-modes for fluctuations about a non-flat background (the tetrahedral, octahedral and icosahedral tessellations of the two-sphere), and show that the counting of the zero-modes is indeed consistent with the expectation from the continuum theory. We then give further arguments supporting the identification of the zero-modes with the diffeomorphisms in the continuum, which we argue is valid in any dimension. In Section 4 we extend the previous analysis to arbitrary curved backgrounds and show explicitly the persistence of a local gauge invariance for the area, curvature and curvature-squared terms. In Section 5 we introduce a scalar field coupled invariantly to the gravitational degrees of freedom. We again exhibit its invariance properties under local gauge variations of the squared edge lengths, at least for sufficiently smooth scalar field configurations, by working out the concrete case of background lattices which are close to either equilateral or square. We then discuss the more general case of arbitrary background lattices, and the construction of the energy-momentum tensor for the scalar field. Section 6 discusses the implications of the preceding results for the lattice gravitational measure, and we give arguments that the lattice measure is essentially unique, up to local volume factors. We will argue therefore that the lattice measure is essentially no less unique than the original continuum (DeWitt) measure. In Section 7 we 
consider the possibility of introducing a gauge-fixing term in the lattice action, in order to remove the gauge zero-modes of the gravitational action and subsequently perform perturbative calculations, and in close analogy with the procedure followed in the usual continuum perturbation theory. As an example, we discuss the explicit construction of the lattice conformal gauge, starting from an arbitrary configuration of squared edge lengths. Finally, Section 8 contains some concluding remarks.

\section{The discretized theory}

In this section we will briefly review the construction of the action describing the gravitational field on the lattice, and define the necessary notation used later in the paper. In concrete examples we will often refer, because of its simplicity, to the twodimensional case, where a number of results can be derived easily and transparently. But in a number of instances here, and throughout the paper, important aspects of the discussion and of the conclusions will be quite general, and not restricted to specific aspects of the two-dimensional case.

In simplicial gravity the elementary building blocks for $d$-dimensional space-time are simplices $\sigma^{d}$ of dimension $d$. A 0 -simplex is a point, a 1-simplex is an edge and a 2-simplex is a triangle. A $d$-simplex is a $d$-dimensional object with $d+1$ vertices and $d(d+1) / 2$ edges connecting them. Each simplex in turn contains $\left(\begin{array}{l}d+1 \\ k+1\end{array}\right)$ sub-simplices $\sigma^{k}$ of dimension $k$. Thus in two dimensions we shall consider here a fixed closed simplicial two-manifold consisting of $N_{0}$ vertices, $N_{1}$ edges and $N_{2}$ triangles, joined in such a way that each point has a neighborhood homeomorphic to the interior of a two-dimensional sphere. A simplicial geometry is then specified by the assignment of squared edge lengths $l_{i}^{2}, i=1, \ldots, N_{1}$, and a flat Riemannian metric can be assigned to the interior regions of the simplices in a way that is consistent with the edge length values. Further restrictions arise from the fact that the triangle inequalities (and their higher-dimensional analogs in $d$ dimensions) have to be satisfied.

The correspondence between squared edge lengths and an assigned continuum metric field can be made more precise, with the identification

$$
l_{a b}=\int_{\tau(a)}^{\tau(b)} d \tau \sqrt{g_{\mu \nu}(x(\tau)) \frac{d x^{\mu}}{d \tau} \frac{d x^{\nu}}{d \tau}}=\sqrt{g_{\mu \nu} l_{a b}^{\mu} l_{a b}^{\nu}},
$$

where $l_{a b}$ is the length of the edge connecting neighboring points $a$ and $b$. For a given set of edge lengths, the metric $g_{\mu \nu}(x)$ has initially support on the edges only. ${ }^{3}$ For a

\footnotetext{
${ }^{3}$ The above identification parallels an analogous correspondence used sometimes in ordinary lattice gauge theories, where the $S U(n)$ matrix-valued lattice field $A_{n \mu}$ has support only on the links of a hypercubic lattice, $U_{n \mu} \equiv e^{i a A_{n \mu}}=P \exp \left(i a \int_{n}^{n+\mu} d x^{\mu} A_{\mu}(x)\right)$. This definition is a convenient starting point for performing perturbation theory and defining the lattice Feynman rules. For the same construction in perturbative simplicial gravity see Ref. [18].
} 
metric that is constant inside each simplex, $l_{i}^{2}=g_{\mu \nu} l_{i}^{\mu} l_{i}^{\nu}$, where $i$ labels the edge from $a$

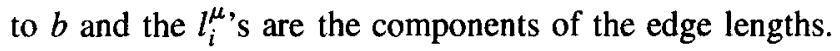

\subsection{Lattice structure}

In two dimensions quantum gravity can be defined on a two-dimensional surface consisting of a network of flat triangles. The underlying lattice may be constructed in a number of ways. Points may be distributed randomly on the surface and then joined to form triangles according to some algorithm. In such lattices the coordination number at each vertex can be kept fixed (quenched random lattice), or allowed to vary (annealed random lattice), by considering it as an additional, dynamical variable of the model. An alternative procedure is to start with a regular lattice, like a regular tessellation of the two-sphere, or a lattice of squares divided into triangles by drawing in parallel sets of diagonals, and then allow the edge lengths to vary, which will give rise to curvature localized on the vertices. It should be emphasized that for arbitrary assignments of edge lengths, consistent with the imposition of the triangle inequalities constraints, such a lattice is in general far from regular and resembles more a random lattice.

The incidence matrix, which provides the information on which edges are adjacent, and fixes therefore the local coordination number $q_{i}$, describes the topology of the manifold. It can be chosen to correspond to a fixed regular or to a fixed random lattice. But one word of caution should be spent here on the terminology. Since the edge lengths are dynamical variables, the lattice is in fact random in either case: contrary to a fixed regular lattice (such as the square or triangular one in two dimensions), there are a priori no preferred directions even for a lattice with fixed coordination number, as neighboring points can have any relative orientation as long as they are consistent with the triangle inequalities and their higher-dimensional analogs. Universality arguments would then suggest that the choice of local coordination number should not affect the large-distance limit of the model, and a number of explicit calculations on random lattices have shown to some extent that this is indeed the case $[19,20]$.

In the following we will often narrow down the discussion and be even more specific, and usually think of the "regular" lattice as consisting of a network of triangles with a fixed coordination number of six, $q_{i}=6$, although many of the results in this work are quite general and do not rely on the specific choice of local coordination numbers.

Quenched random lattices, where the local coordination number $q_{i}$ (which is the number of edges meeting at $i$ ) is random but fixed, were considered in $[21,22,19,23]$. For such Poissonian random lattices, the average coordination number is also $q=6$ in two dimensions, independent of the topology. This follows from the expression for the Euler characteristic $\chi=N_{0}-N_{1}+N_{2}$ with $2 N_{1}=3 N_{2}=\sum_{i} q_{i}$ in two dimensions, which gives for large $N_{0}$

$$
q \equiv \lim _{N_{0} \rightarrow \infty} \frac{\sum_{i} q_{i}}{\sum_{i} 1}=6,
$$


irrespective of the value of $\chi$, as well as $N_{1}=3 N_{0}$ and $N_{2}=2 N_{0}$. In general on such random lattices one does not have, strictly speaking, translational or rotational invariance for a fixed assignment of edge lengths. The latter only hold on the average. Explicit calculations confirm that this is indeed the case, at least in two dimensions [19,24,25].

When the edge lengths are allowed to fluctuate one would expect the situation to be different, since now locally there are no preferred directions any more, as the lattice structure fluctuates from edge length configuration to edge length configuration. In principle, one can allow the local coordination number to change (annealed random lattice) by re-linking neighboring vertices, although there is no unique algorithm to do so which preserves the geometry. In this case the coordination number fluctuation $\delta q_{i}=q_{i}-6$ becomes an additional dynamical variable, and is indeed the only dynamical variable in the so-called dynamical triangulations. Randomness can be shown to be a relevant perturbation in two dimensions, changing the universality class already for flat surfaces. We shall not consider dynamical random lattices here any further, as we are interested in discretizations for gravity coupled to matter which maintain the crucial property of reducing to the ordinary, known flat-space field theories in the limit of zero local curvatures. A review of the properties of random lattices and their relation to matrix models in two dimensions can be found in [26].

\subsection{Degrees of freedom}

The elementary degrees of freedom on the lattice are the squared edge lengths, with the correspondence between continuum and lattice degrees of freedom given locally by

$$
\left\{g_{\mu \nu}(x)\right\}_{x \in \mathcal{M}} \rightarrow\left\{l_{i}^{2}\right\}_{i=1, \ldots, N_{1}},
$$

where the index $i$ ranges over all $N_{1}$ edges in the lattice. In general the dynamical lattice will give rise to some average lattice spacing $a_{0}=\left[\left\langle l^{2}\right\rangle\right]^{1 / 2}$, which in turn will naturally supply the ultraviolet cutoff that is needed to define the quantum theory. An important difference with ordinary lattice field theories lies in the fact that the momentum cutoff $\Lambda=1 / l_{0}$ is not determined a priori, but follows instead from the dynamics (i.e. from the lattice action and lattice measure). The dynamical cutoff turns out to be determined mostly by the cosmological constant term and the measure factor [9]. Furthermore, for finite volumes the lattice theory will have a finite number of degrees of freedom $N$, and will therefore inherit an infrared cutoff of the order of $1 / L$, where $L$ is the physical linear extent of the lattice.

In the discrete case all the metric information on the piecewise linear space is contained in the values of the edge lengths. As already emphasized by Regge, and in accordance with the usual view of lattice discretization of continuum field theories, the discrete manifold $L$ is thought of as an approximation to some continuum manifold $S$ (as illustrated in Fig. 1). In the limit as the average lattice spacing $a_{0}$ is sent to zero, the original continuum theory is recovered. In four dimensions it has been rigorously proven, for the Einstein-Regge action, that if a piecewise flat space approximates a smooth space in a suitable sense, then the corresponding curvatures are close in the 


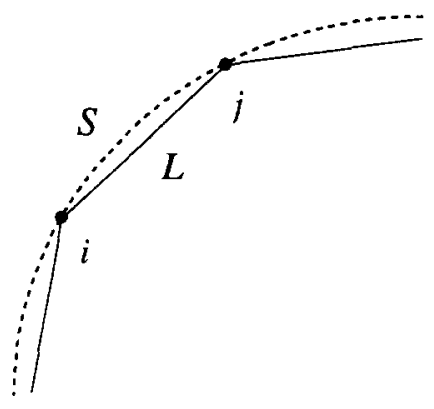

Fig. 1. Piecewise linear space $L$ as an approximation to a smooth $d$-dimensional enveloping surface $S$.

sense of measures [27]; see also the results of [29]. In general the expectation is that the lattice and continuum theory will differ by higher order corrections, with the two actions related to each other by

$$
I_{L}\left(l^{2}\right)=I_{C}\left(g_{\mu \nu}\right)+a_{0} \delta I+a_{0}^{2} \delta^{2} I+\ldots
$$

All corrections can in principle systematically be evaluated by the standard procedure of replacing the finite differences which appear in the lattice action by derivatives, for example according to the formula

$$
\frac{g\left(n+a_{0}\right)-g\left(n-a_{0}\right)}{2 a_{0}}=g^{\prime}(n)+\frac{1}{6} a_{0}^{2} g^{\prime \prime \prime}(n)+O\left(a_{0}^{4} g^{(5)}(n)\right) .
$$

It should be noted that higher order corrections are expected to involve higher derivatives of the metric. The above expansion procedure can be thought of being equivalent to introducing a continuum metric on the piecewise linear manifold, and expand in the difference between the continuum and the piecewise linear metric.

This interpretation is analogous to the situation in ordinary lattice gauge theories, where the lattice gauge fields $U_{n \mu}$ are defined on the links only; the continuum fields $A_{\mu}(x)$ can then be reconstructed by some suitable interpolation to the interior regions of the lattice. It is of course possible to endow the piecewise linear space with a continuum metric $g_{\mu \nu}(x)$ which is defined everywhere, including the interiors of the simplices. In this case a continuum curvature $R_{\mu \nu \rho \sigma}(x)$ can be defined as well, but since the interior of the simplices is flat, the curvature acquires delta-function singularities on the hinges where the discrete curvature resides. While such a description can be useful in certain circumstances, it has also some drawbacks, which have led to considerable confusion in some of the literature. The obvious ones are that the resulting model is no longer an ultraviolet regulator for the continuum theory, as space-time has become continuous again. Furthermore the fields are singular, due to the delta-function type singularities in the curvature, and the number of degrees of freedom is no longer finite due to the re-introduction of a continuum metric. In this formalism new divergences appear, which have to be regulated by some ad-hoc procedure such as the smoothing out of conical singularities, and lead to difficulties in defining higher order invariant operators such as the ones containing curvature-squared terms [7]. 
This point of view, while certainly legitimate in discussing some classical aspects of the theory, is therefore in our opinion not useful in describing a regulated theory of quantum gravity. It leads instead to a string of paradoxical results when lattice and continuum language are mixed together, and can be especially misleading when discussing such subtle issues as the gravitational functional integration measure.

It should be emphasized here that in the following we shall restrict our attention almost exclusively to the lattice theory, which is defined in terms of its lattice degrees of freedom only. Since it is our purpose to describe an ultraviolet regulated theory of quantum gravity, we shall follow the usual procedure followed in discussing lattice field theories, and discuss the model exclusively in terms of its primary, lattice degrees of freedom: the squared edge lengths. As such, the theory will not require any additional ad-hoc regulators. Below we shall discuss further at length a number of issues related to the precise correspondence between the lattice degrees of freedom and the continuum ones, the local gauge invariance of the lattice action (which gives rise in the quantum theory the lattice analogs of the Taylor-Slavnov identities) and the need for (or lack of) gauge fixing.

\subsection{Curvature and discretized action}

The construction of the lattice action starts from the definition of the elementary building blocks for space-time, the $n$-dimensional simplices. Consider an $n$-dimensional simplex with vertices $1,2,3, \ldots n+1$ and square edge lengths $l_{12}^{2}=l_{21}^{2}, \ldots$ Its vertices are specified by a set of vectors $e_{0}=0, e_{1}, \ldots e_{n}$ in flat Euclidean space. The matrix

$$
g_{i j}=\boldsymbol{e}_{i} \cdot \boldsymbol{e}_{j},
$$

with $1 \leqslant i, j \leqslant n$, is positive definite. In terms of the edge lengths $l_{i j}=\left|\boldsymbol{e}_{i}-\boldsymbol{e}_{j}\right|$ (see Fig. 2) it is given by

$$
g_{i j}\left(l^{2}\right)=\frac{1}{2}\left[l_{0 i}^{2}+l_{0 j}^{2}-l_{i j}^{2}\right] .
$$

The volume of a general $n$-simplex is then given by an $n$-dimensional generalization of the well-known formula for the volume of a tetrahedron,

$$
V_{n}\left(l^{2}\right)=\frac{1}{n !} \sqrt{\operatorname{det} g_{i j}\left(l^{2}\right)} .
$$

Conversely, in order to obtain a simplex for an arbitrary assignment of edge lengths, the generalization to higher dimensions of the triangle inequalities require that $V_{n}^{(i)}\left(l^{2}\right) \geqslant 0$, with $n=1, \ldots, d$ and $i=1, \ldots, N_{n}$ be satisfied for every edge, triangle, tetrahedron etc. in the lattice. This can be stated equivalently by requiring

$$
\operatorname{det} g_{i j}\left(l^{2}\right)>0
$$

for every sub-determinant of the highest dimension det $g_{i j}$. In $d$ dimensions the matrix $g_{i j}$ has $d(d+1) / 2$ components, just as there are $d(d+1) / 2$ components for the metric $g_{\mu \nu}(x)$ per space-time point in the continuum. 


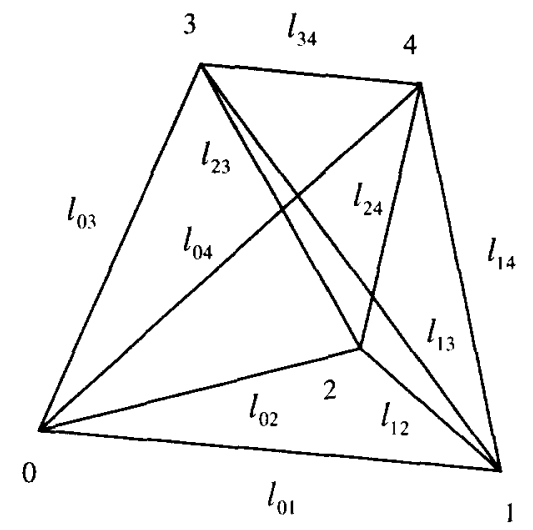

Fig. 2. Assignments of edge lengths for a four-dimensional simplex.

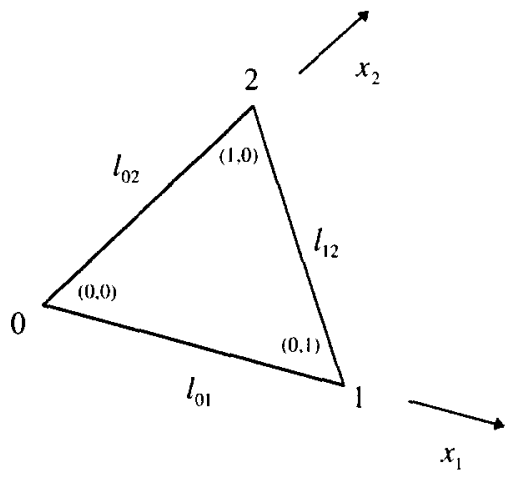

Fig. 3. Assignments of edge lengths and natural coordinates for a triangle.

In this paper we shall often refer to the two-dimensional case. In two dimensions one has simply

$$
g_{i j}\left(l^{2}\right)=\left(\begin{array}{cc}
l_{01}^{2} & \frac{1}{2}\left(l_{01}^{2}+l_{02}^{2}-l_{12}^{2}\right) \\
\frac{1}{2}\left(l_{01}^{2}+l_{02}^{2}-l_{12}^{2}\right) & l_{02}^{2}
\end{array}\right),
$$

and therefore

$$
\operatorname{det} g_{i j}\left(l^{2}\right)=\frac{1}{4}\left[2\left(l_{01}^{2} l_{02}^{2}+l_{02}^{2} l_{12}^{2}+l_{12}^{2} l_{01}^{2}\right)-l_{01}^{4}-l_{02}^{4}-l_{12}^{4}\right]
$$

and

$$
\sqrt{\operatorname{det} g_{i j}\left(l^{2}\right)}=2 A_{T}\left(l^{2}\right),
$$

where $A_{T}\left(l^{2}\right)$ is the area of the given triangle (see Fig. 3).

In simplicial gravity the curvature is concentrated on the hinges, which are subspaces of dimensions $d-2$, and is entirely determined from the assignment of the edge lengths. In two dimensions the hinges correspond to the vertices and $\delta_{h}$, the deficit angle at a hinge, is defined by 


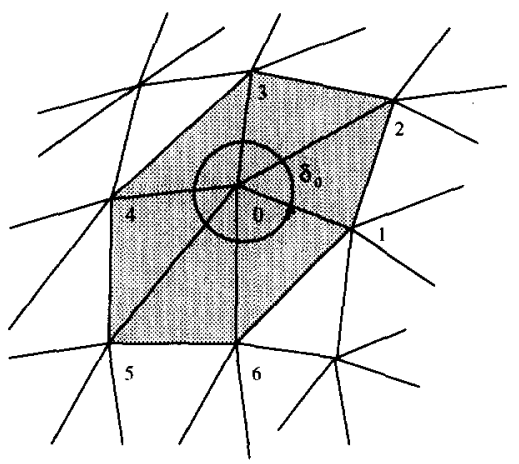

Fig. 4. In two dimensions the computation of the deficit angle $\delta_{0}$ at the vertex 0 involves the values for the edge lengths associated with the shaded triangles.

$$
\delta_{h}=2 \pi-\sum_{\substack{\text { triangles }, \\ \text { mecting at } h}} \theta_{t},
$$

where $\theta_{t}$ is the dihedral angle associated with the triangle $t$ at the vertex $h$ (see Fig. 4). In $d$ dimensions several $d$-simplices meet on a $(d-2)$-dimensional hinge, and the deficit angle is defined by

$$
\delta_{h}\left(l^{2}\right)=2 \pi-\sum_{\substack{d-\text { simplices } \\ \text { meecing on } h}} \theta_{d}\left(l^{2}\right),
$$

where $\theta_{d}$ is the dihedral angle in $d$ dimensions. The sine of the dihedral angle can be computed from the well-known formula

$$
\sin \theta_{d}\left(l^{2}\right)=\frac{d}{d-1} \frac{V_{d} V_{d-2}}{V_{d-1} V_{d-1}^{\prime}},
$$

where $V_{d-2}$ is the volume of the hinge, $V_{d}$ is the volume of the $d$-simplex, and $V_{d-1}$, $V_{d-1}^{\prime}$ the volumes of the two $(d-1)$-dimensional faces that meet on the hinge. A general derivation of these formulae can be found in [5], with some additional results in [7]. Since the sine does not uniquely determine the angle, it can be useful to obtain an expression for the cosine of the dihedral angle, which can be found in [7]. In two dimensions the dihedral angle is given by

$$
\cos \theta_{d}=\frac{l_{01}^{2}+l_{02}^{2}-l_{12}^{2}}{2 l_{01} l_{02}} .
$$

It is useful to introduce a dual lattice following, for example, the Dirichlet-Voronoi cell construction, which consists in introducing the perpendicular bisectors of the edges in each triangle and joining the resulting vertices. This provides for a natural subdivision of the original lattice in a set of non-overlapping exhaustive cells, and has a natural generalization to higher dimensions. It is easy to see that the vertices of the original lattice then reside on circumscribed circles, centered on the vertices of the dual lattice. For the vertex 0 the dihedral dual volume contribution, shown in Fig. 5, is given by 


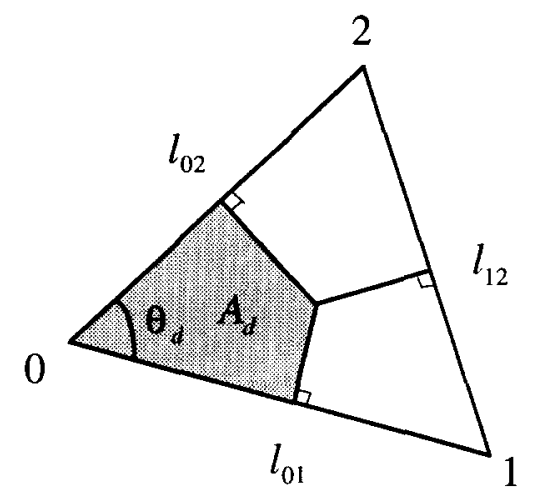

Fig. 5. Dual area $A_{d}$ associated with vertex 0 , and the corresponding dihedral angle $\theta_{d}$.

$$
A_{d}\left(l^{2}\right)=\frac{1}{32 A}\left[l_{12}^{2}\left(l_{01}^{2}+l_{02}^{2}\right)-\left(l_{01}^{2}-l_{02}^{2}\right)^{2}\right] \text {. }
$$

It is clear that the above subdivision is not unique. Alternatively, one can introduce a baricenter for each triangle, defined as the point equidistant from all three vertices, and again join the resulting vertices. The vertices of the original lattice then reside on inscribed circles, centered on the vertices of the dual lattice. The baricentric dihedral volume is simply given by

$$
A_{d}\left(l^{2}\right)=A / 3 .
$$

In general, if the original lattice has local coordination number $q_{i}$ at the site $i$, then the dual cell centered on $i$ will have $q_{i}$ faces. A fairly complete set of formulae for dual volumes relevant for lattice gravity and their derivation can be found in [7]. In the following we shall refer to the Voronoi cell construction as the "dual subdivision", while we will call the baricentric cell construction the "baricentric subdivision".

Two-dimensional Einstein gravity is trivial because the Einstein action is constant and the Ricci tensor vanishes identically. When a cosmological constant term and a curvature-squared term are included in the action,

$$
I=\int d^{2} x \sqrt{g}\left[\lambda-k R+a R^{2}\right],
$$

the classical solutions have constant curvature with $R= \pm \sqrt{\lambda / a}$ (there being no real solutions for $\lambda<0$ ). Thus the theory with the Einstein action and a cosmological constant is metrically trivial, having neither dynamical degrees of freedom nor field equations. On the other hand, the functional measure can lead to a non-trivial effective action. However, for a system with fixed topology, the only non-classical aspects of $1+1$-dimensional gravity are fluctuations in the local volumes $\sqrt{g(x)}$.

The Einstein action for a two-dimensional simplicial lattice is given by [2]

$$
\int d^{2} x \sqrt{g} R \longrightarrow 2 \sum_{\text {hinges } h} \delta_{h}
$$




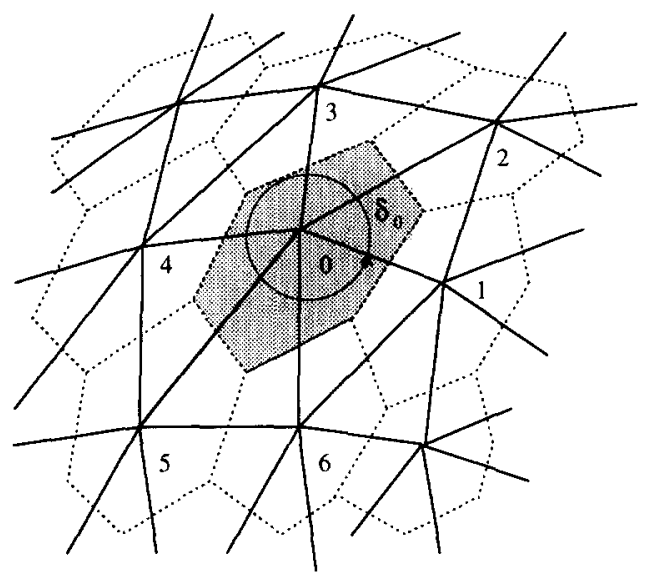

Fig. 6. Original simplicial lattice (continuous lines) and dual lattice (dotted lines) in two dimensions. The shaded region corresponds to the dual area associated with vertex 0 .

According to the Gauss-Bonnet theorem the Einstein action in two dimensions is equal to $4 \pi$ times the Euler characteristic of the surface. The same result is true on the lattice, with $\sum_{h} \delta_{h}=2 \pi \chi$, where $\chi$ is the Euler characteristic. It is a constant provided we consider, as we shall do below, surfaces with a fixed topology.

A cosmological constant term can be included in the action in the form

$$
\lambda \int d^{2} x \sqrt{g} \longrightarrow \lambda \sum_{\text {triangles } t} A_{t},
$$

where $A_{t}$ is the area of triangle $t$. Equivalently one may subdivide the triangles into areas associated with each hinge $A_{h}$ and use the expression

$$
\lambda \sum_{\text {hinges } h} A_{h} .
$$

For the baricentric subdivision one has simply

$$
A_{h}=\frac{1}{3} \sum_{\substack{\text { triangles I } \\ \text { meecingat } h}} A_{t} .
$$

$A_{h}$ can also be taken to be the area of the cell surrounding $h$ in the dual lattice (see Fig. 6), with

$$
A_{h}=\sum_{\substack{\text { Uizangles o } \\ \text { meetiog at } h}} A_{d},
$$

with the dual area contribution for each triangle $A_{d}$ given in Eq. (2.17).

In two dimensions the Weyl tensor vanishes identically, while the other curvaturesquared terms are all proportional to each other,

$$
R_{\mu \nu \rho \sigma} R^{\mu \nu \rho \sigma}=\frac{1}{2} R_{\mu \nu} R^{\mu \nu}=R^{2} .
$$


One therefore needs only one term quadratic in the curvature for the lattice action. Using the requirements that it be a sum over hinges (the only places where the curvature is non-zero), that it be quadratic in the deficit angle, and that it have the correct dimension (length) ${ }^{-2}$, one is led to the unique expression

$$
\int d^{2} x \sqrt{g} R^{2} \longrightarrow 4 \sum \frac{\delta_{h}^{2}}{A_{h}} .
$$

It can be shown that this formula is exact for all regular tessellations of the two-sphere, in the sense that the discrete lattice expression does not depend on the how fine the tessellation is, once the area of the surface is kept fixed [7].

The lattice action corresponding to pure gravity is then

$$
I\left(l^{2}\right)=\sum_{h}\left[\lambda A_{h}-2 k \delta_{h}+4 a \frac{\delta_{h}^{2}}{A_{h}}\right],
$$

which can be written equivalently as

$$
I\left(l^{2}\right)=\sum_{h} V_{h}\left[\lambda-k R_{h}+a R_{h}^{2}\right]
$$

with the two-dimensional volume element $V_{h}=A_{h}$, and the local curvature given by $R_{h}=2 \delta_{h} / A_{h}$. In the limit of small fluctuations around a smooth background, $I\left(l^{2}\right)$ corresponds to the continuum action

$$
I[g]=\int d^{2} x \sqrt{g}\left[\lambda-k R+a R^{2}\right] .
$$

For a manifold of fixed topology the term proportional to $k$ can be dropped, since $\sum_{h} \delta_{h}=2 \pi \chi$, where $\chi$ is the Euler characteristic. The curvature-squared leads to nontrivial interactions in two dimensions, although the resulting theory is not unitary. In the next section we shall discuss properties of the above action in the weak-field expansion about flat space, and later about an arbitrary lattice manifold.

Arguments based on perturbation theory about two dimensions (where the gravitational coupling is dimensionless and the Einstein theory becomes renormalizable) suggest that there should be no non-trivial ultraviolet fixed point of the renormalization group in two dimensions. Explicit calculations in the lattice theory have shown conclusively that this is indeed the case in the absence of matter [11,30-33]. The equations of motion for pure gravity in two dimensions then follow from the variation

$$
\delta I[g]=\frac{1}{2} \int d^{2} x \sqrt{g}\left[\lambda-a R^{2}\right] g^{\mu \nu} \delta g_{\mu \nu}=0,
$$

and read

$$
\frac{a}{2} R^{2} g_{\mu \nu}-\frac{\lambda}{2} g_{\mu \nu}=0,
$$

or, in contracted form, $R^{2}=\lambda / a$. For an arbitrary gauge variation of the metric, 


$$
\delta g_{\mu \nu}(x)=-g_{\mu \lambda}(x) \partial_{\nu} \chi^{\lambda}(x)-g_{\lambda \nu}(x) \partial_{\mu} \chi^{\lambda}(x)-\partial_{\lambda} g_{\mu \nu}(x) \chi^{\lambda}(x),
$$

one obtains after an integration by parts, and using the fact that the gauge function $\chi^{\lambda}$ is arbitrary (and that $\left.\left(g^{\mu \nu}\right)_{; \nu}=0\right)$,

$$
\left(R^{2} g^{\mu \nu}\right)_{; \nu}=0
$$

This is the two-dimensional analog of the (contracted) Bianchi identity. Since the squared edge lengths are the primary degrees of freedom, the corresponding lattice field equations of motion are obtained, in any dimension, from

$$
\frac{\partial I\left[l^{2}\right]}{\partial l_{i}^{2}}=0
$$

Already in the two-dimensional case they are rather unwieldy when written out explicitly, and will not be recorded here.

A candidate for the discrete analog of the two-dimensional Bianchi identity is simply

$$
\sum_{h(i)} \delta_{h}\left(l_{i}^{2}+\delta l_{i}^{2}\right)-\sum_{h(i)} \delta_{h}\left(l_{i}^{2}\right)=0,
$$

where the sum includes the four hinges $h$ belonging to the two triangles bordering the edge $i$, and $\delta l_{i}^{2}$ represents a variation of the edges meeting at the vertex $h$. By considering gauge variations of the edge lengths in higher dimensions, the corresponding exact lattice Bianchi identities can in principle be written down. Some further discussion of the Bianchi identities in higher dimensions can be found in the second reference in [4].

\section{Lattice weak-field expansion and zero-modes}

One of the simplest problems which can be studied analytically in the continuum as well as on the lattice is the analysis of small fluctuations about some classical background solution. In the continuum, the weak-field expansion is often performed by expanding the metric and the action about flat Euclidean space

$$
g_{\mu \nu}(x)=\delta_{\mu \nu}+\kappa h_{\mu \nu}(x) .
$$

In four dimensions $\kappa=\sqrt{32 \pi G}$, which shows that the weak-field expansion there corresponds to an expansion in powers of $G$. In two dimensions this is no longer the case and the relation between $\kappa$ and $G$ is lost; instead one should regard $\kappa$ as a dimensionless expansion parameter which is eventually set to one, $\kappa=1$, at the end of the calculation. The procedure will be sensible as long as wildly fluctuating geometries are not important in two dimensions (on the lattice or in the continuum). The influence of the latter configurations can only be studied by numerical simulations of the full path integral $[11,30]$. 
In the lattice case the weak-field calculations can be carried out in three- [6] and four- [4] dimensional flat background space with the Regge-Einstein action. One finds that the Regge gravity propagator indeed agrees exactly with the continuum result [34] in the weak-field limit. As a result, the existence of gravitational waves and gravitons in the discrete lattice theory has been established (indeed it is the only lattice theory of gravity for which such a result has been obtained ${ }^{4}$ ).

The weak-field expansion about flat space is relevant for the continuum limit of the lattice quantum theory. Consider a simplicial lattice approximation to a given continuum manifold. For an arbitrary continuum manifold, one can envision a triangulation which is successively refined by making the simplices and the corresponding edge lengths smaller and smaller. As the average lattice spacing is reduced, the curvature on the scale of the lattice spacing becomes eventually sufficiently small that the simplicial manifold can be regarded as being locally close to flat. In this limit the curvature is small on the scale of the local volume, and in two dimensions one has

$$
\text { |curvature }\left.\right|_{h} \equiv\left|\frac{\delta_{h}}{A_{h}}\right| \ll(\text { volume })_{h}^{-1} \equiv \frac{1}{A_{h}} \quad \text { or } \quad\left|\delta_{h}\right| \ll 1 .
$$

In such regions, which become larger and larger in size as the lattice spacing is reduced, one can meaningfully apply the weak-field expansion about flat space, which becomes only an approximation when it is truncated to any finite order.

In the following we shall consider in detail only the two-dimensional case, although similar calculations can in principle be performed in higher dimensions, with considerable more algebraic effort. In the pure gravity case the Einstein-Regge action is a topological invariant in two dimensions, and one has to consider the next non-trivial invariant contribution to the action. We shall therefore consider a two-dimensional lattice with the higher derivative action of Eq. (2.27) and $\lambda=0$,

$$
I\left(l^{2}\right)=4 a \sum_{\text {hinges } h} \frac{\delta_{h}^{2}}{A_{h}} .
$$

The weak-field expansion for such a term has largely been done in [11], and we will first recall here the main results. Since flat space is a classical solution for such an $R^{2}$ type action, one can take as a background space a network of unit squares divided into triangles by drawing in parallel sets of diagonals (see Fig. 7). This is one of an infinite number of possible choices for the background lattice, and a rather convenient one. Physical results should in the end be insensitive to the choice of the background lattice used as a starting point for the weak-field expansion. Opposite edges of the network are supposed to be identified so that the lattice acquires the topology of a torus. (In

\footnotetext{
${ }^{4}$ A discretization of the edge lengths, and therefore of the curvatures, as advocated in some models for lattice gravity, can be considered, the dynamical triangulations being one specific example. This procedure leads obviously to a loss of the graviton excitation, at least in the weak-field expansion. In ordinary non-abelian lattice gauge theories, models based on discrete subgroups of $S U(N)$ have an artificial freezing transition at finite coupling and no lattice continuum limit, and do not seem to represent a useful discretization of the original continuum theory [35].
} 
$(0,1)$

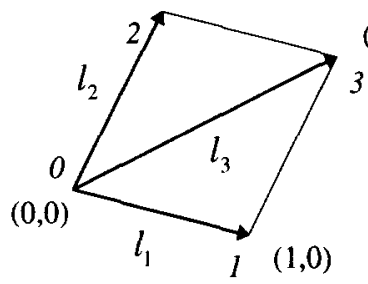

Fig. 7. Notation for the weak-field expansion about the rigid square lattice.

the following we will be concerned with local properties of the action, and the detailed nature of the boundary conditions will play only a marginal role.)

It is also convenient to use the binary notation for vertices described in Ref. [4]. As discussed in the previous section, the edge lengths on the lattice correspond to the metric degrees of freedom in the continuum. The edge lengths are thus allowed to fluctuate around their flat-space values,

$$
l_{i}=l_{i}^{0}\left(1+\epsilon_{i}\right),
$$

with $l_{1}^{0}=l_{2}^{0}=1$ and $l_{3}^{0}=\sqrt{2}$ for our choice of lattice. The second variation of the action is then expressed as a quadratic form in the $\epsilon$ 's,

$$
\delta^{2} I=4 a \sum_{i j} \epsilon_{i} M_{i j} \epsilon_{j} .
$$

The properties of $M_{i j}$ are best studied by going to momentum space. One assumes that the fluctuation $\epsilon_{i}$ at the point $i, j$ steps in one coordinate direction and $k$ steps in the other coordinate direction from the origin, is related to the corresponding $\epsilon_{i}$ at the origin by

$$
\epsilon_{i}^{(j+k)}=\omega_{1}^{j} \omega_{2}^{k} \epsilon_{i}^{(0)},
$$

where $\omega_{i}=e^{-i k_{i}}$ and $k_{i}$ is the momentum in the direction $i$. The matrix $M$ then reduces to a $3 \times 3$ matrix $M_{\omega}$ with components given by [11]

$$
\begin{aligned}
& \left(M_{\omega}\right)_{11}=2+\omega_{1}-2 \omega_{2}-2 \omega_{1} \omega_{2}+\omega_{1} \omega_{2}^{2}+\text { c.c. } \\
& \left(M_{\omega}\right)_{12}=2-\omega_{1}-\bar{\omega}_{2}-\omega_{1} \omega_{2}-\bar{\omega}_{1} \bar{\omega}_{2}-\omega_{1}^{2}-\bar{\omega}_{2}^{2}+\omega_{1}^{2} \omega_{2}+\bar{\omega}_{1} \bar{\omega}_{2}^{2}+2 \omega_{1} \bar{\omega}_{2}, \\
& \left(M_{\omega}\right)_{13}=2\left(-1+2 \omega_{1}-\bar{\omega}_{1}+\omega_{2}-\bar{\omega}_{2}-\omega_{1} \omega_{2}+2 \bar{\omega}_{1} \bar{\omega}_{2}+\bar{\omega}_{2}^{2}-\bar{\omega}_{1} \bar{\omega}_{2}^{2}-\omega_{1} \bar{\omega}_{2}\right), \\
& \left(M_{\omega}\right)_{33}=4\left(2-2 \omega_{1}-2 \omega_{2}+\omega_{1} \omega_{2}+\bar{\omega}_{1} \omega_{2}+\text { c.c. }\right)
\end{aligned}
$$

with the other components easily obtained by symmetry. For small momenta $M_{\omega}$ takes the form

$$
\boldsymbol{M}_{\omega}=l^{4}\left(\begin{array}{ccc}
k_{2}^{2}\left(k_{1}+k_{2}\right)^{2} & k_{1} k_{2}\left(k_{1}+k_{2}\right)^{2} & -2 k_{1} k_{2}^{2}\left(k_{1}+k_{2}\right) \\
k_{1} k_{2}\left(k_{1}+k_{2}\right)^{2} & k_{1}^{2}\left(k_{1}+k_{2}\right)^{2} & -2 k_{1}^{2} k_{2}\left(k_{1}+k_{2}\right) \\
-2 k_{1} k_{2}^{2}\left(k_{1}+k_{2}\right) & -2 k_{1}^{2} k_{2}\left(k_{1}+k_{2}\right) & 4 k_{1}^{2} k_{2}^{2}
\end{array}\right)+O\left(k^{5}\right) .
$$


The change of variables

$$
\epsilon_{1}^{\prime}=\epsilon_{1}, \quad \epsilon_{2}^{\prime}=\epsilon_{2}, \quad \epsilon_{3}^{\prime}=\frac{1}{2}\left(\epsilon_{1}+\epsilon_{2}\right)+\epsilon_{3}
$$

leads for small momenta to the matrix $M_{\omega}^{\prime}$ given by

$$
M_{\omega}^{\prime}=l^{4}\left(\begin{array}{ccc}
k_{2}^{4} & k_{1}^{2} k_{2}^{2} & -2 k_{1} k_{2}^{3} \\
k_{1}^{2} k_{2}^{2} & k_{1}^{4} & -2 k_{1}^{3} k_{2} \\
-2 k_{1} k_{2}^{3} & -2 k_{1}^{3} k_{2} & 4 k_{1}^{2} k_{2}^{2}
\end{array}\right)+O\left(k^{5}\right) .
$$

This expression is identical to what one obtains from the corresponding weak-field limit in the continuum theory. To see this, define as usual the small fluctuation field $h_{\mu \nu}$ about flat space by setting

$$
g_{\mu \nu}=\delta_{\mu \nu}+h_{\mu \nu} .
$$

In two dimensions one has

$$
R=h_{11,22}+h_{22,11}-2 h_{12,12}+O\left(h^{2}\right),
$$

and also

$$
\sqrt{g}=1+\frac{1}{2}\left(h_{11}+h_{22}\right)+O\left(h^{2}\right),
$$

which gives

$$
\sqrt{g} R^{2}=\left(h_{11,22}+h_{22,11}-2 h_{12,12}\right)^{2}+O\left(h^{3}\right) .
$$

In momentum space, each derivative $\partial_{\nu}$ produces a factor of $k_{\nu}$, and so one obtains

$$
\sqrt{g} R^{2}=h_{\mu \nu} V_{\mu \nu, \rho \sigma} h_{\rho \sigma},
$$

where $V_{\mu \nu, \rho \sigma}$ coincides with $M^{\prime}$ above (when the metric components are re-labeled according to $11 \rightarrow 1,22 \rightarrow 2,12 \rightarrow 3$ ).

One might wonder what the origin of the change of variables in Eq. (3.9) is. Given the three edges in Fig. 7, one can write for the metric at the origin

$$
g_{i j}=\left(\begin{array}{cc}
l_{1}^{2} & \frac{1}{2}\left(l_{3}^{2}-l_{1}^{2}-l_{2}^{2}\right) \\
\frac{1}{2}\left(l_{3}^{2}-l_{1}^{2}-l_{2}^{2}\right)^{2} & l_{2}^{2}
\end{array}\right) .
$$

The apparent contradiction with the earlier expression for $g_{i j}$ given in Eq. (2.10) arises from the different choice of coordinates in the triangles (compare Fig. 3 with Fig. 7). Inserting $l_{i}=l_{i}^{0}\left(1+\epsilon_{i}\right)$, with $l_{i}^{0}=1$ for the body principals $(i=1,2)$ and $l_{i}^{0}=\sqrt{2}$ for the diagonal $(i=3)$, one obtains

$$
\begin{aligned}
l_{1}^{2} & =\left(1+\epsilon_{1}\right)^{2}=1+h_{11}, \\
l_{2}^{2} & =\left(1+\epsilon_{2}\right)^{2}=1+h_{22}, \\
\frac{1}{2} l_{3}^{2} & =\left(1+\epsilon_{3}\right)^{2}=1+\frac{1}{2}\left(h_{11}+h_{22}\right)+h_{12},
\end{aligned}
$$

which can be inverted to give 


$$
\begin{aligned}
& \epsilon_{1}=\frac{1}{2} h_{11}-\frac{1}{8} h_{11}^{2}+O\left(h_{11}^{3}\right), \\
& \epsilon_{2}=\frac{1}{2} h_{22}-\frac{1}{8} h_{22}^{2}+O\left(h_{22}^{3}\right), \\
& \epsilon_{3}=\frac{1}{4}\left(h_{11}+h_{22}+2 h_{12}\right)-\frac{1}{32}\left(h_{11}+h_{22}+2 h_{12}\right)^{2}+O\left(h^{3}\right)
\end{aligned}
$$

and which was then used in Eq. (3.9). Thus the matrix $M_{\omega}$ was brought into the continuum form after performing a suitable local rotation from the local edge lengths to the local metric components.

The weak-field expansion for the purely gravitational part can be carried out to higher order, and the Feynman rules for the vertices of order $h^{3}, h^{4}, \ldots$ in the $R^{2}$-action of Eq. (2.27) can be derived. Since their expressions are rather complicated, they will not be recorded here.

\subsection{Lattice diffeomorphisms}

It is easy to determine the eigenvalues and eigenvectors of the matrix $M_{\omega}$ of Eq. (3.7). The eigenvalues of the matrix $M_{\omega}$ are given by

$$
\begin{aligned}
\lambda_{1}= & 0, \\
\lambda_{2}= & 0, \\
\lambda_{3}= & 24-9\left(\omega_{1}+\bar{\omega}_{1}+\omega_{2}+\bar{\omega}_{2}\right)+4\left(\omega_{1} \bar{\omega}_{2}+\bar{\omega}_{1} \omega_{2}\right) \\
& +\omega_{1} \omega_{2}^{2}+\omega_{1}^{2} \omega_{2}+\bar{\omega}_{1} \bar{\omega}_{2}^{2}+\bar{\omega}_{1}^{2} \bar{\omega}_{2},
\end{aligned}
$$

and there are thus two exact zero-modes in the weak-field limit. It should be emphasized that the exact zero-modes appear for arbitrary $\omega_{i}$, and not just for small momenta. We shall see later that their presence reflects an exact local continuous invariance of the gravitational action.

If one were interested in doing lattice perturbation theory, one would have to add a lattice gauge-fixing term to remove the zero-modes, such as the lattice analog of the term

$$
\frac{1}{\kappa^{2}}\left(\partial_{\mu} \sqrt{g(x)} g^{\mu \nu}\right)^{2},
$$

and add the necessary Faddeev-Popov non-local ghost determinant. A similar term would have to be included as well if one were to pick the lattice analog of the conformal gauge [36], to which we shall return later. If one is not doing perturbation theory, then of course the contribution of the zero-modes will cancel out between the numerator and denominator in the Feynman path integral representation for operator averages, and such a term should not be included, as in ordinary lattice formulations of Yang-Mills gauge theories.

The eigenvectors corresponding to the two zero-modes can be written as

$$
\left(\begin{array}{l}
\epsilon_{1}(\omega) \\
\epsilon_{2}(\omega) \\
\epsilon_{3}(\omega)
\end{array}\right)=\left(\begin{array}{cc}
1-\omega_{1} & 0 \\
0 & 1-\omega_{2} \\
\frac{1}{2}\left(1-\omega_{1} \omega_{2}\right) & \frac{1}{2}\left(1-\omega_{1} \omega_{2}\right)
\end{array}\right)\left(\begin{array}{l}
\chi_{1}(\omega) \\
\chi_{2}(\omega)
\end{array}\right),
$$




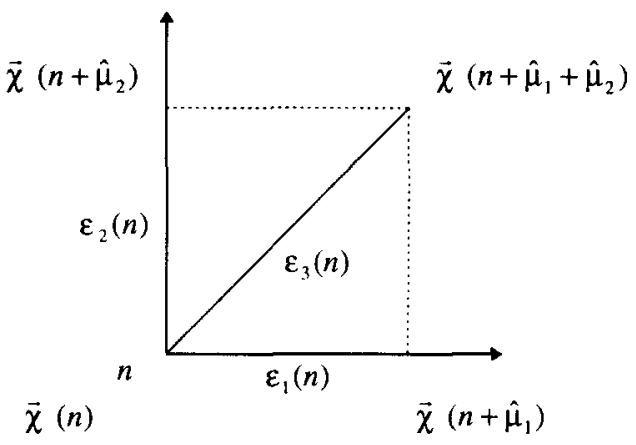

Fig. 8. Edge length gauge deformations $\epsilon_{i}(n)$, and corresponding gauge transformation vector field $\chi(n)$, defined on the sites.

where $\chi_{1}(\omega)$ and $\chi_{2}(\omega)$ are arbitrary. One might worry that the above result is restricted to two dimensions. This is not the case. Completely analogous zero-modes are found for the Regge action in three [6] and four [4] dimensions, leading to expressions rather similar to Eq. (3.21), with as expected $d$ zero-modes in $d$ dimensions. As we shall see, this is not a coincidence. We give here for comparison the corresponding expression in three dimensions [6], obtained from the weak-field expansion of the Regge action,

$$
\left(\begin{array}{l}
\epsilon_{1}(\omega) \\
\epsilon_{2}(\omega) \\
\epsilon_{4}(\omega) \\
\epsilon_{3}(\omega) \\
\epsilon_{5}(\omega) \\
\epsilon_{6}(\omega) \\
\epsilon_{7}(\omega)
\end{array}\right)=\left(\begin{array}{ccc}
1-\omega_{1} & 0 & 0 \\
0 & 1-\omega_{2} & 0 \\
0 & 0 & 1-\omega_{4} \\
\frac{1}{2}\left(1-\omega_{1} \omega_{2}\right) & \frac{1}{2}\left(1-\omega_{1} \omega_{2}\right) & 0 \\
\frac{1}{2}\left(1-\omega_{1} \omega_{4}\right) & 0 & \frac{1}{2}\left(1-\omega_{1} \omega_{4}\right) \\
0 & \frac{1}{2}\left(1-\omega_{2} \omega_{4}\right) & \frac{1}{2}\left(1-\omega_{2} \omega_{4}\right) \\
\frac{1}{3}\left(1-\omega_{1} \omega_{2} \omega_{4}\right) & \frac{1}{3}\left(1-\omega_{1} \omega_{2} \omega_{4}\right) & \frac{1}{3}\left(1-\omega_{1} \omega_{2} \omega_{4}\right)
\end{array}\right)\left(\begin{array}{c}
\chi_{1}(\omega) \\
\chi_{2}(\omega) \\
\chi_{3}(\omega)
\end{array}\right),
$$

again with $\chi_{1}(\omega), \chi_{2}(\omega)$ and $\chi_{3}(\omega)$ arbitrary gauge functions (in the binary notation for the edges, the indices $1,2,4$ correspond to the body principals, the indices $3,5,6$ to the face diagonals, and 7 to the body diagonal).

It is useful to look at the above relations, and in particular Eq. (3.21), in real space. The replacement $e^{i k_{i}} \rightarrow e^{d / d x}$ and $e^{a \cdot \nabla} f(x)=f(x+a)$ yields

$$
\begin{aligned}
& \epsilon_{1}(n)=\chi_{1}(n)-\chi_{1}\left(n+\hat{\mu}_{1}\right), \\
& \epsilon_{2}(n)=\chi_{2}(n)-\chi_{2}\left(n+\hat{\mu}_{2}\right), \\
& \epsilon_{3}(n)=\frac{1}{2} \chi_{1}(n)+\frac{1}{2} \chi_{2}(n)-\frac{1}{2} \chi_{1}\left(n+\hat{\mu}_{1}+\hat{\mu}_{2}\right)-\frac{1}{2} \chi_{2}\left(n+\hat{\mu}_{1}+\hat{\mu}_{2}\right) .
\end{aligned}
$$

For our notation, we refer to the drawing in Fig. 8. Note how the arbitrary gauge variations act on the two ends of an edge. This is true in any dimension, where elementary local gauge transformations are always defined on the vertices of the lattice. The above gauge transformation law is in fact remarkably simple for those edges that lie in the direction of the chosen coordinates, namely 


$$
\delta l_{i j}^{2}=\chi_{i}^{\prime}-\chi_{j}^{\prime},
$$

where $i$ and $j$ labels the end points of the edge, and we have rescaled the arbitrary functions $\chi$ by $l_{i j}^{2}$ so that the quantities $\chi_{i}^{\prime}$ now have dimensions of length squared.

It is easy to see that the above equations solve the constraint $\sum_{i} \epsilon_{i}(n)=0$ at the vertex $n$ where $\boldsymbol{\chi}_{n} \neq 0$,

$$
\begin{aligned}
\sum_{i=1}^{6} \epsilon_{i}(n)= & \chi_{1}(n)+\frac{1}{2} \chi_{1}(n)+\frac{1}{2} \chi_{2}(n)+\chi_{2}(n)-\chi_{1}(n) \\
& -\frac{1}{2} \chi_{1}(n)-\frac{1}{2} \chi_{2}(n)-\chi_{2}(n) \\
= & 0 .
\end{aligned}
$$

It can be written equivalently in terms of variations of the squared edge lengths meeting at the vertex $n$, labeled clockwise around the vertex $n$ starting with the edge in the positive 1 direction,

$$
a\left(\delta l_{n 1}^{2}+\delta l_{n 3}^{2}+\delta l_{n 4}^{2}+\delta l_{n 6}^{2}\right)+b\left(\delta l_{n 2}^{2}+\delta l_{n 5}^{2}\right)=0,
$$

with $a$ and $b$ arbitrary constants at this point. In other words, gauge variations of the squared edge lengths are recognized as special variations, where all edges meeting at a point (in two dimensions) are considered, and which either have the explicit form given in Eq. (3.23) for the weak-field case, or equivalently (and more generally) satisfy a set of defining constraints such as the one in Eq. (3.26).

Incidentally, it should be noted here that conformal transformations, which in the continuum take the form $\delta g_{\mu \nu}(x)=g_{\mu \nu}(x) \delta \varphi(x)$, have a natural lattice analog. They contract (or expand) locally all the edges meeting on a given vertex $n$ by the same amount,

$$
\delta l_{i}^{2}(n)=l_{i}^{2}(n) \delta \varphi(n),
$$

and therefore $d o$ change locally the curvature at $n$. When constructed in this way, they can be considered orthogonal to the $\chi$ transformations of Eqs. (3.21) and (3.23).

The cosmological constant term can also be shown to be invariant under the same set of continuous local transformations, since, using the same notation for the expansion about the square lattice, one obtains

$$
\sum_{h} A_{h}=\sum_{n}\left[1+\frac{1}{3}\left(\epsilon_{1}(n)+\epsilon_{2}(n)\right)+O\left(\epsilon^{2}\right)\right],
$$

and at the vertex $P_{n}$ where $\boldsymbol{\chi}_{n} \neq 0$ one has again

$$
\sum_{i=1}^{4} \epsilon_{i}(n)=\chi_{1}(n)+\chi_{2}(n)-\chi_{1}(n)-\chi_{2}(n)=0,
$$

where the sum is over the four edges pointing in the four principal directions. Written in terms of the variations of the squared edge lengths, one has 


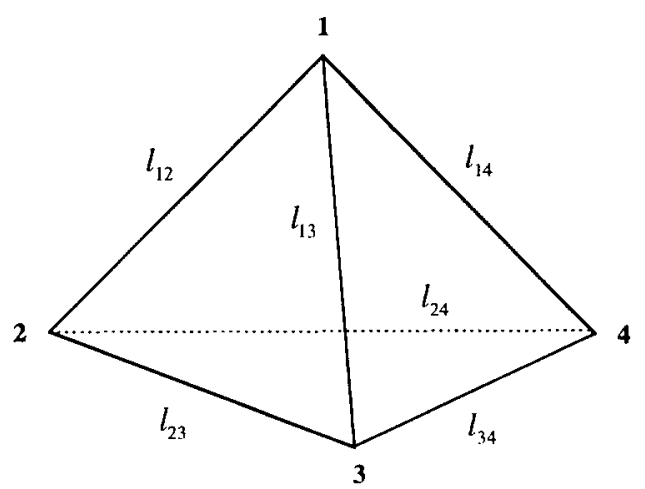

Fig. 9. Tetrahedral tessellation of the two-sphere, with arbitrary edge length assignments.

$$
\delta l_{n 1}^{2}+\delta l_{n 3}^{2}+\delta l_{n 4}^{2}+\delta l_{n 6}^{2}=0 \text {. }
$$

In general one has in two dimensions six edges meeting at a vertex, and therefore four allowed constraints on the gauge edge length variations. In conclusion we have exhibited an exact local gauge invariance of the gravitational action in the weak-field limit. Later on we shall show that it corresponds precisely to the lattice analog of the diffeomorphisms.

It is important to notice that the appearance of zero-modes in the weak-field expansion is not specific to the expansion about flat space. One can look at the same procedure for variations about spaces which are classical solutions for the gravitational action with a cosmological constant term as in Eq. (2.27), such as the regular tessellations of the two-sphere [7]. In the following we will consider edge length fluctuations about the regular tetrahedron (with 6 edges), octahedron ( 12 edges), and icosahedron (30 edges).

After expanding about the equilateral configuration, the action at the stationary point reduces to

$$
I=\lambda 8 \pi \sqrt{a / \lambda}+a 8 \pi / \sqrt{a / \lambda}=16 \pi \sqrt{a \lambda},
$$

in fact independently of the tessellation considered. Vanishing of the linear terms in the small fluctuation expansion gives for the average edge length

$$
l_{0}=\left[c \pi^{2}(4 a / \lambda)\right]^{1 / 4}
$$

with $c=16 / 3,4 / 3,16 / 75$ for the tetrahedron, octahedron and icosahedron, respectively. For fluctuations about the classical solution for a tetrahedral tessellation of $S^{2}$ (see Fig. 9) the small edge length fluctuation matrix gives rise to the following coefficients:

$$
\begin{aligned}
\epsilon_{12}^{2} & \rightarrow 16 \sqrt{a \lambda}\left(54-6 \sqrt{3} \pi+5 \pi^{2}\right) / 81 \pi, \\
\epsilon_{12} \epsilon_{13} & \rightarrow 16 \sqrt{a \lambda} \pi / 9 \\
\epsilon_{12} \epsilon_{15} & \rightarrow 64 \sqrt{a \lambda}\left(-27+3 \sqrt{3} \pi+2 \pi^{2}\right) / 81 \pi,
\end{aligned}
$$

with the remaining coefficients being determined by symmetry. The small fluctuation matrix is therefore given by 


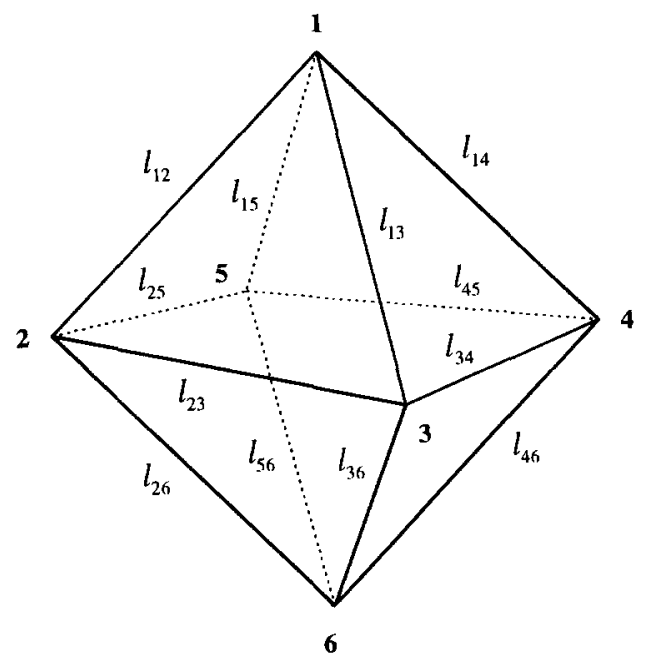

Fig. 10. Octahedral tessellation of the two-sphere, with arbitrary edge length assignments.

$$
\frac{8 \pi \sqrt{a \lambda}}{9}\left(\begin{array}{cccccc}
\mu & 1 & 1 & 1 & 1 & 2-\mu \\
1 & \mu & 1 & 2-\mu & 1 & 1 \\
1 & 1 & \mu & 1 & 2-\mu & 1 \\
1 & 2-\mu & 1 & \mu & 1 & 1 \\
1 & 1 & 2-\mu & 1 & \mu & 1 \\
2-\mu & 1 & 1 & 1 & 1 & \mu
\end{array}\right) \text {, }
$$

where $\mu=2\left(5 \pi^{2}-6 \sqrt{3} \pi+54\right) / 9 \pi^{2} \approx 1.5919$ (the $\lambda / a$ dependence has disappeared since the couplings $a$ and $\lambda$ only appear in the dimensionless combination $\sqrt{a \lambda}$ ). The eigenvalues of the above matrix (neglecting the constants in front of it) are 0 (with multiplicity 2), 2( $\mu-1$ ) (with multiplicity 3 ) and 6 (with multiplicity 1 ). The zeromodes correspond to flat directions for which deformations of the edge lengths leave the lattice geometry unchanged. The multiplicities of the eigenvalues agree with the dimensions of the irreducible representations of the symmetry group of the tetrahedron.

For the octahedron (see Fig. 10) one obtains instead the following coefficients of the small fluctuation matrix:

$$
\begin{aligned}
\epsilon_{12}^{2} & \rightarrow 2 \sqrt{a \lambda}\left(216-12 \sqrt{3} \pi+5 \pi^{2}\right) / 27 \pi, \\
\epsilon_{12} \epsilon_{13} & \rightarrow 8 \sqrt{a \lambda}\left(-27-3 \sqrt{3} \pi+2 \pi^{2}\right) / 27 \pi, \\
\epsilon_{12} \epsilon_{14} & \rightarrow 4 \sqrt{a \lambda}\left(54+\pi^{2}\right) / 9 \pi, \\
\epsilon_{12} \epsilon_{34} & \rightarrow 8 \sqrt{a \lambda}\left(-54+3 \sqrt{3} \pi+\pi^{2}\right) / 27 \pi, \\
\epsilon_{12} \epsilon_{46} & \rightarrow 4 \sqrt{a \lambda}\left(108+12 \sqrt{3} \pi+\pi^{2}\right) / 27 \pi,
\end{aligned}
$$

again with the remaining coefficients being determined by symmetry. Up to a common factor of $2 \sqrt{a \lambda} / 27 \pi$, the eigenvalues of the $12 \times 12$ small fluctuation matrix are given 


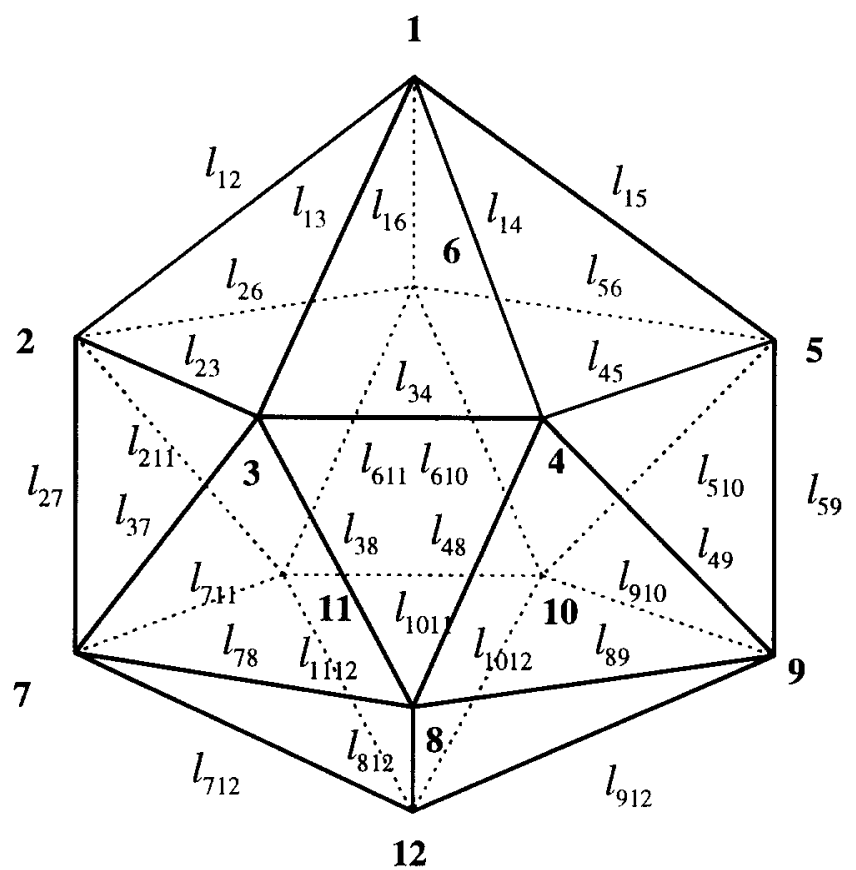

Fig. 11. Icosahedral tessellation of the two-sphere, with arbitrary edge length assignments.

by $36 \pi^{2}$ (with multiplicity 1 ), 972 (with multiplicity 2), and $8(3 \sqrt{3}-\pi)^{2}$ (with multiplicity 3), and zero (with multiplicity 6).

Finally, for the icosahedron (shown in Fig. 11) one computes the following coefficients of the small fluctuation matrix:

$$
\begin{aligned}
\epsilon_{12}^{2} & \rightarrow 16 \sqrt{a \lambda}\left(270-6 \sqrt{3} \pi+\pi^{2}\right) / 135 \pi, \\
\epsilon_{12} \epsilon_{13} & \rightarrow 16 \sqrt{a \lambda}\left(-675-30 \sqrt{3} \pi+8 \pi^{2}\right) / 675 \pi, \\
\epsilon_{12} \epsilon_{14} & \rightarrow 16 \sqrt{a \lambda}\left(270-6 \sqrt{3}+\pi^{2}\right) / 135 \pi, \\
\epsilon_{12} \epsilon_{34} & \rightarrow 32 \sqrt{a \lambda}\left(-675+15 \sqrt{3} \pi+2 \pi^{2}\right) / 675 \pi, \\
\epsilon_{12} \epsilon_{45} & \rightarrow 16 \sqrt{a \lambda}\left(-675+15 \sqrt{3} \pi+2 \pi^{2}\right) / 675 \pi, \\
\epsilon_{12} \epsilon_{38} & \rightarrow 16 \sqrt{a \lambda}\left(-675+15 \sqrt{3} \pi+2 \pi^{2}\right) / 675 \pi, \\
\epsilon_{12} \epsilon_{48} & \rightarrow 16 \sqrt{a \lambda}\left(675+30 \sqrt{3} \pi+\pi^{2}\right) / 675 \pi,
\end{aligned}
$$

with the remaining coefficients being determined by symmetry. Up to a common factor of $8 \sqrt{a \lambda} / 675 \pi$, the eigenvalues of the $30 \times 30$ small edge length fluctuation matrix are given (numerically) by 12340.173 (with multiplicity 3), 7238.984 (with multiplicity 5), $888.264=90 \pi^{2}$ (with multiplicity 1), 20.887 (with multiplicity 3 ), and zero (with multiplicity 18 ).

The presence of the zero-modes is related to the gauge (diffeomorphism) invariance of the gravitational action. The previous results can in fact be summarized as 


$$
\begin{aligned}
\text { Tetrahedron }\left(N_{0}=4\right): & 2 \text { zero-modes } \\
\text { Octahedron }\left(N_{0}=6\right): & 6 \text { zero-modes } \\
\text { Icosahedron }\left(N_{0}=12\right): & 18 \text { zero-modes }
\end{aligned}
$$

If the number of zero-modes for each triangulation of the sphere is denoted by $N_{\mathrm{z} . \mathrm{m}}$, then the results can be re-expressed as

$$
N_{\text {z.m. }}=2 N_{0}-6 \text {, }
$$

which agrees with the expectation that in the continuum limit, $N_{0} \rightarrow \infty, N_{\text {z.m. }} / N_{0}$ should approach the constant value $d$ in $d$ space-time dimensions, which is the number of local parameters for a diffeomorphism. On the lattice the diffeomorphisms correspond to local deformations of the edge lengths about a vertex, which leave the local geometry physically unchanged, the latter being described by the values of local lattice operators corresponding to local volumes, and curvatures. The lesson is that the correct count of zero-modes will in general only be recovered asymptotically for large triangulations, where $N_{0}$ is roughly much larger than the number of neighbors to a point in $d$ dimensions. It should be possible to find a similar pattern in higher dimensions.

\subsection{Edge lengths as metric components}

Returning to the weak-field expansion about flat space, it is easy to see that the above lattice gauge transformation corresponds to the diffeomorphisms in the continuum. Using the relationship between the metric perturbations and the edge length variations, obtained by choosing coordinates axes along the edges (as in Eq. (3.16) and Fig. 7),

$$
\delta g_{i j}(n)=\left(\begin{array}{cc}
\delta l_{1}^{2}(n) & \frac{1}{2}\left(\delta l_{3}^{2}(n)-\delta l_{1}^{2}(n)-\delta l_{2}^{2}(n)\right) \\
\frac{1}{2}\left(\delta l_{3}^{2}(n)-\delta l_{1}^{2}(n)-\delta l_{2}^{2}(n)\right) & \delta l_{2}^{2}(n)
\end{array}\right),
$$

one obtains from Eq. (3.23) the result

$$
\begin{aligned}
\delta g_{11} & =\delta l_{1}^{2}=2 \chi_{1}(n)-2 \chi_{1}\left(n+\hat{\mu}_{1}\right) \approx-2 \partial_{1} \chi_{1}, \\
\delta g_{22} & =\delta l_{2}^{2}=2 \chi_{2}(n)-2 \chi_{2}\left(n+\hat{\mu}_{2}\right) \approx-2 \partial_{2} \chi_{2}, \\
\delta g_{12} & =\frac{1}{2}\left(\delta l_{3}^{2}-\delta l_{1}^{2}-\delta l_{2}^{2}\right) \\
& =\chi_{1}\left(n+\hat{\mu}_{1}\right)-\chi_{1}\left(n+\hat{\mu}_{1}+\hat{\mu}_{2}\right)+\chi_{2}\left(n+\hat{\mu}_{2}\right)-\chi_{2}\left(n+\hat{\mu}_{1}+\hat{\mu}_{2}\right) \\
& \approx-\partial_{2} \chi_{1}-\partial_{1} \chi_{2},
\end{aligned}
$$

which can then be combined into the single familiar expression

$$
\delta g_{\mu \nu}=-\partial_{\mu} \chi_{\nu}-\partial_{\nu} \chi_{\mu},
$$

and which is indeed the correct gauge variation in the weak-field limit. 
Conversely, the above form of the lattice gauge transformation, Eq. (3.23), can be obtained from the form of infinitesimal diffeomorphisms in the continuum. In order to see this result, start from the definitions of diffeomorphisms

$$
g_{\mu \nu}^{\prime}\left(x^{\prime}\right)=\frac{\partial x^{\rho}}{\partial x_{\mu}^{\prime}} \frac{\partial x^{\sigma}}{\partial x_{\nu}^{\prime}} g_{\rho \sigma}(x),
$$

as transformations which leave the infinitesimal line element, as well as any other coordinate invariant quantity, unchanged

$$
d s^{2} \equiv g_{\mu \nu}^{\prime}\left(x^{\prime}\right) d x^{\prime \mu} d x^{\prime \nu}=d s^{2} \equiv g_{\rho \sigma}(x) d x^{\rho} d x^{\sigma}
$$

under an arbitrary change of coordinates

$$
x^{\prime \mu}=x^{\mu}+\chi^{\mu}(x) .
$$

For infinitesimal variations one obtains

$$
\begin{aligned}
g_{\mu \nu}^{\prime}\left(x^{\prime}\right) & =g_{\mu \nu}(x)+\delta g_{\mu \nu}(x) \\
& =g_{\mu \nu}(x)-g_{\mu \lambda}(x) \partial_{\nu} \chi^{\lambda}(x)-g_{\lambda \nu}(x) \partial_{\mu} \chi^{\lambda}(x)+O\left(\chi^{2}\right) .
\end{aligned}
$$

The above relationships express the well-known fact that metrics related by a coordinate transformation describe the same physical manifold. In the discrete case it reflects the invariance of the lattice action under local deformations of the simplicial manifold which leave the local curvatures unchanged [3]. Since the continuum metric degrees of freedom correspond on the lattice to the values of edge lengths squared, one would expect to find analogous deformations of the edge lengths that leave the lattice geometry invariant, the latter being specified by the local lattice areas and curvatures, in accordance with the principle of discussing the geometric properties of the lattice theory in terms of lattice quantities only. Clearly the distance between lattice vertices will change under such a transformation, in accordance with the fact that only distances between fixed points will remain the same. This invariance is spoiled by the presence of the triangle inequalities, which places a constraint on how far the individual edge lengths can be deformed. In the perturbative, weak-field expansion about a fixed background the triangle inequalities are not seen to any order in perturbation theory, they represent non-perturbative constraints.

These considerations are further illustrated by the following elementary example [38]. In one dimension (zero space, one time dimension) one can discretize the line by introducing $N$ points, joined by segments of lengths $l_{n}$. The only invariant term in one dimension is obviously the length of the curve,

$$
L(l)=\sum_{n=1}^{N} l_{n} .
$$

From the expression for the invariant line element, $d s^{2}=g d x^{2}$, one naturally associates $g(x)$ with $l_{n}^{2}$, and the coordinate increment with the lattice spacing, $d x=1$. One can take the view that distances can only be assigned between vertices which appear on 
some lattice in the ensemble, although this is not strictly necessary as distances can also be defined for locations that do not coincide with any specific vertex.

The above one-dimensional action has an exact local invariance (compare with Eq. (3.23) in two dimensions)

$$
\delta l_{n}=\chi_{n+1}-\chi_{n},
$$

where the $\chi_{n}$ 's represent continuous gauge transformations defined on the lattice vertices (actually, in order for the edge lengths to remain positive, one should also require $\chi_{n}-\chi_{n+1}<l_{n}$, which is satisfied for sufficiently small $\chi^{\prime}$ 's). These transformations are in fact remarkably close in structure to the ones found in two dimensions (see Eq. (3.24)). Physically, the local invariance reflects the re-parameterization, or coordinate invariance, of the original continuum action $L=\int d x \sqrt{g(x)}$. It is the discrete form of the change $\delta g(x)=2 g \partial \chi$. Variations of the edges which satisfy Eq. (3.47) leave the physical length of the curve unchanged. In addition, given any two points on the curve, independent local gauge transformations can be performed on any of the vertices situated between the two points, while at the same time maintaining the same physical distance between them (which, for any assignments of edge lengths, is simply obtained by adding up the intervening edge lengths). It justifies the name lattice diffeomorphisms for the transformations of Eq. (3.47). ${ }^{5}$

In two dimensions one starts from the relationship of Eq. (3.16) between the squared edge lengths and the metric, and uses the expression for metric perturbations given in Eq. (3.39). From Eq. (3.45) one obtains

$$
\begin{aligned}
\delta l_{1}^{2} & =\delta g_{11}=-2 l_{1}^{2} \partial_{1} \chi^{1}+\left(l_{1}^{2}+l_{2}^{2}-l_{3}^{2}\right) \partial_{1} \chi^{2}, \\
\delta l_{2}^{2} & =\delta g_{22}=-2 l_{2}^{2} \partial_{2} \chi^{2}+\left(l_{1}^{2}+l_{2}^{2}-l_{3}^{2}\right) \partial_{2} \chi^{1}, \\
\delta l_{3}^{2} & =\delta g_{11}+\delta g_{22}+2 \delta g_{12} \\
& =\left(-l_{1}^{2}+l_{2}^{2}-l_{3}^{2}\right)\left(\partial_{1} \chi^{1}+\partial_{2} \chi^{1}\right)+\left(l_{1}^{2}-l_{2}^{2}-l_{3}^{2}\right)\left(\partial_{1} \chi^{2}+\partial_{2} \chi^{2}\right) .
\end{aligned}
$$

After introducing appropriate finite differences for the fields $\chi^{\mu}$ one then has

$$
\begin{aligned}
& \delta l_{1}^{2}=-2 l_{1}^{2}\left(\chi_{1}^{1}-\chi_{0}^{1}\right)+\left(l_{1}^{2}+l_{2}^{2}-l_{3}^{2}\right)\left(\chi_{1}^{2}-\chi_{0}^{2}\right), \\
& \delta l_{2}^{2}=-2 l_{2}^{2}\left(\chi_{2}^{2}-\chi_{0}^{2}\right)+\left(l_{1}^{2}+l_{2}^{2}-l_{3}^{2}\right)\left(\chi_{2}^{1}-\chi_{0}^{1}\right), \\
& \delta l_{3}^{2}=\left(-l_{1}^{2}+l_{2}^{2}-l_{3}^{2}\right)\left(\chi_{3}^{1}-\chi_{0}^{1}\right)+\left(l_{1}^{2}-l_{2}^{2}-l_{3}^{2}\right)\left(\chi_{3}^{2}-\chi_{0}^{2}\right) .
\end{aligned}
$$

Here upper indices on $\chi$ label the components, while lower indices indicate the position. Taking $\chi_{1}=\chi_{2}=\chi_{3}=0, \chi_{0} \neq 0$, as well as $l_{1}=l_{2}=1, l_{3}=\sqrt{2}$ (as appropriate for the square lattice), one finally obtains the simple result

\footnotetext{
${ }^{5}$ There is here an (incomplete) analogy with ordinary lattice gauge theories, in the sense that if one defines $U_{n}=e^{l_{n}}$ and $V_{n}=e^{X_{n}}$, then the gauge transformation law of Eq. (3.47) can be rewritten as $U_{n} \rightarrow$ $V_{n}^{-1} U_{n} V_{n+1}$, which parallels the gauge transformation law for the $S U(N)$ gauge fields $U_{n \mu}$ in $d$ dimensions, $U_{n \mu} \rightarrow V_{n}^{-1} U_{n \mu} V_{n+\mu}$, where $V_{n \mu}$ are arbitrary $N \times N S U(N)$ matrices. The "update" $U_{n} \rightarrow V \cdot U_{n}$ corresponds here to $l_{n} \rightarrow l_{n}+\delta l_{n}$.
} 


$$
\begin{aligned}
& \delta l_{1}^{2}=2 \chi_{0}^{1}, \\
& \delta l_{2}^{2}=2 \chi_{0}^{2}, \\
& \delta l_{3}^{2}=2\left(\chi_{0}^{1}+\chi_{0}^{2}\right),
\end{aligned}
$$

which is indeed equivalent to Eq. (3.23). This shows that the zero-modes described in Eq. (3.23) correspond to lattice diffeomorphisms. (The term lattice coordinate transformations would appear to be equally suitable, provided one identifies the directions associated with the edges with a preferred coordinate system, and identifies changes in these coordinates as corresponding to variations in the squared edge lengths which leave the local curvatures unchanged.) The case of flat space is obviously the simplest. By moving the location of the vertices around in flat space, one can find a different assignment of edge lengths which represents the same flat geometry. This leads to a $d \cdot N_{0}$-parameter family of transformations for the edge lengths in flat space, and to a set of equivalent metrics which are all related by lattice diffeomorphisms, i.e. deformations of the edge lengths which leave the local curvature invariants unchanged, and respect the triangle inequalities.

In conclusion, the previous analysis shows a direct correspondence between gauge transformations on the simplicial lattice

$$
l_{i}^{2} \longrightarrow l_{i}^{2}+\delta l_{i}^{2}(\chi),
$$

and the analogous diffeomorphisms in the continuum

$$
g_{\mu \nu}(x) \longrightarrow g_{\mu \nu}(x)+\delta g_{\mu \nu}(\chi(x)) .
$$

These transformations, in which suitable deformations of the edge lengths are shown to correspond to the local gauge transformations, should be contrasted with the set of what can be called trivial coordinate transformations. For a given assignment of edge lengths, introduce an arbitrary coordinate system, and a corresponding flat metric, within each triangle (or simplex in higher dimensions). Coordinate changes can then be performed within any triangle such that

$$
\begin{aligned}
& l_{01}^{2}(n)=l_{01}^{2}(n), \\
& l_{02}^{\prime 2}(n)=l_{02}^{2}(n), \\
& l_{12}^{\prime 2}(n)=l_{12}^{2}(n)
\end{aligned}
$$

within each triangle. These diffeomorphisms are trivial, in the sense that they correspond to a change in an arbitrary coordinate system, which was not part of the theory to begin with, as Regge's lattice theory is formulated exclusively in terms of coordinateindependent squared edge lengths, and not piecewise flat continuum metrics, which are highly degenerate.

The confusion between the two types of invariance is, in our opinion, at the root of the erroneous conclusions drawn in Ref. [39], where it is argued that Regge gravity always needs a non-local gauge-fixing term, to compensate for the fact that in the 
functional integral for gravity the integration is over "invariants", the edge lengths. As the edge lengths correspond to components of the metric (see Eqs. (2.7), (3.18), (3.21), (3.23)), this cannot be true. The above discussion shows in detail that the situation is more subtle, and that a non-local additional, and in our opinion ad-hoc, gauge-fixing term will most likely lead to an incorrect weighting, as already pointed out in $[40]$.

\section{Arbitrary curved backgrounds}

The previous discussion dealt with the case of an expansion of the gravitational action about flat space, or a regular tessellation of the sphere, a manifold of constant curvature. To complete our discussion, we now turn to the more complex task of exhibiting explicitly the local gauge invariance of the simplicial theory, for an arbitrary background simplicial complex. To this end we write

$$
l_{i}^{2}=l_{0 i}^{2}+q_{i}+\delta l_{i}^{2},
$$

where $q_{i}$ describes an arbitrary but small deviation from a regular lattice, and $\delta l_{i}^{2}$ is a gauge fluctuation, whose form needs to be determined. We shall keep terms $O\left(q^{2}\right)$ and $O\left(q \delta l^{2}\right)$, but neglect terms $O\left(\delta l^{4}\right)$.

The squared volumes $V_{n}^{2}(\sigma)$ of $n$-dimensional simplices $\sigma$ are given by homogeneous polynomials of order $\left(l^{2}\right)^{n}$. In particular for the area of a triangle $A_{\Delta}$ with arbitrary edges $l_{1}, l_{2}, l_{3}$ one has

$$
\delta A_{\Delta}^{2}=\frac{1}{8}\left(-l_{1}^{2}+l_{2}^{2}+l_{3}^{2}\right) \delta l_{1}^{2}+\frac{1}{8}\left(l_{1}^{2}-l_{2}^{2}+l_{3}^{2}\right) \delta l_{2}^{2}+\frac{1}{8}\left(l_{1}^{2}+l_{2}^{2}-l_{3}^{2}\right) \delta l_{3}^{2},
$$

and similarly for the other quantities in Eqs. (2.16)-(2.18) which are needed in order to construct the action.

For our notation in two dimensions we will refer to Fig. 12. The subsequent figures 13 and 14 illustrate the difference between a gauge deformation of the surface which leaves the area and curvature at the point labeled by 0 invariant, and a physical deformation which corresponds to a re-assignment of edge lengths meeting at the vertex 0 such that it alters the area and curvature at 0 . In the following we will characterize unambiguously what we mean by the two different operations.

\subsection{Equilateral lattices}

First consider the expansion about a deformed equilateral lattice, for which $l_{0 i}=1$ to start with. A motivation for this choice is provided by the fact that in the numerical studies of two-dimensional gravity the averages of the squared edge lengths in the three principal directions turn out to be equal, $\left\langle l_{1}^{2}\right\rangle=\left\langle l_{2}^{2}\right\rangle=\left\langle l_{3}^{2}\right\rangle$. The baricentric area associated with vertex 0 is then given by 


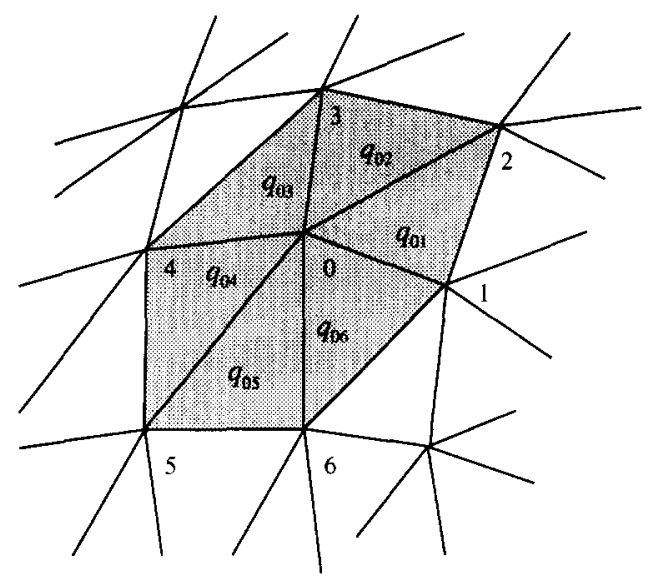

Fig. 12. Notation for an arbitrary simplicial lattice, where the edge lengths meeting at the vertex 0 have been deformed away from a regular lattice by a small amount $q_{i}$ (minimally deformed equilateral lattice).

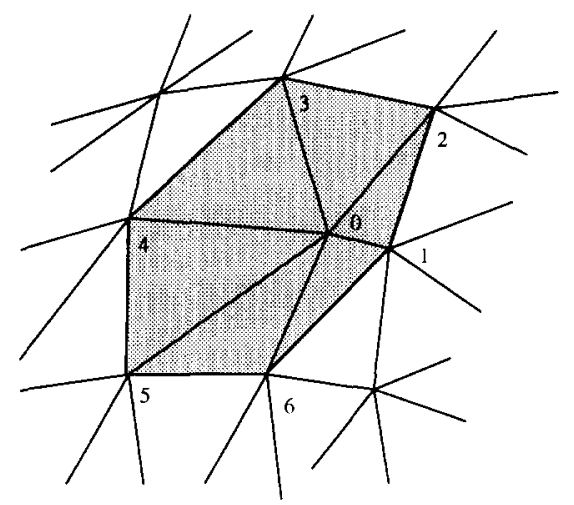

Fig. 13. Local gauge deformations of the lattice act on the edge lengths meeting at the vertex 0 , and are performed in such a way that the area and curvature at the vertex 0 are left unchanged.

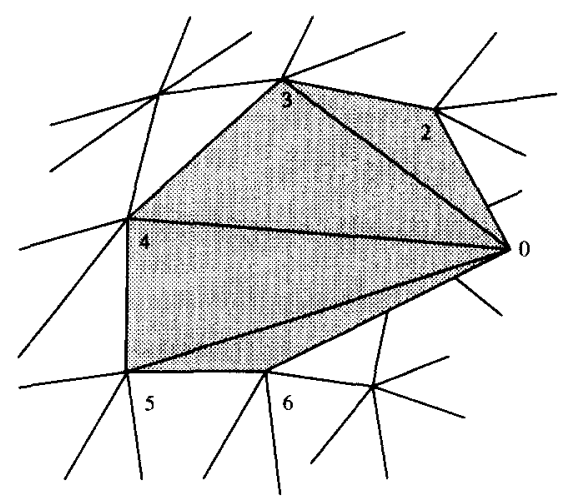

Fig. 14. Physical deformations change the area and curvature at the vertex 0 , thus changing the lattice geometry. 


$$
\begin{aligned}
A= & A_{0}(q)+\frac{1}{2 \cdot 3^{5 / 2}}\left[\delta l_{01}^{2}\left(3+q_{06}-4 q_{01}+q_{02}+q_{16}+q_{12}\right)\right. \\
& +\delta l_{02}^{2}\left(3+q_{01}-4 q_{02}+q_{03}+q_{12}+q_{23}\right) \\
& +\delta l_{03}^{2}\left(3+q_{02}-4 q_{03}+q_{04}+q_{23}+q_{34}\right) \\
& +\delta l_{04}^{2}\left(3+q_{03}-4 q_{04}+q_{05}+q_{34}+q_{45}\right) \\
& +\delta l_{05}^{2}\left(3+q_{04}-4 q_{05}+q_{06}+q_{45}+q_{56}\right) \\
& \left.+\delta l_{06}^{2}\left(3+q_{05}-4 q_{06}+q_{01}+q_{56}+q_{16}\right)\right]+O\left(\delta l^{4}\right) .
\end{aligned}
$$

Our normalization is such that $A_{0}=\frac{\sqrt{3}}{2}$ for $q_{i}=0$. Equivalently one can write, in more compact notation, at the vertex 0

$$
A=A_{0}(q)+\frac{1}{3} v_{A}(q) \cdot \delta l^{2}+O\left(\delta l^{4}\right),
$$

with $\delta l^{2}=\left(\delta l_{01}^{2}, \ldots, \delta l_{06}^{2}\right)$. After adding the contributions from the neighboring vertices one obtains

$$
\sum_{P_{0} \ldots P_{6}} A=\sum_{P_{0} \ldots P_{6}} A_{0}(q)+v_{A}(q) \cdot \delta l^{2}+O\left(\delta l^{4}\right) .
$$

Therefore the area associated with the vertex 0 will remain unchanged provided the variations in the squared edge lengths meeting at 0 satisfy the constraint

$$
v_{A}(q) \cdot \delta l^{2}=0
$$

This is nothing but the curved space equivalent of the condition of Eq. (3.30), which for the flat equilateral lattice takes the form

$$
\sum_{i=1}^{6} \delta l_{i}^{2}(n)=0
$$

Alternatively, if one considers a dual subdivision, one has to consider the dual area associated with vertex 0 . In this case one has

$$
\begin{aligned}
A= & A_{0}(q)+\frac{1}{4 \cdot 3^{3 / 2}}\left[\delta l_{01}^{2}\left(1+2 q_{06}-4 q_{01}+2 q_{02}\right)\right. \\
& +\delta l_{02}^{2}\left(1+2 q_{01}-4 q_{02}+2 q_{03}\right)+\delta l_{03}^{2}\left(1+2 q_{02}-4 q_{03}+2 q_{04}\right) \\
& +\delta l_{04}^{2}\left(1+2 q_{03}-4 q_{04}+2 q_{05}\right)+\delta l_{05}^{2}\left(1+2 q_{04}-4 q_{05}+2 q_{06}\right) \\
& \left.+\delta l_{06}^{2}\left(1+2 q_{05}-4 q_{06}+2 q_{01}\right)\right]+O\left(\delta l^{4}\right) .
\end{aligned}
$$

leading to a result formally similar (in fact in this case identical) to the baricentric case.

A similar calculation can be done for the curvature associated with vertex 0 . One has for the deficit angle at 0

$$
\begin{aligned}
\delta= & \delta_{0}(q)+\frac{1}{3^{3 / 2}}\left[\delta l_{01}^{2}\left(3-2 q_{06}-q_{01}-2 q_{02}+q_{16}+q_{12}\right)\right. \\
& \left.+\delta l_{02}^{2}\left(3-2 q_{01}-q_{02}-2 q_{03}+q_{12}+q_{23}\right)+\ldots\right]+O\left(\delta l^{4}\right) .
\end{aligned}
$$


and therefore for the variation of the sum of the deficit angles surrounding 0

$$
\Delta\left(\sum_{h} \delta_{h}\right)=\sum_{P_{0} \ldots P_{6}} \Delta \delta
$$

and

$$
\sum_{P_{0} \ldots P_{6}} \delta=\sum_{P_{0} \ldots P_{6}} \delta_{0}(q)+v_{R}(q) \cdot \delta l^{2}+O\left(\delta l^{4}\right),
$$

with in this case, as expected, $v_{R}(q) \equiv 0$.

Finally for the curvature squared associated with vertex 0 one computes

$$
\begin{aligned}
\delta^{2} / A= & \delta_{0}^{2} / A_{0}(q)+\frac{4}{3^{3 / 2}}\left[\delta l _ { 0 1 } ^ { 2 } \left(q_{01}+q_{02}+q_{03}+q_{04}+q_{05}+q_{06}-q_{12}-q_{23}\right.\right. \\
& \left.-q_{34}-q_{45}-q_{56}-q_{16}\right)+\delta l_{02}^{2}\left(q_{01}+q_{02}+q_{03}+q_{04}+q_{05}+q_{06}\right. \\
& \left.\left.-q_{12}-q_{23}-q_{34}-q_{45}-q_{56}-q_{16}\right)+\ldots\right]+O\left(\delta l^{4}\right)
\end{aligned}
$$

Adding up all seven contributions one gets

$$
\Delta\left(\sum_{h} \delta_{h}^{2} / A_{h}\right)=\sum_{P_{0} \ldots P_{6}} \Delta\left(\delta^{2} / A\right),
$$

and therefore

$$
\sum_{P_{0} \ldots P_{6}} \delta^{2} / A=\sum_{P_{0} \ldots P_{6}}\left(\delta^{2} / A\right)_{0}+v_{R^{2}}(q) \cdot \delta l^{2}+O\left(\delta l^{4}\right) .
$$

In this case the curvature squared associated with the vertex 0 will remain unchanged, provided the variations in the squared edge lengths meeting at 0 satisfy the constraint

$$
v_{R^{2}}(q) \cdot \delta l^{2}=0
$$

which provides a second constraint on the edge length variations $\delta l^{2}$ at the vertex 0 . Again this constraint is the generalization to curved space of the condition of Eq. (3.26), which was valid for flat space.

\subsection{Square lattice}

A similar calculation can be performed for the square lattice with $l_{01}=l_{02}=1$ and $l_{03}=\sqrt{2}$. The baricentric area associated with vertex 0 is now given by

$$
\begin{aligned}
A= & A_{0}(q)+\frac{1}{24}\left[\delta l_{01}^{2}\left(4-4 q_{01}+q_{02}+q_{16}\right)+\delta l_{02}^{2}\left(q_{01}-2 q_{02}+q_{03}+q_{12}+q_{23}\right)\right. \\
& +\delta l_{03}^{2}\left(4+q_{02}-4 q_{03}+q_{34}\right)+\delta l_{04}^{2}\left(4-4 q_{04}+q_{05}+q_{34}\right) \\
& \left.+\delta l_{05}^{2}\left(q_{04}-2 q_{05}+q_{06}+q_{45}+q_{56}\right)+\delta l_{06}^{2}\left(4+q_{05}-4 q_{06}+q_{16}\right)\right] \\
& +O\left(\delta l^{4}\right) .
\end{aligned}
$$


Our normalization here is such that $A_{0}=1$ for $q_{i}=0$. Summing up all relevant contributions, one can write

$$
\sum_{P_{0} \ldots P_{6}} A=\sum_{P_{0} \ldots P_{6}} A_{0}(q)+v_{A}^{\prime}(q) \cdot \delta l^{2}+O\left(\delta l^{4}\right),
$$

leading to the invariance constraint on the edge length variations

$$
v_{A}^{\prime}(q) \cdot \delta l^{2}=0
$$

This provides a first constraint on the edge length variations $\delta l^{2}$ at the vertex 0 , for the deformed square lattice.

For the dual (Voronoi) area associated with vertex 0 one has instead

$$
\begin{aligned}
A= & A_{0}(q)+\frac{1}{16}\left[\delta l_{01}^{2}\left(2-3 q_{01}+2 q_{02}+2 q_{06}-q_{16}-q_{12}\right)\right. \\
& +\delta l_{02}^{2}\left(-2+2 q_{01}-3 q_{02}+2 q_{03}+q_{12}+q_{23}\right) \\
& +\delta l_{03}^{2}\left(2+2 q_{02}-3 q_{03}+2 q_{04}-q_{23}-q_{34}\right) \\
& +\delta l_{04}^{2}\left(2+2 q_{03}-3 q_{04}+2 q_{05}-q_{34}-q_{45}\right) \\
& +\delta l_{05}^{2}\left(-2+2 q_{04}-3 q_{05}+2 q_{06}+q_{45}+q_{56}\right) \\
& \left.+\delta l_{06}^{2}\left(2+2 q_{01}+2 q_{05}-3 q_{06}-q_{56}-q_{16}\right)\right]+O\left(\delta l^{4}\right),
\end{aligned}
$$

leading to a constraint similar to the one for the baricentric area. For the curvature associated with vertex 0 one has

$$
\begin{aligned}
\delta= & \delta_{0}(q)+\frac{1}{4}\left[\delta l_{01}^{2}\left(2-2 q_{06}-q_{01}-q_{02}+q_{16}+q_{12}\right)+\delta l_{02}^{2}\left(2-q_{01}-q_{03}\right)\right. \\
& +\delta l_{03}^{2}\left(2-q_{02}-q_{03}-2 q_{04}+q_{23}+q_{34}\right) \\
& +\delta l_{04}^{2}\left(2-2 q_{03}-q_{04}-q_{05}+q_{34}+q_{45}\right)+\delta l_{05}^{2}\left(2-q_{04}-q_{06}\right) \\
& \left.+\delta l_{06}^{2}\left(2-q_{05}-q_{06}-2 q_{01}+q_{56}+q_{16}\right)\right]+O\left(\delta l^{4}\right)
\end{aligned}
$$

which gives now

$$
\sum_{P_{0} \ldots P_{6}} \delta=\sum_{P_{0} \ldots P_{6}} \delta_{0}(q)+v_{R}^{\prime}(q) \cdot \delta l^{2}+O\left(\delta l^{4}\right),
$$

with $v_{R}^{\prime}(q) \equiv 0$.

Finally, the curvature squared associated with just the vertex 0 is given by

$$
\begin{aligned}
\delta^{2} / A= & \delta_{0}^{2} / A_{0}(q)+\frac{1}{2}\left[\delta l _ { 0 1 } ^ { 2 } \left(q_{01}+q_{02}+q_{03}+q_{04}+q_{05}+q_{06}-q_{12}-q_{23}\right.\right. \\
& \left.-q_{34}-q_{45}-q_{56}-q_{16}\right)+\delta l_{02}^{2}\left(q_{01}+q_{02}+q_{03}+q_{04}+q_{05}+q_{06}\right. \\
& \left.\left.-q_{12}-q_{23}-q_{34}-q_{45}-q_{56}-q_{16}\right)+\ldots\right]+O\left(\delta l^{4}\right) .
\end{aligned}
$$

The labeling in the previous formulae is a bit clumsy in dealing with nearest next-nearest neighbor interactions; for the labeling in the following formula we refer to Fig. 15. 


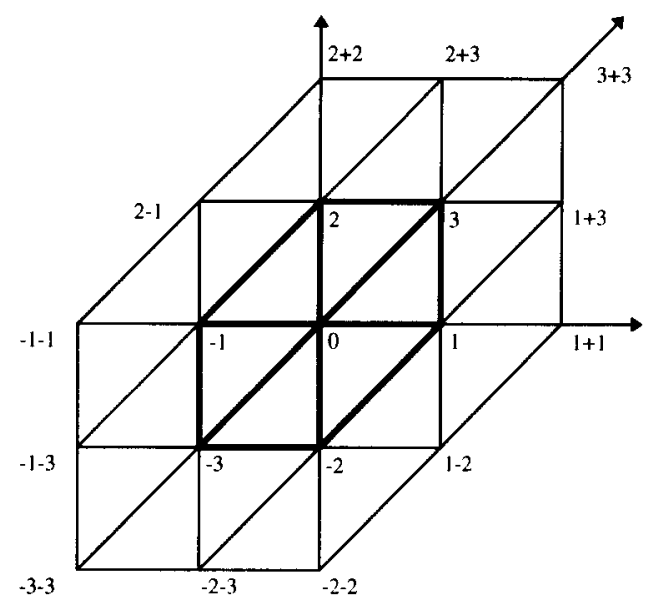

Fig. 15. Labeling of lattice vertices for the expansion of the curvature-squared term around a minimally deformed square lattice.

Adding up the contributions from the seven distinct vertices $0-6$ one obtains for the term linear in $\delta l^{2}$

$$
\begin{aligned}
\frac{1}{2} & {\left[\delta l _ { 0 , 1 } ^ { 2 } \left(4 q_{0,1}+2 q_{0,2}+q_{0,-1}-q_{0,-2}-q_{0,3}+2 q_{0,-3}-q_{1,3}-2 q_{2,3}-q_{-1,-3}\right.\right.} \\
& -2 q_{-2,-3}-q_{-1,2}-q_{1,-2}+q_{1,1+1}+2 q_{1,1-2}+2 q_{1,1+3}-q_{1+1,1-2} \\
& -q_{1+1,1+3}+q_{2,2+3}-2 q_{-2,1-2}-q_{-2,-2-2}-q_{-2,-2-3}+q_{-2-2,-2+1} \\
& \left.+q_{-2-2,-2-3}-2 q_{3,1+3}-q_{3,2+3}-q_{3,3+3}-q_{-3,-2-3}+q_{3+3,1+3}+q_{3+3,2+3}\right) \\
& +\delta l_{0,3}^{2}\left(-q_{0,1}-q_{0,2}+4 q_{0,3}+2 q_{0,-1}+2 q_{0,-2}+q_{0,-3}-q_{1,3}-q_{2,3}-q_{-1,-3}\right. \\
& -q_{-2,-3}-2 q_{-1,2}-2 q_{1,-2}-q_{1,1+1}-q_{1,1-2}-2 q_{1,1+3}+q_{-1,2-1}+q_{1+1,1-2} \\
& +q_{1+1,1+3}-q_{2,2+2}-q_{2,2-1}-2 q_{2,2+3}+q_{-2,1-2}+q_{2+2,2-1}+q_{2+2,2+3} \\
& \left.\left.+2 q_{3,1+3}+2 q_{3,2+3}+q_{3,3+3}-q_{3+3,1+3}-q_{3+3,2+3}\right)+\ldots\right]+O\left(\delta l^{4}\right) .
\end{aligned}
$$

which can be written in short form as

$$
\sum_{P_{0} \ldots P_{6}} \delta^{2} / A(q)=\sum_{P_{0} \ldots P_{6}}\left(\delta^{2} / A\right)_{0}+v_{R^{2}}^{\prime}(q) \cdot \delta l^{2}+O\left(\delta l^{4}\right) .
$$

Again the curvature squared associated with the vertex 0 will remain unchanged provided the variations in the squared edge lengths meeting at 0 satisfy the constraint

$$
v_{R^{2}}^{\prime}(q) \cdot \delta l^{2}=0
$$

which provides the second constraint on the edge length variations $\delta l^{2}$ at the vertex 0 , for the deformed square lattice (compare with Eq. (3.26)).

In conclusion, we have shown explicitly how gauge variations of the edge lengths at each vertex can be defined by requiring that the action contributions be locally invariant. We have looked at small deformations, but large deformations can be treated as well 


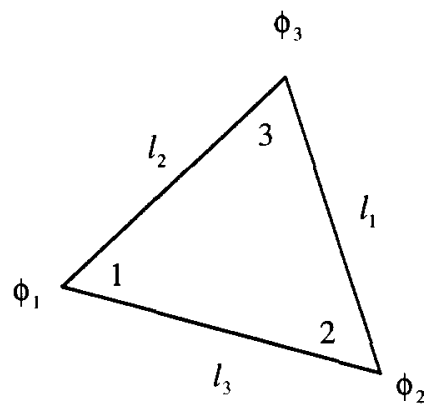

Fig. 16. Labeling of edges and fields for the construction of the scalar field action.

along the same lines, provided one is careful not to violate the triangle inequalities, which impose a non-perturbative cutoff in orbit space The same approach can also be extended to higher dimensions, leading to similar (but rather more complicated, when written out explicitly!) results. The main conclusions do not change.

\section{Scalar field}

In the previous section we have discussed the invariance properties of the lattice action for pure gravity. Next a scalar field is introduced, as the simplest type of dynamical matter that can be coupled to gravity. The scalar lattice action in the continuum is

$$
I[g, \phi]=\frac{1}{2} \int d^{2} x \sqrt{g}\left[g^{\mu \nu} \partial_{\mu} \phi \partial_{\nu} \phi+\left(m^{2}+\xi R\right) \phi^{2}\right] .
$$

The dimensionless coupling $\xi$ is arbitrary; two special cases are the minimal $(\xi=0)$ and the conformal $\left(\xi=\frac{1}{6}\right)$ coupling case. In the following we will mostly consider the case $\xi=0$.

\subsection{Construction of the lattice action}

On the lattice consider a scalar $\phi_{i}$ and define this field at the vertices of the simplices. The corresponding lattice action can be obtained through the usual procedure which replaces the original continuum metric with the induced metric on the lattice, written in terms of the edge lengths $[4,6]$. Here we shall consider only the two-dimensional case; the generalization to higher dimensions is straightforward. It is convenient to use the notation of Fig. 16, which will bring out more readily the symmetries of the resulting lattice action. Here coordinates will be picked in each triangle along the $(1,2)$ and $(1,3)$ directions.

To construct the scalar lattice action, one performs in two dimensions the replacement

$$
g_{\mu \nu}(x) \longrightarrow g_{i j}(\Delta)=\left(\begin{array}{cc}
l_{3}^{2} & \frac{1}{2}\left(-l_{1}^{2}+l_{2}^{2}+l_{3}^{2}\right) \\
\frac{1}{2}\left(-l_{1}^{2}+l_{2}^{2}+l_{3}^{2}\right) & l_{2}^{2}
\end{array}\right),
$$


which then gives

$$
\operatorname{det} g_{\mu \nu}(x) \longrightarrow \operatorname{det} g_{i j}(\Delta)=\frac{1}{4}\left\{2\left(l_{1}^{2} l_{2}^{2}+l_{2}^{2} l_{3}^{2}+l_{3}^{2} l_{1}^{2}\right)-l_{1}^{4}-l_{2}^{4}-l_{3}^{4}\right\} \equiv 4 A_{\Delta}^{2},
$$

and also

$$
g^{\mu \nu}(x) \longrightarrow g^{i j}(\Delta)=\frac{1}{\operatorname{det} g(\Delta)}\left(\begin{array}{cc}
l_{2}^{2} & \frac{1}{2}\left(l_{1}^{2}-l_{2}^{2}-l_{3}^{2}\right) \\
\frac{1}{2}\left(l_{1}^{2}-l_{2}^{2}-l_{3}^{2}\right) & l_{3}^{2}
\end{array}\right) .
$$

For the scalar field derivatives one writes $[41,42]$

$$
\partial_{\mu} \phi \partial_{\nu} \phi \longrightarrow \Delta_{i} \phi \Delta_{j} \phi=\left(\begin{array}{cc}
\left(\phi_{2}-\phi_{1}\right)^{2} & \left(\phi_{2}-\phi_{1}\right)\left(\phi_{3}-\phi_{1}\right) \\
\left(\phi_{2}-\phi_{1}\right)\left(\phi_{3}-\phi_{1}\right) & \left(\phi_{3}-\phi_{1}\right)^{2}
\end{array}\right)
$$

which corresponds to introducing finite lattice differences defined in the usual way by

$$
\partial_{\mu} \phi \longrightarrow\left(\Delta_{\mu} \phi\right)_{i}=\phi_{i+\mu}-\phi_{i}
$$

Here the index $\mu$ labels the possible directions in which one can move from a point in a given triangle. The discrete scalar field action then takes the form

$$
\begin{aligned}
I_{m}\left(l^{2}, \phi\right)= & \frac{1}{16} \sum_{\Delta} \frac{1}{A_{\Delta}}\left[l_{1}^{2}\left(\phi_{2}-\phi_{1}\right)\left(\phi_{3}-\phi_{1}\right)+l_{2}^{2}\left(\phi_{3}-\phi_{2}\right)\left(\phi_{1}-\phi_{2}\right)\right. \\
& \left.+l_{3}^{2}\left(\phi_{1}-\phi_{3}\right)\left(\phi_{2}-\phi_{3}\right)\right] .
\end{aligned}
$$

Using the identity

$$
\left(\phi_{i}-\phi_{j}\right)\left(\phi_{i}-\phi_{k}\right)=\frac{1}{2}\left[\left(\phi_{i}-\phi_{j}\right)^{2}+\left(\phi_{i}-\phi_{k}\right)^{2}-\left(\phi_{j}-\phi_{k}\right)^{2}\right],
$$

one obtains after some re-arrangements the simpler expression [41]

$$
I_{m}\left(l^{2}, \phi\right)=\frac{1}{2} \sum_{\langle i j\rangle} A_{i j}\left(\frac{\phi_{i}-\phi_{j}}{l_{i j}}\right)^{2}
$$

where $A_{i j}$ is the dual (Voronoi) area associated with the edge $i j$. In terms of the edge length $l_{i j}$ and the dual edge length $h_{i j}$, connecting neighboring vertices in the dual lattice, one has $A_{i j}=\frac{1}{2} h_{i j} l_{i j}$ (see Fig. 17). Other choices for the lattice subdivision will lead to a similar formula for the lattice action, but with the Voronoi dual volumes replaced by their appropriate counterparts in the new lattice.

For the edge of length $l_{1}$ the dihedral dual volume contribution is given by

$$
A_{l_{1}}=\frac{l_{1}^{2}\left(l_{2}^{2}+l_{3}^{2}-l_{1}^{2}\right)}{16 A_{123}}+\frac{l_{1}^{2}\left(l_{4}^{2}+l_{5}^{2}-l_{1}^{2}\right)}{16 A_{234}}=\frac{1}{2} l_{1} h_{1},
$$

with $h_{1}$ the length of the edge dual to $l_{1}$. The baricentric dihedral volume for the same edge would be simply 


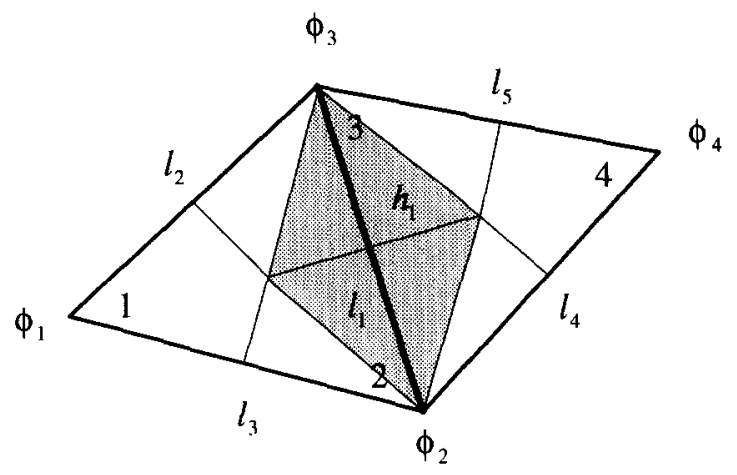

Fig. 17. Dual area associated with the edge $\ell_{1}$ (shaded area), and the corresponding dual link $h_{1}$.

$$
A_{l_{1}}=\left(A_{123}+A_{234}\right) / 3 \text {. }
$$

It is well known that one of the disadvantages of the Voronoi construction is the lack of positivity of the dual volumes, as already pointed out in [7]. Thus some of the weights appearing in Eq. (5.9) can be negative for such an action. On the other hand, for the baricentric subdivision this problem does not arise, as the areas $A_{i j}$ are always positive due to the enforcement of the triangle inequalities. It is immediate to generalize the action of Eq. (5.9) to higher dimensions, with the two-dimensional Voronoi volumes replaced by their higher-dimensional analogs.

Mass and curvature terms can be added to the action, so that the total scalar action contribution becomes

$$
I_{m}\left(l^{2}, \phi\right)=\frac{1}{2} \sum_{\langle i j\rangle} A_{i j}\left(\frac{\phi_{i}-\phi_{j}}{l_{i j}}\right)^{2}+\frac{1}{2} \sum_{i} A_{i}\left(m^{2}+\xi R_{i}\right) \phi_{i}^{2} .
$$

The term containing the discrete analog of the scalar curvature involves the quantity

$$
A_{i} R_{i} \equiv \sum_{h \supset i} \delta_{h} \sim \sqrt{g} R
$$

In the above expression for the scalar action, $A_{i j}$ is the area associated with the edge $l_{i j}$, while $A_{i}$ is associated with the site $i$. Again there is more than one way to define the volume element $A_{i}$ [7], but under reasonable assumptions, such as positivity, one expects to get equivalent results in the lattice continuum limit. In the following we shall mainly consider the simplest form for the scalar action, with $m^{2}=\xi=0$.

One of the simplest problems which can be studied analytically in the continuum as well as on the lattice is the analysis of small fluctuations about some classical background solution. In the continuum, the weak-field expansion is often performed by expanding the metric and the action about flat Euclidean space

$$
g_{\mu \nu}=\delta_{\mu \nu}+\kappa h_{\mu \nu}
$$

In four dimensions $\kappa=\sqrt{32 \pi G}$, which shows that the weak-field expansion there corresponds to an expansion in powers of $G$. In two dimensions this is no longer 
the case and the relation between $\kappa$ and $G$ is lost; instead one should regard $\kappa$ as a dimensionless expansion parameter which is eventually set to one, $\kappa=1$, at the end of the calculation. The procedure will be sensible as long as wildly fluctuating geometries are not important in two dimensions (on the lattice or in the continuum). The influence of the latter configurations can only be studied by numerical simulations of the full path integral $[11,30]$.

In the continuum, the Feynman rules are obtained by expanding out the action in the weak fields $h_{\mu \nu}(x)$,

$$
\begin{aligned}
\frac{1}{2} \int d^{2} x \sqrt{g} g^{\mu \nu} \partial_{\mu} \phi \partial_{\nu} \phi= & \frac{1}{2} \int d^{2} x(\partial \phi)^{2}+\frac{1}{2} \int d^{2} x h_{\mu \nu}\left\{\frac{1}{2} \delta_{\mu \nu}(\partial \phi)^{2}-\partial_{\mu} \phi \partial_{\nu} \phi\right\} \\
& +O\left(h^{2}\right),
\end{aligned}
$$

and by then transforming the resulting expressions to momentum space.

On the lattice the action is again expanded in the small fluctuation fields $\epsilon_{i}$, which depend on the specific choice of parameterization for the flat background lattice - a convenient starting point is (in two dimensions) the square lattice with diagonals. It is convenient to define the edge variables at the midpoints of the links [18]. For the edge lengths one then defines the lattice Fourier transforms as

$$
\begin{aligned}
& \epsilon_{1}(n)=\int_{-\pi}^{\pi} \int_{-\pi}^{\pi} \frac{d^{2} k}{(2 \pi)^{2}} e^{-i k \cdot n-i k_{1} / 2} \epsilon_{1}(k), \\
& \epsilon_{2}(n)=\int_{-\pi}^{\pi} \int_{-\pi}^{\pi} \frac{d^{2} k}{(2 \pi)^{2}} e^{-i k \cdot n-i k_{2} / 2} \epsilon_{2}(k), \\
& \epsilon_{3}(n)=\int_{-\pi}^{\pi} \int_{-\pi}^{\pi} \frac{d^{2} k}{(2 \pi)^{2}} e^{-i k \cdot n-i k_{1} / 2-i k_{2} / 2} \epsilon_{3}(k),
\end{aligned}
$$

while the scalar fields are still defined on the vertices, and are Fourier transformed in the usual way, namely

$$
\phi(n)=\int_{-\pi}^{\pi} \int_{-\pi}^{\pi} \frac{d^{2} p}{(2 \pi)^{2}} e^{-i p \cdot n} \phi(p) .
$$

These formulae are completely analogous to the ones used in developing the perturbative expansion for lattice gauge theories [44]. They are easy to generalize to higher dimensions when a simplicial subdivision of a hypercubic lattice is employed, as first suggested in [4]. Following this procedure, the Feynman rules for the lattice scalar field action were derived in [18] (using the Voronoi dual volumes), and shown to agree completely with the continuum Feynman rules for small momenta. For more details, and the computation of the Feynman diagrams relevant to the conformal anomaly, we refer the interested reader to the cited work. 


\subsection{Equilateral lattice}

In order to compare and analyze the difference between the two volume discretizations (baricentric vs. dual) for the scalar action, it will be useful to look at their form for small deformations of the edges about a regular lattice, such as an equilateral or a square one. One would expect that the precise form of the discretization should not matter, as long as the correct long distance (small momentum) properties are preserved. Let us see how this can come about.

The next step is therefore the expansion of the lattice scalar field action of Eq. (5.9) with volumes $A_{i j}$ defined via for example a baricentric subdivision, starting from an equilateral lattice with $l_{i}^{2}=l_{i}^{02}+\delta l_{i}^{2}$, and $l_{i}^{0}=1$. It will be sufficient to limit oneself to the contributions coming from one site $(0)$ and its six neighbors (1-6), which for the kinetic term is given by

$$
\begin{aligned}
& \left(\phi_{1}-\phi_{0}\right)^{2}\left[\frac{1}{4 \sqrt{3}}+\frac{1}{24 \sqrt{3}}\left(\delta l_{06}^{2}-4 \delta l_{01}^{2}+\delta l_{02}^{2}+\delta l_{16}^{2}+\delta l_{12}^{2}\right)+O\left(\delta l^{4}\right)\right] \\
& +\left(\phi_{2}-\phi_{0}\right)^{2}\left[\frac{1}{4 \sqrt{3}}+\frac{1}{24 \sqrt{3}}\left(\delta l_{01}^{2}-4 \delta l_{02}^{2}+\delta l_{03}^{2}+\delta l_{12}^{2}+\delta l_{23}^{2}\right)+O\left(\delta l^{4}\right)\right] \\
& +\left(\phi_{3}-\phi_{0}\right)^{2}\left[\frac{1}{4 \sqrt{3}}+\frac{1}{24 \sqrt{3}}\left(\delta l_{02}^{2}-4 \delta l_{03}^{2}+\delta l_{04}^{2}+\delta l_{23}^{2}+\delta l_{34}^{2}\right)+O\left(\delta l^{4}\right)\right] \\
& +\left(\phi_{4}-\phi_{0}\right)^{2}\left[\frac{1}{4 \sqrt{3}}+\frac{1}{24 \sqrt{3}}\left(\delta l_{03}^{2}-4 \delta l_{04}^{2}+\delta l_{05}^{2}+\delta l_{34}^{2}+\delta l_{45}^{2}\right)+O\left(\delta l^{4}\right)\right] \\
& +\left(\phi_{5}-\phi_{0}\right)^{2}\left[\frac{1}{4 \sqrt{3}}+\frac{1}{24 \sqrt{3}}\left(\delta l_{04}^{2}-4 \delta l_{05}^{2}+\delta l_{06}^{2}+\delta l_{45}^{2}+\delta l_{56}^{2}\right)+O\left(\delta l^{4}\right)\right] \\
& +\left(\phi_{6}-\phi_{0}\right)^{2}\left[\frac{1}{4 \sqrt{3}}+\frac{1}{24 \sqrt{3}}\left(\delta l_{05}^{2}-4 \delta l_{06}^{2}+\delta l_{01}^{2}+\delta l_{56}^{2}+\delta l_{16}^{2}\right)+O\left(\delta l^{4}\right)\right]
\end{aligned}
$$

For our notation and labeling of the edge lengths we refer again to Fig. 12. In the case of the dual (Voronoi) subdivision, the results is identical to this order except for the replacement of the coefficient $\frac{1}{24 \sqrt{3}} \rightarrow \frac{1}{12 \sqrt{3}}$, which can be interpreted as a rescaling of the gravitational coupling between the metric field and the scalar. For fields that are smoothly varying on the scale of the cutoff, the scalar action contribution is invariant under a gauge transformation on the edges acting at the origin 0 , if the defining condition for gauge transformations, analogous to Eq. (3.26) pertaining to the square lattice in the weak-field expansion, is satisfied

$$
\sum_{i=1}^{6} \delta l_{i}^{2}(n) \approx 0 .
$$

This result is also very similar to what happens in one dimensions, where the exact invariance of the scalar action can be written down explicitly with more ease [38].

For the same (equilateral) lattice let us consider next the mass term; it is given by 


$$
\frac{1}{2} m^{2} \int d^{2} x \sqrt{g} \phi^{2} \sim \frac{1}{2} m^{2} \sum_{i} A_{i} \phi_{i}^{2} .
$$

Again expanding about the equilateral lattice with $l_{i}^{2}=l_{i}^{02}+\delta l_{i}^{2}$ one obtains for the baricentric subdivision

$$
\frac{1}{2} m^{2} \phi_{0}^{2}\left[\frac{\sqrt{3}}{2}+\frac{1}{12 \sqrt{3}}\left(2 \delta l_{01}^{2}+2 \delta l_{02}^{2}+\ldots+\delta l_{12}^{2}+\delta l_{23}^{2}+\ldots\right)+O\left(\delta l^{4}\right)\right],
$$

while in the dual case one has

$$
\frac{1}{2} m^{2} \phi_{0}^{2}\left[\frac{\sqrt{3}}{2}+\frac{1}{12 \sqrt{3}}\left(\delta l_{01}^{2}+\delta l_{02}^{2}+\ldots+2 \delta l_{12}^{2}+2 \delta l_{23}^{2}+\ldots\right)+O\left(\delta l^{4}\right)\right] .
$$

Again to this order the mass term is invariant under a gauge transformation on the edges acting at the origin 0 , if the defining condition for gauge transformations (compare to Eq. (3.30) obtained in the weak-field expansion) is satisfied

$$
\sum_{i=1}^{6} \delta l_{i}^{2}(n)=0 .
$$

Finally one can consider the curvature term,

$$
\frac{1}{2} \xi \int d^{2} x \sqrt{g} R \phi^{2} \sim \frac{1}{2} \xi \sum_{i} A_{i}\left(2 \delta_{i} / A_{i}\right) \phi_{i}^{2} .
$$

Its expansion is given by

$$
\frac{1}{2} \xi \phi_{0}^{2}\left[\frac{2}{\sqrt{3}}\left(\delta l_{01}^{2}+\delta l_{02}^{2}+\ldots-\delta l_{12}^{2}-\delta l_{23}^{2}-\ldots\right)+O\left(\delta l^{4}\right)\right],
$$

with baricentric and dual forms identical to all orders in $\delta l^{2}$, since $A_{i}$ does not appear in this term. Again it is obvious that this term is invariant under gauge transformations at 0 .

Further higher order terms involving the curvature, such as

$$
\int d^{2} x \sqrt{g} R g^{\mu \nu} \partial_{\mu} \phi \partial_{\nu} \phi \sim \sum_{i} 2 \frac{\delta_{i}}{A_{i}} \sum_{j \supset i} \frac{A_{i j}}{l_{i j}^{2}}\left(\phi_{i}-\phi_{j}\right)^{2},
$$

become increasingly complicated in their structure, as they involve neighbors which are further apart. They are strongly suppressed for smooth manifolds due to the presence of the deficit angle. In the baricentric case one finds

$$
\begin{aligned}
& \left(\phi_{1}-\phi_{0}\right)^{2} \frac{1}{3 \sqrt{3}}\left(2 \delta l_{01}^{2}+0 \cdot \delta l_{02}^{2}+\delta l_{03}^{2}+\delta l_{04}^{2}+\delta l_{05}^{2}+0 \cdot \delta l_{06}^{2}+\ldots\right. \\
& \left.-0 \cdot \delta l_{12}^{2}-\delta l_{23}^{2}-\delta l_{34}^{2}-\delta l_{45}^{2}-0 \cdot \delta l_{16}^{2}+\ldots\right)+O\left(\delta l^{4}\right),
\end{aligned}
$$


and we have omitted the terms involving $\left(\phi_{2}-\phi_{0}\right)^{2}$ etc. since they can be obtained by symmetry. Again for slowly varying scalar fields the above term is invariant under gauge transformations at the origin.

\subsection{Square lattice}

A similar calculation can be performed for the square background lattice with again $l_{i}^{2}=l_{i}^{02}+\delta l_{i}^{2}$, and $l_{01}=l_{02}=1$ and $l_{03}=\sqrt{2}$. Using the baricentric subdivision, the kinetic term gives

$$
\begin{aligned}
& \left(\phi_{1}-\phi_{0}\right)^{2}\left[\frac{1}{6}+\frac{1}{24}\left(\delta l_{06}^{2}-2 \delta l_{01}^{2}+\delta l_{12}^{2}\right)+O\left(\delta l^{4}\right)\right] \\
& +\left(\phi_{2}-\phi_{0}\right)^{2}\left[\frac{1}{12}+\frac{1}{48}\left(\delta l_{01}^{2}-2 \delta l_{02}^{2}+\delta l_{03}^{2}+\delta l_{12}^{2}+\delta l_{23}^{2}\right)+O\left(\delta l^{4}\right)\right] \\
& \quad+\left(\phi_{3}-\phi_{0}\right)^{2}\left[\frac{1}{6}+\frac{1}{24}\left(-2 \delta l_{03}^{2}+\delta l_{04}^{2}+\delta l_{23}^{2}\right)+O\left(\delta l^{4}\right)\right] \\
& \quad+\left(\phi_{4}-\phi_{0}\right)^{2}\left[\frac{1}{6}+\frac{1}{24}\left(\delta l_{03}^{2}-2 \delta l_{04}^{2}+\delta l_{45}^{2}\right)+O\left(\delta l^{4}\right)\right] \\
& \quad+\left(\phi_{5}-\phi_{0}\right)^{2}\left[\frac{1}{12}+\frac{1}{48}\left(\delta l_{04}^{2}-2 \delta l_{05}^{2}+\delta l_{06}^{2}+\delta l_{45}^{2}+\delta l_{56}^{2}\right)+O\left(\delta l^{4}\right)\right] \\
& \quad+\left(\phi_{6}-\phi_{0}\right)^{2}\left[\frac{1}{6}+\frac{1}{24}\left(-2 \delta l_{06}^{2}+\delta l_{01}^{2}+\delta l_{56}^{2}\right)+O\left(\delta l^{4}\right)\right] .
\end{aligned}
$$

Again for smoothly varying scalar fields, the above action will be invariant under gauge variations of the edge lengths at the vertex 0 , provided one has $\delta l_{01}^{2}+2 \delta l_{02}^{2}+\delta l_{03}^{2}+$ $\delta l_{04}^{2}+2 \delta l_{05}^{2}+\delta l_{06}^{2}=0$ (which is the scalar field action analog of Eq. (4.18)).

In the case of the dual subdivision, one obtains for the same lattice

$$
\begin{aligned}
& \left(\phi_{1}-\phi_{0}\right)^{2}\left[\frac{1}{4}+\frac{1}{16}\left(-4 \delta l_{01}^{2}+\delta l_{02}^{2}+\delta l_{16}^{2}\right)+O\left(\delta l^{4}\right)\right] \\
& \quad+\left(\phi_{2}-\phi_{0}\right)^{2}\left[0+\frac{1}{16}\left(\delta l_{01}^{2}-2 \delta l_{02}^{2}+\delta l_{03}^{2}+\delta l_{12}^{2}+\delta l_{23}^{2}\right)+O\left(\delta l^{4}\right)\right] \\
& \quad+\left(\phi_{3}-\phi_{0}\right)^{2}\left[\frac{1}{4}+\frac{1}{16}\left(-4 \delta l_{03}^{2}+\delta l_{02}^{2}+\delta l_{34}^{2}\right)+O\left(\delta l^{4}\right)\right] \\
& \quad+\left(\phi_{4}-\phi_{0}\right)^{2}\left[\frac{1}{4}+\frac{1}{16}\left(-4 \delta l_{04}^{2}+\delta l_{05}^{2}+\delta l_{34}^{2}\right)+O\left(\delta l^{4}\right)\right] \\
& \quad+\left(\phi_{5}-\phi_{0}\right)^{2}\left[0+\frac{1}{16}\left(\delta l_{04}^{2}-2 \delta l_{05}^{2}+\delta l_{06}^{2}+\delta l_{45}^{2}+\delta l_{56}^{2}\right)+O\left(\delta l^{4}\right)\right] \\
& \quad+\left(\phi_{6}-\phi_{0}\right)^{2}\left[\frac{1}{4}+\frac{1}{16}\left(-4 \delta l_{06}^{2}+\delta l_{05}^{2}+\delta l_{16}^{2}\right)+O\left(\delta l^{4}\right)\right]
\end{aligned}
$$

Due to the asymmetry of the lattice in this case, the difference between the two discretizations is quite marked. We will argue below that the dual volume form represents in fact an "improved" discretization over the baricentric form.

For the mass term one proceeds in the same way, and for the baricentric subdivision one obtains 
$\frac{1}{2} m^{2} \phi_{0}^{2}\left[1+\frac{1}{12}\left(2 \delta l_{01}^{2}+0 \cdot \delta l_{02}^{2}+2 \delta l_{03}^{2}+\ldots+\delta l_{12}^{2}+\delta l_{23}^{2}+0 \cdot l_{34}^{2}+\ldots\right)+O\left(\delta l^{4}\right)\right]$,

Again, this term is invariant under a gauge transformation if Eq. (4.18) is satisfied. For the dual (Voronoi) case one finds similarly

$$
\frac{1}{2} m^{2} \phi_{0}^{2}\left[1+\frac{1}{8}\left(\delta l_{01}^{2}-\delta l_{02}^{2}+\delta l_{03}^{2}+\ldots+\delta l_{12}^{2}+\delta l_{23}^{2}+\delta l_{34}^{2}+\ldots\right)+O\left(\delta l^{4}\right)\right] .
$$

which is invariant again if the area at 0 is invariant.

Finally for the curvature term of Eq. (5.24) one has

$$
\begin{aligned}
& \frac{1}{2} \xi \phi_{0}^{2}\left[\delta l_{01}^{2}+\delta l_{02}^{2}+\delta l_{03}^{2}+\delta l_{04}^{2}+\delta l_{05}^{2}+\delta l_{06}^{2}\right. \\
& \left.\quad-\delta l_{12}^{2}-\delta l_{23}^{2}-\delta l_{34}^{2}-\delta l_{45}^{2}-\delta l_{56}^{2}-\delta l_{16}^{2}+O\left(\delta l^{4}\right)\right] \\
& \quad+\frac{1}{2} \xi \phi_{1}^{2}\left[\delta l_{01}^{2}-\delta l_{02}^{2}-\delta l_{06}^{2}+\delta l_{12}^{2}+\delta l_{16}^{2}+O\left(\delta l^{4}\right)\right]+\ldots,
\end{aligned}
$$

with the baricentric discretization equal to the dual discretization to all orders in $\delta l^{2}$, since the local area $A_{i}$ does not appear. Again it is obvious that this term is invariant under gauge transformations at 0 .

Let us now return to the apparent discrepancy between the scalar kinetic term in the baricentric and dual discretizations. It is useful to look at the two discretized forms in momentum space. Thus we assume that the edge length fluctuations $\epsilon_{i} \equiv \delta l_{i}^{2} / 2 l_{i}^{02}$ and $\phi$ at the point $i, j$ steps in one coordinate direction and $k$ steps in the other coordinate direction from the origin, are related to the corresponding $\epsilon_{i}$ and $\phi$ at the origin by

$$
\epsilon_{i}^{(j+k)}=\omega_{1}^{j} \omega_{2}^{k} \epsilon_{i}^{(0)},
$$

where $\omega_{i}=e^{-i k_{i}}$, and $k_{i}$ is the momentum in the direction $i$. Similarly for $\phi$ one writes

$$
\phi^{(j+k)}=\omega_{1}^{\prime j} \omega_{2}^{\prime k} \phi_{i}^{(0)},
$$

where $\omega^{\prime}{ }_{i}=e^{-i p_{i}}$.

After making the substitution $\epsilon_{i} \rightarrow h_{\mu \nu}$ of Eq. (3.18) (which is the same as the change of variables in Eq. (3.9)) one obtains for the interaction in momentum space, in the dual case

$$
\begin{aligned}
& p_{1}^{2} \phi^{2}\left[\frac{1}{2}+\frac{1}{4}\left(-h_{11}+h_{22}\right)+O\left(h^{2}\right)\right] \\
& \quad+p_{2}^{2} \phi^{2}\left[\frac{1}{2}+\frac{1}{4}\left(+h_{11}-h_{22}\right)+O\left(h^{2}\right)\right] \\
& \quad+p_{1} p_{2} \phi^{2}\left[-h_{12}+O\left(h^{2}\right)\right],
\end{aligned}
$$

which indeed coincides with the weak-field expansion of the kinetic term for the original scalar field action in the continuum, Eqs. (5.1) and (5.15). In the baricentric case one obtains instead 


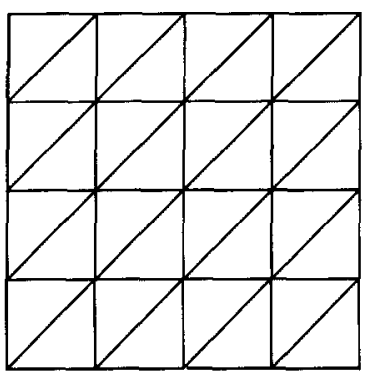

(a)

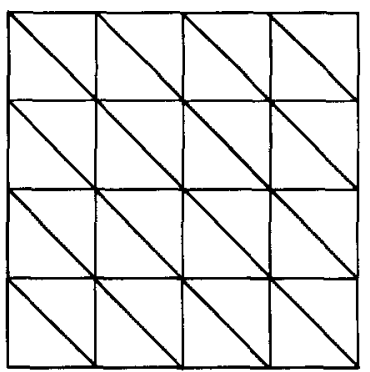

(b)

Fig. 18. Two equivalent triangulation of flat space, based on different subdivisions of the square lattice.

$$
\begin{aligned}
& p_{1}^{2} \phi^{2}\left[\frac{1}{2}+\frac{1}{6}\left(-h_{11}+h_{22}-h_{12}\right)+O\left(h^{2}\right)\right] \\
& \quad+p_{2}^{2} \phi^{2}\left[\frac{1}{2}+\frac{1}{6}\left(+h_{11}-h_{22}-h_{12}\right)+O\left(h^{2}\right)\right] \\
& +p_{1} p_{2} \phi^{2}\left[\frac{1}{3}-\frac{1}{3} h_{12}+O\left(h^{2}\right)\right],
\end{aligned}
$$

which at first looks quite different from the dual (Voronoi) volume case.

On the other hand, this result is not too surprising, as an analogous situation occurs in flat space. Consider for example the two lattices of Fig. 18 (up to now we only considered the one corresponding to 18a), and compute in each case the action for one momentum mode, which is just the inverse scalar propagator in momentum space. In the absence of any interaction terms along the lattice diagonals one has

$$
2 \sum_{\mu}\left[1-\cos p_{\mu}\right] \sim p_{1}^{2}+p_{2}^{2}+O\left(p^{4}\right) .
$$

The interaction terms along the lattice diagonals in Fig. 18a contribute

$$
2\left[1-\cos \left(p_{1}+p_{2}\right)\right] \sim p_{1}^{2}+p_{2}^{2}+2 p_{1} p_{2}+O\left(p^{4}\right),
$$

while the interaction terms along the lattice diagonals in Fig. 18b contribute

$$
2\left[1-\cos \left(p_{1}-p_{2}\right)\right] \sim p_{1}^{2}+p_{2}^{2}-2 p_{1} p_{2}+O\left(p^{4}\right) .
$$

Thus when one averages over the two equivalent contributions (since the lattice is dynamical, and both contributions are equally probable) one obtains

$$
2\left[1-\cos p_{1} \cos p_{2}\right] \sim p_{1}^{2}+p_{2}^{2}+O\left(p^{4}\right),
$$

which is now rotationally invariant to order $p^{2}$. When the same procedure is applied to the lattice scalar action in the presence of the gravitational field, Eqs. (5.35) and (5.36) can be shown to become equivalent, after a rescaling of the gravitational coupling. Still, the action based on dual Voronoi volumes of Eq. (5.35) appears to lead to a more attractive discretization, as the unwanted terms do not appear at all for the choice of lattice of Fig. 18a, and no averaging over the gravitational field fluctuations is necessary to exhibit the correct correspondence with the continuum action. How seriously one 
takes this class of problems depends on how seriously one trusts low order perturbation theory about a fixed flat background as a tool for the study of fluctuating geometries.

\subsection{Invariance properties of the scalar action}

It is instructive to look at the invariance properties of the scalar action under the continuous lattice gauge transformations defined in Eqs. (3.23) and (3.49). Physically, these local gauge transformations, which act on the vertices, correspond to re-assignments of edge lengths which leave the distance between two fixed points unchanged. In the simplest case, only two neighboring edge lengths are changed, leaving the total distance between the end points unchanged. On physical grounds one would like to maintain such an invariance also in the case of coupling to matter, just as is done in the continuum.

The scalar nature of the field requires that in the continuum under a change of coordinates $x \rightarrow x^{\prime}$,

$$
\phi^{\prime}\left(x^{\prime}\right)=\phi(x),
$$

where $x$ and $x^{\prime}$ refer to the same physical point in the two coordinate systems. Let us first look at the one-dimensional case, which is the simplest. On the lattice, as discussed previously, gauge transformations move the points around, and at the same vertex labeled by $n$ we expect

$$
\phi_{n} \rightarrow \phi_{n}^{\prime} \approx \phi_{n}+\left(\frac{\phi_{n+1}-\phi_{n}}{l_{n}}\right) \epsilon_{n}
$$

One can determine the exact form of the change needed in $\phi_{n}$ by requiring that the local variation of the scalar field action

$$
\begin{gathered}
\frac{1}{l_{n-1}+\epsilon_{n}}\left(\phi_{n}+\Delta \phi_{n}-\phi_{n-1}\right)^{2}+\frac{1}{l_{n}-\epsilon_{n}}\left(\phi_{n+1}-\phi_{n}-\Delta \phi_{n}\right)^{2} \\
-\frac{1}{l_{n-1}}\left(\phi_{n}-\phi_{n-1}\right)^{2}-\frac{1}{l_{n}}\left(\phi_{n+1}-\phi_{n}\right)^{2}=0
\end{gathered}
$$

be identically zero. Solving the resulting quadratic equation for $\Delta \phi_{n}$ one obtains a rather unwieldy expression, which to lowest order is given by [38]

$$
\begin{aligned}
\Delta \phi_{n}= & \frac{\epsilon_{n}}{2}\left[\frac{\phi_{n}-\phi_{n-1}}{l_{n-1}}+\frac{\phi_{n+1}-\phi_{n}}{l_{n}}\right] \\
& +\frac{\epsilon_{n}^{2}}{8}\left[-\frac{\phi_{n}-\phi_{n-1}}{l_{n-1}^{2}}+\frac{\phi_{n+1}-\phi_{n}}{l_{n}^{2}}+\frac{\phi_{n+1}-2 \phi_{n}+\phi_{n-1}}{l_{n-1} l_{n}}\right]+O\left(\epsilon_{n}^{3}\right),
\end{aligned}
$$

and which is indeed of the expected form (as well as being symmetric in the vertices $n-1$ and $n+1$ ). For fields which are reasonably smooth, this correction is suppressed if $\left|\phi_{n+1}-\phi_{n}\right| / l_{n} \ll 1$. On the other hand it should be clear that the functional measure $d \phi_{n}$ is no longer manifestly invariant, due to the rather involved transformation property of the scalar field. 
A similar line of argument can be pursued in higher dimensions. Thus in two dimensions one should require that locally the variation of the action contribution be again zero for edge length deformations $\delta l_{i j}$, associated with edges meeting at the vertex $i$, and which correspond to lattice gauge transformations (in the weak field, for example, they have the explicit form given in Eqs. (3.21) to (3.23)), namely

$$
\sum_{j} A_{i j}\left(l_{i j}^{2}+\delta l_{i j}^{2}\right)\left(\frac{\phi_{i}+\Delta \phi_{i}-\phi_{j}}{\sqrt{l_{i j}^{2}+\delta l_{i j}^{2}}}\right)^{2}-\sum_{j} A_{i j}\left(l_{i j}^{2}\right)\left(\frac{\phi_{i}-\phi_{j}}{\sqrt{l_{i j}^{2}}}\right)^{2}=0
$$

where $j$ labels the neighbors of the site $i$. The transformation law for $\phi_{i}$ is then determined by solving the above equation for $\Delta \phi_{i}$, given an arbitrary gauge variation $\delta l^{2}(\chi)$ at the vertex $i$.

\subsection{Equations of motion and lattice energy-momentum tensor}

The equations of motion for $\phi_{i}$ are obtained from

$$
\frac{\partial}{\partial \phi_{k}}\left\{\sum_{\langle i j\rangle} A_{i j}\left(\frac{\phi_{i}-\phi_{j}}{l_{i j}}\right)^{2}+m^{2} \sum_{i} A_{i} \phi_{i}^{2}\right\}=0,
$$

and read

$$
\sum_{j(i)} \frac{A_{i j}}{l_{i j}} \cdot \frac{\phi_{i}-\phi_{j}}{l_{i j}}+m^{2} A_{i} \phi_{i}=0,
$$

where the notation $j(i)$ indicates that the site $j$ is taken to be adjacent to $i$. For the choice of indices in Fig. 12 (see also Fig. 17), the equation with $i=0$ reads

$$
-\frac{2}{A_{01}+\ldots+A_{06}}\left\{\frac{A_{01}}{l_{01}} \cdot \frac{\phi_{1}-\phi_{0}}{l_{01}}+\ldots+\frac{A_{06}}{l_{06}} \cdot \frac{\phi_{6}-\phi_{0}}{l_{06}}\right\}+m^{2} \phi_{0}=0,
$$

and represents the discrete analog of $\frac{1}{\sqrt{g}} \partial_{\mu} g^{\mu \nu} g \partial_{\nu} \phi+m^{2} \phi=0$. Eq. (5.47) can be rewritten, for $m^{2}=0$, as

$$
\sum_{j(i)}\left(\frac{A_{i j}}{A_{i} d}\right) \frac{\phi_{i}-\phi_{j}}{l_{i j}^{2}} \equiv \sum_{j(i)} P_{i j} \frac{\phi_{i}-\phi_{j}}{l_{i j}^{2}}=0 .
$$

The weights $P_{i j}=A_{i j} / A_{i} d$ can be interpreted as normalized hopping amplitudes, with a normalization condition

$$
\sum_{j(i)} P_{i j}=1
$$

It is easy to verify that this last property does not depend on the way the original lattice has been subdivided in order to construct the dual lattice. Both the Voronoi construction 
of Eq. (2.17) and the baricentric one of Eq. (2.18) lead to the same normalization for $P_{i j}$. And indeed it is easy to show that this result holds in higher dimensions as well.

Some very interesting properties regarding the spectrum of the Laplacian on flat random lattices have been worked out in [24,19] and the reader is referred to these papers for further details. Perhaps one of the most interesting results which can be derived by the method of replicas in the weak disorder limit is that, at least in sufficiently low dimension, the spectrum of the Laplacian coincides with the continuum result at low frequencies, with subleading corrections which then reflect specific aspects of the edge length distribution such as its Poissonian form [24,38]. In other words, quenched random lattices and regular lattices give the same (continuum) result at low frequencies.

In the continuum the energy-momentum tensor for matter described by action $I_{m}$ is defined via the relationship

$$
\delta I_{m}=\frac{1}{2} \int d^{d} x \sqrt{g} T^{\mu \nu} \delta g_{\mu \nu} .
$$

For infinitesimal gauge variations, which have the form

$$
\begin{aligned}
\delta g_{\mu \nu}(x) & =-g_{\mu \lambda}(x) \partial_{\nu} \chi^{\lambda}(x)-g_{\lambda \nu}(x) \partial_{\mu} \chi^{\lambda}(x)-\partial_{\lambda} g_{\mu \nu}(x) \chi^{\lambda}(x) \\
& \equiv \Delta_{\chi} g_{\mu \nu},
\end{aligned}
$$

one expects $\delta I_{m}=0$. After an integration by parts in Eq. (5.51), one then obtains

$$
\left(T_{\lambda}^{\mu}\right)_{; \mu}=0
$$

The energy-momentum tensor defined by Eq. (5.51) will be conserved if and only if the matter action is a scalar. More concretely, for one real scalar field of mass $m$, one has

$$
T_{\mu \nu}=\partial_{\mu} \phi \partial_{\nu} \phi-\frac{1}{2} g_{\mu \nu}\left(\partial_{\lambda} \phi \partial^{\lambda} \phi+m^{2} \phi^{2}\right) .
$$

Taking the trace one obtains

$$
T_{\mu}^{\mu}=\left(\frac{2-d}{2}\right) \partial_{\mu} \phi \partial^{\mu} \phi-\frac{d}{2} m^{2} \phi^{2} .
$$

It is natural to proceed on the lattice in analogy with the continuum case. For the scalar action contribution of Eq. (5.9) one computes the variation

$$
\delta I_{m}=\sum_{k} \sum_{i j}\left(\phi_{i}-\phi_{j}\right)^{2} \frac{\partial}{\partial l_{k}^{2}}\left(\frac{A_{i j}}{2 l_{i j}^{2}}\right) \delta l_{k}^{2} .
$$

Since the derivative term inside the sum is non-zero only for edges $i j$ which belong to triangles touching the edge $k$ (see Fig. 19), only four terms contribute to the sum over $\langle i j\rangle$. It is actually more convenient to start from the equivalent form for the scalar action given in Eq. (5.7), and one obtains 


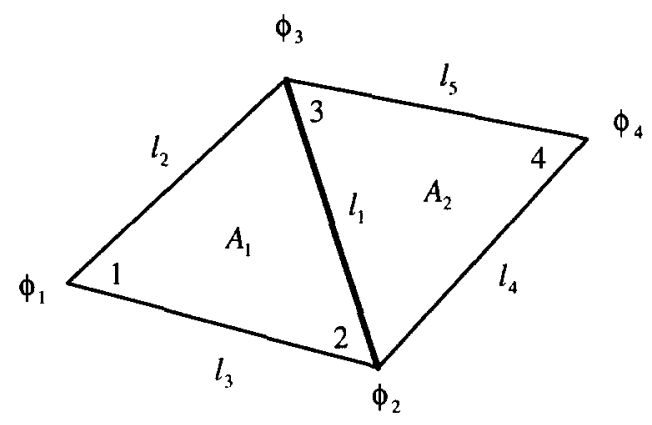

Fig. 19. Notation for Eq. (5.57) describing the variation of the action.

$$
\begin{aligned}
\delta I_{m}= & \frac{1}{16}\left[\frac{1}{16 A_{1}^{3}}\left\{l_{1}^{2}\left(l_{2}^{2}+l_{3}^{2}\right)-\left(l_{2}^{2}-l_{3}^{2}\right)^{2}\right\}\left(\phi_{2}-\phi_{1}\right)\left(\phi_{3}-\phi_{1}\right)\right. \\
& \left.+\frac{1}{16 A_{2}^{3}}\left\{l_{1}^{2}\left(l_{4}^{2}+l_{5}^{2}\right)-\left(l_{4}^{2}-l_{5}^{2}\right)^{2}\right\}\left(\phi_{2}-\phi_{4}\right)\left(\phi_{3}-\phi_{4}\right)\right] \delta l_{1}^{2} \\
& +\frac{1}{16}[\ldots] \delta l_{2}^{2}+\ldots
\end{aligned}
$$

Using the definition for the dual volumes (see Eq. (2.17), and Fig. 20 for our notation here) one can rewrite the above expression more compactly as

$$
\begin{aligned}
\delta I_{m}= & \frac{1}{8}\left[\frac{A_{11}}{A_{1}^{2}}\left(\phi_{2}-\phi_{1}\right)\left(\phi_{3}-\phi_{1}\right)+\frac{A_{24}}{A_{2}^{2}}\left(\phi_{2}-\phi_{4}\right)\left(\phi_{3}-\phi_{4}\right)\right] \delta l_{1}^{2} \\
& +\frac{1}{8}[\ldots] \delta l_{2}^{2}+\ldots
\end{aligned}
$$

Therefore one can introduce the quantities $T_{k}$ such that

$$
\delta I_{m}=\frac{1}{2} \sum_{k} T_{k}\left(l^{2}\right) \delta l_{k}^{2},
$$

with

$$
T_{1}=\frac{1}{4}\left\{\frac{A_{11}}{A_{1}^{2}}\left(\phi_{2}-\phi_{1}\right)\left(\phi_{3}-\phi_{1}\right)+\frac{A_{24}}{A_{2}^{2}}\left(\phi_{2}-\phi_{4}\right)\left(\phi_{3}-\phi_{4}\right)\right\},
$$

associated with the edge labeled by 1 in Fig. 19, and similarly for all the other edges in the lattice. It is clear that this equation defines the analog of the energy-momentum tensor in the discrete case.

The coefficients in the above expansion in $\delta l_{i}^{2}$ can be regarded as the components of the Regge lattice analog of the energy-momentum tensor,

$$
T_{k}=T_{\mu \nu} l_{k}^{\mu} l_{k}^{\nu}
$$

just as we can define for the simplicial components of the metric tensor [45]

$$
g_{k}=g_{\mu \nu} l_{k}^{\mu} l_{k}^{\nu} \equiv l_{i}^{2}
$$

From Eqs. (2.7) and (3.39) 


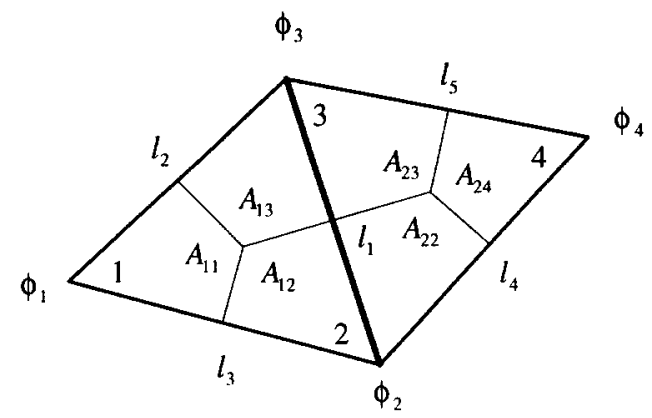

Fig. 20. Labeling of the dual areas appearing in Eq. (5.58).

$$
\delta g_{i j}\left(l^{2}\right)=\frac{1}{2}\left[\delta l_{0 i}^{2}+\delta l_{0 j}^{2}-\delta l_{i j}^{2}\right],
$$

and therefore within triangle 1 (with vertices $1,2,3$ and choosing coordinates along 23 and 21), one has (see Fig. 20)

$$
\delta l_{1}^{2}=\delta g_{11}, \quad \delta l_{2}^{2}=\delta g_{22}, \quad \delta l_{3}^{2}=\delta g_{11}+\delta g_{22}-2 \delta g_{12} .
$$

For one triangle (for example, triangle 1 in Fig. 19) one obtains

$$
T^{\mu \nu} \delta g_{\mu \nu} \longrightarrow\left(T^{11}+T^{12}\right) \delta l_{1}^{2}+\left(T^{22}+T^{12}\right) \delta l_{2}^{2}-T^{12} \delta l_{3}^{2} .
$$

In the lattice case it is clear that, inserting for the variation of the squared edge lengths corresponding to gauge variations, as in Eqs. (3.21) and (3.23), and then equating the resulting coefficients of the arbitrary gauge parameters $\chi_{n}^{i}$ to zero, gives the discrete equation of conservation for the energy-momentum tensor.

On the other hand, the equations of motion for $l_{i}^{2}$ (as opposed to the equations of motion for $\phi$, which are given in Eq. (5.48)) are obtained directly from Eq. (5.58), namely

$$
\begin{aligned}
\frac{\partial I_{m}\left[l^{2}\right]}{\partial l_{i}^{2}}= & \frac{A_{11}}{16 A_{1}^{2}}\left[\left(\phi_{2}-\phi_{1}\right)^{2}+\left(\phi_{3}-\phi_{1}\right)^{2}-\left(\phi_{3}-\phi_{2}\right)^{2}\right] \\
& +\frac{A_{24}}{16 A_{2}^{2}}\left[\left(\phi_{2}-\phi_{4}\right)^{2}+\left(\phi_{3}-\phi_{4}\right)^{2}-\left(\phi_{3}-\phi_{2}\right)^{2}\right]=0 .
\end{aligned}
$$

It corresponds to the continuum equation $T_{\mu \nu}=0$. In the presence of a cosmological constant term, $\lambda \sum_{t} A_{t}$, an additional term appears in the equations of motion, which become, again with the notation of Figs. 19 and 20,

$$
\begin{aligned}
& \frac{A_{11}}{16 A_{1}^{2}}\left[\left(\phi_{2}-\phi_{1}\right)^{2}+\left(\phi_{3}-\phi_{1}\right)^{2}-\left(\phi_{3}-\phi_{2}\right)^{2}\right] \\
& \quad+\frac{A_{24}}{16 A_{2}^{2}}\left[\left(\phi_{2}-\phi_{4}\right)^{2}+\left(\phi_{3}-\phi_{4}\right)^{2}-\left(\phi_{3}-\phi_{2}\right)^{2}\right] \\
& \quad+\lambda\left[\frac{1}{16 A_{1}}\left(l_{2}^{2}+l_{3}^{2}-l_{1}^{2}\right)+\frac{1}{16 A_{2}}\left(l_{4}^{2}+l_{5}^{2}-l_{1}^{2}\right)\right]=0,
\end{aligned}
$$


and relates the squared edge lengths to the derivatives (or, more properly here, finite differences) of the scalar field.

\section{Gravitational functional measure}

In this section we will re-examine the issue of the gravitational functional measure in light of the results of the previous sections, and in particular the local gauge invariance of the lattice gravitational action. It is well known that in ordinary (lattice) gauge theories the invariance of the action selects a unique measure (the group invariant Haar measure). A natural way to construct the gravitational functional measure in the continuum is to introduce a metric over metrics (or super-metric), and then compute the resulting functional volume element. We shall see that what appears at first as a rather straightforward procedure is in fact affected by a number of rather subtle ambiguities.

\subsection{Continuum case}

Following DeWitt [47], one introduces a super-metric $G$ over metric deformations $\delta g_{\mu \nu}(x)$, which in the simplest local form leads to the following norm-squared for metric deformations:

$$
\|\delta g\|^{2} \equiv \int d^{d} x G^{\mu \nu, \alpha \beta}[g(x)] \delta g_{\mu \nu}(x) \delta g_{\alpha \beta}(x),
$$

with the inverse of the DeWitt supermetric given by

$$
G^{\mu \nu, \alpha \beta}[g(x)]=\frac{1}{2} \sqrt{g(x)}\left[g^{\mu \alpha}(x) g^{\nu \beta}(x)+g^{\mu \beta}(x) g^{\nu \alpha}(x)+\lambda g^{\mu \nu}(x) g^{\alpha \beta}(x)\right],
$$

and $\lambda \neq-2 / d$, to avoid the vanishing of the determinant of $G$. It is easy to check that the above expression for $\|\delta g\|^{2}$ is invariant under diffeomorphisms. ${ }^{6}$ The usual procedure is then to derive the functional measure in the form

$$
\int d \mu[g]=\int \prod_{x}(\operatorname{det}[G(g(x))])^{1 / 2} \prod_{\mu \geqslant \nu} d g_{\mu \nu}(x),
$$

with the determinant of the super-metric $G^{\mu \nu, \alpha \beta}(g(x))$ given by

$$
\operatorname{det} G(g(x)) \propto\left(1+\frac{1}{2} d \lambda\right)[g(x)]^{(d-4)(d+1) / 4} .
$$

Up to irrelevant constants, in four dimensions it reduces to the very simple expression

\footnotetext{
${ }^{6}$ While it should be clear that the functional norm should be invariant, it is less obvious that it should be local. Ultimately the justification for locality lies in the fact that the resulting functional measure is local in the continuum, a rather desirable feature.
} 


$$
\int d \mu[g]=\int \prod_{x}[g(x)]^{(d-4)(d+1) / 8} \prod_{\mu \geqslant \nu} d g_{\mu \nu}(x) \underset{d=4}{\rightarrow} \int \prod_{x} \prod_{\mu \geqslant \nu} d g_{\mu \nu}(x) .
$$

Unfortunately this measure is not gauge invariant, if the product over $x$ is interpreted as one over 'physical' points, and coordinate invariance is imposed at one and the same 'physical' point, as discussed in [48]. Here this is seen as a consequence of the fact that $\|\delta g\|^{2}$ has been split in two separately non-invariant parts. The measure that does satisfies the invariance property is

$$
\int d \mu[g]=\int \prod_{x}(\operatorname{det}[G(g(x)) / \sqrt{g(x)}])^{1 / 2} \prod_{\mu \geqslant \nu} d g_{\mu \nu}(x),
$$

and was originally proposed by Misner [49]. Explicitly,

$$
\int d \mu[g]=\int \prod_{x}(g(x))^{-(d+1) / 2} \prod_{\mu \geqslant \nu} d g_{\mu \nu}(x) .
$$

It has the property of being scale invariant in any dimension. Unfortunately it is also singular, and needs therefore to be regulated in some way for small $g$ (on the lattice this requires some cutoff for small local volumes).

Indeed both measures can be obtained as particular cases if one introduces a real parameter $\omega$, and writes

$$
\|\delta g\|^{2}=\int d^{d} x(g(x))^{\omega / 2} G^{\mu \nu, \alpha \beta}[g(x) ; \omega] \delta g_{\mu \nu}(x) \delta g_{\alpha \beta}(x),
$$

with

$$
\begin{aligned}
G^{\mu \nu, \alpha \beta}[g(x) ; \omega]= & \frac{1}{2}(g(x))^{(1-\omega) / 2} \\
& \times\left[g^{\mu \alpha}(x) g^{\nu \beta}(x)+g^{\mu \beta}(x) g^{\nu \alpha}(x)+\lambda g^{\mu \nu}(x) g^{\alpha \beta}(x)\right] .
\end{aligned}
$$

The metric in function space is obviously left unchanged by this rewriting, but the measure (obtained from $\operatorname{det} G$ ) depends on $\omega$, and by the usual argument one obtains the parameterized functional gravitational measure

$$
\int d \mu[g]=\int \prod_{x}[\sqrt{g(x)}]^{\sigma} \prod_{\mu \geqslant \nu} g_{\mu \nu}(x)
$$

with the measure parameter $\sigma$ related to the choice of $\omega$ via

$$
\sigma=-(d+1)+(\omega-1) \frac{d(d+1)}{4}
$$

For $\omega=0$ one obtains the DeWitt measure of Eq. (6.5), while for $\omega=1$ one has the Misner measure of Eq. (6.7). The close relationship between the DeWitt and Misner 
measures was pointed out in [50]. 7

As there is no way of deciding between these two choices, or any intermediate one for that matter, one is forced to consider $\sigma$ as an arbitrary (and hopefully ultimately irrelevant) real parameter of the theory, the only constraint being $\sigma>-(d+1)$. In general the volume factor $g^{\sigma / 2}$ in the functional measure is absent in $d$ dimensions for the special choice $\omega=1+4 / d$. It should be emphasized here that gauge invariance does not select a specific value for $\sigma$, but does otherwise completely constrain the form of the measure. The criteria of simplicity and universality would suggest the above to be the preferred choice for $\omega$. The reason for the ambiguity in the gravitational functional measure appears to be a lack of a clear definition of what is meant by $\prod_{x}$. In spite of some recurrent claims to the contrary, such an ambiguity persists in all lattice formulations. Also, the volume term in the measure is completely local since it contains no derivatives. It does not effect the propagation of gravitons, as it contributes $\delta^{d}(0)$ terms to the effective action. To some extent these can be regarded as renormalizations of the cosmological constant, since they clearly only affect the distribution of local volumes. As such they are expected to only affect the short distance behavior of the theory, leaving the more interesting universal large-distance properties unmodified (the recent work in Ref. [51] gives a more concrete realization of these ideas in the framework of continuum perturbation theory). Support to this interpretation also comes from a number of simple examples [38].

\subsection{Lattice transcription}

Let us examine the consequences of this discussion in the discrete case. As the edge lengths play the role of the metric in the continuum, one expects the discrete measure to involve an integration over edge lengths [3,5]. Indeed the induced metric at a simplex is related to the edge lengths squared within that simplex, via the expression for the invariant line element $d s^{2}=g_{\mu \nu} d x^{\mu} d x^{\nu}$. The relation between metric perturbations and squared edge length variations for a given simplex in $d$ dimensions is (see Eqs. (2.7) and (3.39), and Fig. 3)

$$
\delta g_{i j}\left(l^{2}\right)=\frac{1}{2}\left(\delta l_{0 i}^{2}+\delta l_{0 j}^{2}-\delta l_{i j}^{2}\right)
$$

Thus for one simplex the integration over the metric is equivalent to an integration over the edge lengths, and one has

\footnotetext{
${ }^{7}$ The gravitational measure has to be modified in the presence of the matter fields. For an $n$-component massless scalar field the invariant measure is$$
\int d \mu[\phi]=\int \prod_{x} g^{n / 4}(x) \prod_{a=1}^{n} d \phi^{a}(x),
$$

and therefore $\sigma=-(d+1)+(\omega-1) \frac{d(d+1)}{4}+n / 2$.
} 


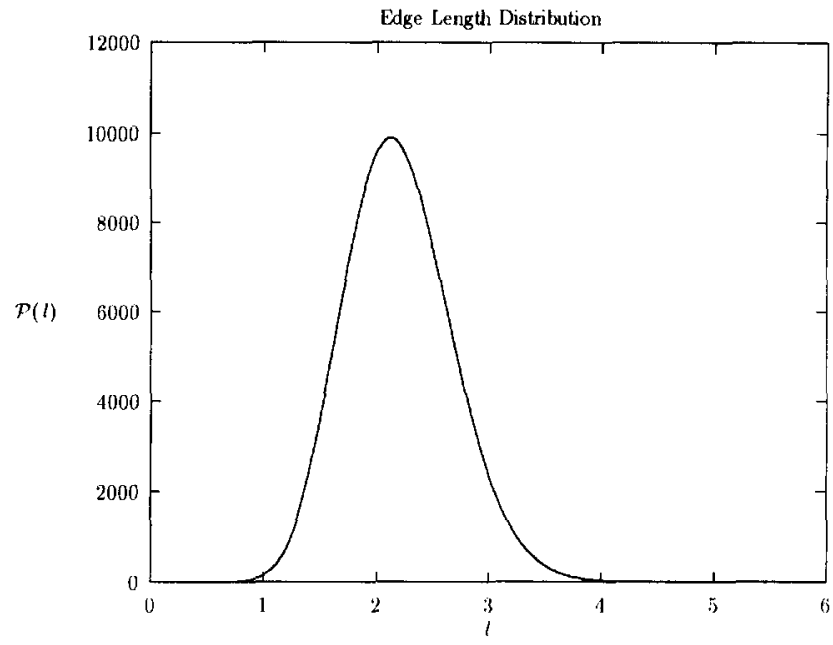

Fig. 21. Typical edge length distribution $\mathcal{P}(l)$ in four dimensions, for the lattice analog of the DeWitt measure $\sigma=0$ (see Eq. (6.15)), close to the critical point at $G_{c}$ (from Ref. [9]).

$$
\left(\frac{1}{d !} \sqrt{\operatorname{det} g_{i j}(s)}\right)^{\sigma} \prod_{i \geqslant j} d g_{i j}(s)=\left(-\frac{1}{2}\right)^{d(d-1) / 2}\left[V_{d}\left(l^{2}\right)\right]^{\sigma} \prod_{k=1}^{d(d+1) / 2} d l_{k}^{2} .
$$

There are $d(d+1) / 2$ edges for each simplex, just as there are $d(d+1) / 2$-independent components for the metric tensor in $d$ dimensions. (We are ignoring for the moment the triangle inequality constraints, which further require all sub-determinants of $g_{i j}$ to be positive as well, including the obvious restriction $l_{k}^{2}>0$.) The extension to many simplices glued together at their common faces is then immediate, and after summing over all simplices one obtains, up to an irrelevant numerical constant,

$$
\int d \mu\left[l^{2}\right]=\int_{0}^{\infty} \prod_{s}\left[V_{d}(s)\right]^{\sigma} \prod_{i j} d l_{i j}^{2}
$$

In four dimensions the lattice DeWitt measure $(\sigma=0)$ is particularly simple,

$$
\int d \mu\left[l^{2}\right]=\int_{0}^{\infty} \prod_{i j} d l_{i j}^{2} F\left[l_{i j}^{2}\right]
$$

Here $F_{\epsilon}[l]$ is a (step) function of the edge lengths, with the property that it is equal to one whenever the triangle inequalities and their higher-dimensional analogs are satisfied, and zero otherwise. ${ }^{8}$

The above lattice measure over edge lengths has recently been used extensively in numerical simulations of simplicial gravity [8-10,15,33,52,53] (Fig. 21 gives an

\footnotetext{
${ }^{8}$ The functional measure over edge lengths in Eq. (6.16) does not have compact support, and a cosmological term (with coefficient $\lambda>0$ ) is therefore essential in obtaining convergence of the functional integral for large edge lengths.
} 
example of the edge length distribution obtained in four dimensions with measure parameter $\sigma=0$ ). One would expect that physical properties of the theory should not depend on the arbitrary parameter $\sigma$, but for the moment this universality argument remains largely an unproven conjecture. The universality argument is in part tenuously supported by some systematic numerical studies in two [30] and three [6] dimensions, although there is no solid evidence for it yet in four dimensions $[9,10]$. The authors of Ref. [10] have also independently emphasized the importance of exploring the effects of different choices for the measure parameter $\sigma$. In the context of dynamical triangulations and their relationship with simplicial gravity, the irrelevance of the measure parameter has also been proposed in [55]. Finally let us mention that one can argue backwards [54] that the Regge lattice measure of Eqs. (6.14) and (6.16) provides further support for the correctness of the continuum DeWitt functional measure approach.

The above measure can also be obtained by considering a simplicial analog of the DeWitt supermetric, as was suggested in $[27,28]$. One writes for the induced metric of Eq. (2.7)

$$
\|\delta g(s)\|^{2}=\sum_{s} G^{i j k l}(g(s)) \delta g_{i j}(s) \delta g_{k l}(s),
$$

with the inverse of the lattice DeWitt supermetric given now by

$$
G^{i j k l}[g(s)]=\frac{1}{2} \sqrt{g(s)}\left[g^{i k}(s) g^{j l}(s)+g^{i l}(s) g^{j k}(s)+\lambda g^{i j}(s) g^{k l}(s)\right],
$$

and $(\lambda \neq-2 / d)$. This defines a metric on the tangent space of positive real symmetric matrices $g$. The resulting functional measure is the one of Eq. (6.15), with, by construction, $\sigma=(d-4)(d+1) / 4$, the DeWitt value. Thus

$$
\int d \mu\left[l^{2}\right]=\int \prod_{s}[\operatorname{det} G(g(s))]^{1 / 2} \prod_{i \geqslant j} d g_{i j}(s),
$$

with the determinant of the super-metric $G^{i j k l}(g(s))$ given by

$$
\operatorname{det} G(g(s)) \propto\left(1+\frac{1}{2} d \lambda\right)[g(s)]^{(d-4)(d+1) / 4},
$$

and therefore up to irrelevant constants

$$
\int d \mu\left[l^{2}\right]=\int_{0}^{\infty} \prod_{s}[V(s)]^{\sigma} \prod_{i j} d l_{i j}^{2},
$$

with $\sigma=(d-4)(d+1) / 4$. The measure factor can be exponentiated and written equivalently as an effective action contribution

$$
\int d \mu\left[l^{2}\right]=\int_{0}^{\infty} \prod_{i j} d l_{i j}^{2} \exp \left\{\sigma \sum_{s} \log V(s)\right\} .
$$

Its effect is to suppress or enhance, depending on the sign of $\sigma$, contributions from small volumes. As such, it acts much like a cosmological constant contribution 


$$
\exp \left\{-\lambda \sum_{s} V(s)\right\} \text {. }
$$

\subsection{Lund Regge approach}

The previous approach to the functional measure is based on a direct discretization of the continuum measure, and leads to a unique local measure over the squared edge lengths (modulo the volume factors), in close analogy to the continuum expression. Alternatively, one can try to find a discrete form for the supermetric, and then evaluate the resulting determinant.

In a paper Lund and Regge offered a slightly different approach to the measure problem [45], in connection with the $3+1$ formulation of simplicial gravity. The idea, recently re-analyzed by the authors of Ref. [46], was to obtain a lattice analog of the DeWitt supermetric, by considering the quantity

$$
\left\|\delta l^{2}\right\|^{2}=\sum_{i j} G_{i j}\left(l^{2}\right) \delta l_{i}^{2} \delta l_{j}^{2},
$$

where $G_{i j}\left(l^{2}\right)$ plays a role analogous to the DeWitt supermetric, but now on the space of squared edge lengths. One way of constructing the explicit form for $G_{i j}\left(l^{2}\right)$ is to write the squared volume of a given simplex in terms of the induced metric within the same simplex,

$$
V^{2}(s)=\left(\frac{1}{d !}\right)^{2} \operatorname{det}\left\{g_{i j}\left(l^{2}(s)\right)\right\} .
$$

Then compare the expansion of the determinant of the metric in the continuum,

$$
\begin{aligned}
\operatorname{det}\left(g_{i j}+\delta g_{i j}\right) & =\exp \operatorname{Tr} \log \left(g_{i j}+\delta g_{i j}\right) \\
& =\operatorname{det}\left(g_{i j}\right)\left[1+g^{i j} \delta g_{i j}+\frac{1}{2} g^{i j} g^{k l} \delta g_{i j} \delta g_{k l}-\frac{1}{2} g^{i j} g^{k l} \delta g_{j k} \delta g_{l i}+\ldots\right],
\end{aligned}
$$

to the analogous expansion for the square of the volume of a simplex

$$
V^{2}\left(l^{2}+\delta l^{2}\right)=V^{2}\left(l^{2}\right)+\sum_{i} \frac{\partial V^{2}\left(l^{2}\right)}{\partial l_{i}^{2}} \delta l_{i}^{2}+\frac{1}{2} \sum_{i j} \frac{\partial^{2} V^{2}\left(l^{2}\right)}{\partial l_{i}^{2} \partial l_{j}^{2}} \delta l_{i}^{2} \delta l_{j}^{2}+\ldots
$$

Identifying terms of order $\left(\delta l_{i}^{2}\right)^{n}$ in Eq. (6.27) with terms of order $\left(\delta g_{i j}\right)^{n}$ in Eq. $(6.26)$ one obtains to linear order

$$
\frac{1}{V\left(l^{2}\right)} \frac{\partial V^{2}\left(l^{2}\right)}{\partial l_{i}^{2}} \delta l_{i}^{2}=\frac{1}{d !} \sqrt{\operatorname{det}\left(g_{i j}\right)} g^{i j} \delta g_{i j},
$$

and to quadratic order

$$
\frac{1}{V\left(l^{2}\right)} \sum_{i j} \frac{\partial^{2} V^{2}\left(l^{2}\right)}{\partial l_{i}^{2} \partial l_{j}^{2}} \delta l_{i}^{2} \delta l_{j}^{2}=\frac{1}{d !} \sqrt{\operatorname{det}\left(g_{i j}\right)}\left[g^{i j} g^{k l} \delta g_{i j} \delta g_{k l}-g^{i j} g^{k l} \delta g_{j k} \delta g_{l i}\right] \text {. }
$$


Remarkably, the right-hand side of this equation contains precisely the expression appearing in the continuum supermetric of Eq. (6.2), for the specific choice $\lambda=-2$. After summing over simplices one obtains

$$
\begin{aligned}
& \frac{1}{2} \sum_{s} \sqrt{\operatorname{det}\left(g_{i j}(s)\right)}\left[g^{i k}(s) g^{j l}(s)+g^{i l}(s) g^{j k}(s)-2 g^{i j}(s) g^{k l}(s)\right] \delta g_{i j}(s) \delta g_{k l}(s) \\
& \quad=\sum_{i j} G_{i j}\left(l^{2}\right) \delta l_{i}^{2} \delta l_{j}^{2}
\end{aligned}
$$

with

$$
G_{i j}\left(l^{2}\right)=-d ! \sum_{s} \frac{1}{V(s)} \frac{\partial^{2} V^{2}(s)}{\partial l_{i}^{2} \partial l_{j}^{2}},
$$

which now determines the matrix $G_{i j}\left(l^{2}\right)$ appearing in the Lund-Regge metric for deformations in the space of squared edge lengths, Eq. (6.24). The analogy with the continuum expression is brought out more clearly when one factors out the volume element, and writes

$$
\left\|\delta l^{2}\right\|^{2}=\sum_{s} V(s)\left\{-\frac{d !}{V^{2}(s)} \sum_{i j} \frac{\partial^{2} V^{2}(s)}{\partial l_{i}^{2} \partial l_{j}^{2}} \delta l_{i}^{2} \delta l_{j}^{2}\right\} .
$$

The volume factor ambiguity present in the continuum measure is not removed though. As in the continuum, different measures on the edge lengths are obtained, depending on whether the local volume factor $V(s)$ is included in the supermetric or not. In parallel with $\mathrm{Eq}$. (6.9) one writes therefore

$$
\left\|\delta l^{2}\right\|^{2}=\sum_{s}[V(s)]^{\omega^{\prime}}\left\{-\frac{d !}{[V(s)]^{1+\omega^{\prime}}} \sum_{i j} \frac{\partial^{2} V^{2}(s)}{\partial l_{i}^{2} \partial l_{j}^{2}} \delta l_{i}^{2} \delta l_{j}^{2}\right\}
$$

The metric in edge length space is again left unchanged by this rewriting, but the measure obtained from det $G$ depends on a parameter $\omega^{\prime}$, and by the usual arguments one obtains a parameterized functional gravitational measure

$$
\int d \mu\left[l^{2}\right]=\int \prod_{i} \sqrt{\operatorname{det} G_{i j}^{\left(\omega^{\prime}\right)}\left(l^{2}\right)} d l_{i}^{2}
$$

with

$$
G_{i j}^{\left(\omega^{\prime}\right)}\left(l^{2}\right)=-d ! \sum_{s} \frac{1}{[V(s)]^{1+\omega^{\prime}}} \frac{\partial^{2} V^{2}(s)}{\partial l_{i}^{2} \partial l_{j}^{2}} .
$$

Thus the matrix $G_{i j}^{\left(\omega^{\prime}\right)}$ should be thought of as defining a one-parameter family of measures.

A somewhat disturbing feature of the Lund-Regge lattice measure of Eq. (6.34) is that it does not give the correct result already in one dimension [38]. The onedimensional action for pure gravity of Eq. (3.46), proportional to the length of the curve, is invariant under the local gauge transformations of Eq. (3.47), 


$$
\delta l_{n}=\chi_{n+1}-\chi_{n},
$$

where the $\chi_{n}$ 's represent continuous parameters defined on the lattice vertices. Any local variations of the edges which have the above form (and, we should add, also do not violate the constraint $l_{n}>0$ ) obviously leave the physical length of the curve unchanged. The invariant measure in one dimension is therefore

$$
\int d \mu\left[l^{2}\right]=\int_{0}^{\infty} \prod_{i=0}^{N} d l_{i},
$$

which falls precisely in the class of measures encompassed by Eq. (6.15), with $\sigma=-1$. On the other hand, the arguments leading to the measure of Eq. (6.34) give $l^{2}=g$ to zeroth order, $\delta l^{2} / l^{2}=\delta g / g$ to first order, and $0=0$ to second order, and therefore, since $G_{n m}=0,\left\|\delta l^{2}\right\|^{2}=0$ ! Incidentally, in one dimension a physically motivated invariant distance between manifolds is $d^{2}\left(l, l^{\prime}\right) \equiv\left[L(l)-L^{\prime}\left(l^{\prime}\right)\right]^{2}$, which is non-local.

Another somewhat undesirable feature of the Lund-Regge metric is that in general it is non-local, in spite of the fact that the original continuum measure of Eq. (6.10) is completely local. ${ }^{9}$ After all, metric perturbations and squared edge lengths are linearly related to each other, and locality for one measure should translate into locality for the other measure. Non-local contribution to the original (non-gauge-fixed) measure seem as unattractive as non-local action contributions. A non-local measure makes it virtually impossible to study the theory non-perturbatively. On the other hand it is clear that, for some special choices of $\omega^{\prime}$ and $d$, one does recover a local measure. Thus in two dimensions for $\omega^{\prime}=-1$ one obtains again the simple result

$$
\int d \mu\left[l^{2}\right]=\int_{0}^{\infty} \prod_{i} d l_{i}^{2}
$$

(which incidentally is non-singular at small edge lengths and represents therefore in this respect an acceptable measure). This measure appears therefore, on the basis of purely theoretical arguments, to be as good as any other measure with a different $\omega^{\prime}$. Given the possibility of a choice for $\omega^{\prime}$, it would seem natural that one should chose its value in such a way that the measure has the simplest form, here the local form without any volume factors: after all the physical theory should not depend on the bare parameter $\omega^{\prime}$.

We should also remark that the appearance of non-local measure contributions (and in particular for some values of the parameter $\omega^{\prime}$, but not for others) makes one question the initial justification for starting in the first place with an expression, such as the one in

\footnotetext{
${ }^{9}$ After imposing a gauge condition, such as the conformal gauge, the measure can become non-local as in ordinary gauge theories. But such a gauge-fixing term is only necessary in perturbation theory to remove the zero-modes of the action, discussed in Sections 3 and 4 . Non-perturbatively one would expect that no gauge fixing is necessary, as the effects of the gauge zero-modes are expected to cancel out in averages of physical quantities, as in ordinary lattice gauge theories [5].
} 
Eq. (6.18), which is local. In conclusion it appears that the local measure of Eq. (6.16) provides the simplest theoretically justifiable starting point, if not the only possible one.

\section{Gauge fixing and lattice conformal gauge}

Regge's simplicial quantum gravity does not require gauge fixing $[3,5]$, unless one intends to perform a diagrammatic perturbative expansion on the lattice [18]. In this respect, the situation is completely analogous to ordinary gauge theories, and one expects the volume of the gauge group, the diffeomorphism group in the case of gravity, eventually to cancel out in the expression for physical averages,

$$
\langle O\rangle=\frac{\int d \mu\left[l^{2}\right] O\left(l^{2}\right) \exp \left\{-I\left[l^{2}\right]\right\}}{\int d \mu\left[l^{2}\right] \exp \left\{-I\left[l^{2}\right]\right\}} .
$$

The lattice diffeomorphism zero-modes discussed here (see Eq. (3.21) and subsequent expressions) do not therefore in principle pose a problem in non-perturbative studies of quantized gravity, such as the ones presented in $[9,15]$. Zero-modes are automatically taken into account as the measure explores gauge-equivalent choices of metrics. One important distinction with the formal continuum theory is the presence of a cutoff in orbit space, due to the enforcement of the triangle inequalities. As a result, the gravitational functional measure is highly non-trivial. Such a constraint is not seen to any order in the perturbative weak-field expansion, it is a genuinely non-perturbative constraint.

On the other hand, in two dimensions the continuum theory can be studied by perturbative methods, which are most suitably applied in the conformal gauge [36]. It is the purpose of this section to elaborate on the connection between the continuum and the lattice theory, both being formulated here in a particular gauge. Let us first summarize the results in the continuum. The weak-field expansion is used, and one sets as usual

$$
g_{\mu \nu}(x)=\delta_{\mu \nu}+\kappa h_{\mu \nu}(x) .
$$

As there is no small parameter in two dimensions to play with, one assumes $\kappa \ll 1$, expands, and then sets $\kappa=1$ at the end. The easiest quantity to compute is the "graviton" vacuum polarization due to one massless scalar particle, a one-loop diagram here. It is given by

$$
\begin{aligned}
\Pi_{\mu \nu, \alpha \beta}(q) & =\frac{1}{2} \int \frac{d^{2} p}{(2 \pi)^{2}} \frac{t_{\mu \nu}(p, q) t_{\alpha \beta}(p, q)}{p^{2}(p+q)^{2}}, \\
t_{\mu \nu}(p, q) & =\frac{1}{2}\left[\delta_{\mu \nu} p \cdot(p+q)-p_{\mu}\left(p_{\nu}+q_{\nu}\right)-p_{\nu}\left(p_{\mu}+q_{\mu}\right)\right] .
\end{aligned}
$$

The calculation of the integral is easily done using dimensional regularization [18], or by the methods of [37]. In either case one obtains

$$
\Pi_{\mu \nu, \alpha \beta}(q)=\frac{1}{48 \pi}\left(q^{2} \delta_{\mu \nu}-q_{\mu} q_{\nu}\right) \frac{1}{q^{2}}\left(q^{2} \delta_{\alpha \beta}-q_{\alpha} q_{\beta}\right) .
$$


For a $D$-component scalar field, the above result is simply multiplied by a factor of $D$, and the effective action, to lowest order in the weak-field expansion, is then

$$
I_{\mathrm{eff}}=-\frac{1}{2} \int \frac{d^{2} q}{(2 \pi)^{2}} h_{\mu \nu}(q) \Pi_{\mu \nu \rho \sigma}(q) h_{\rho \sigma}(-q)
$$

In the conformal gauge, coordinates are chosen which are locally orthogonal, so as to bring the metric into the form

$$
g_{\mu \nu}(x)=\delta_{\mu \nu} e^{\varphi(x)} .
$$

Then one has for the scalar curvature

$$
R(q)=\left(q_{\mu} q_{\nu}-\delta_{\mu \nu} q^{2}\right) h_{\mu \nu}(q)=q^{2} \varphi(q),
$$

and one can therefore rewrite the effective action in the form

$$
\begin{aligned}
I_{\mathrm{eff}}(\varphi) & =-\frac{D}{96 \pi} \int \frac{d^{2} q}{(2 \pi)^{2}} \varphi(q) q^{2} \varphi(-q) \\
& =-\frac{D}{96 \pi} \int d^{2} x\left[\left(\partial_{\mu} \varphi\right)^{2}+\left(\lambda-\lambda_{c}\right) e^{\varphi}\right] .
\end{aligned}
$$

On the lattice one can perform a similar computation, using again perturbation theory [18]. The lattice Feynman rules are written down, the integration over the scalar is performed, and an effective action results, which can be expanded out in the weak-field limit. As the scalar couples invariantly to the gravitational degrees of freedom, one would expect that the result should be eventually expressible in terms of invariants. Indeed in the continuum one can rewrite the effective action of Eq. (7.8) in an invariant form,

$$
\frac{1}{2} \int d^{2} x d^{2} y R \sqrt{g}(x)\left\langle x\left|\frac{1}{-\partial^{2}}\right| y\right\rangle R \sqrt{g}(y),
$$

where $\partial^{2}$ is the continuum covariant Laplacian, $\partial^{2} \equiv \partial_{\mu} \sqrt{g} g^{\mu \nu} \partial_{\nu}$. On the lattice this expression has an obvious invariant counterpart [11],

$$
\frac{1}{2} \sum_{\text {hinges } h, h^{\prime}} \delta_{h}\left[\frac{1}{-\Delta}\right]_{h, h^{\prime}} \delta_{h^{\prime}},
$$

which is obtained from the correspondence between lattice and continuum curvatures derived in [7]. $\Delta$ here is the nearest-neighbor covariant lattice Laplacian, as obtained from the discrete scalar action (see Eqs. (5.9) and (5.48)); for a recent discussion of the discretization of this term see also Ref. [56]. It introduces an effective long-range interaction between deficit angles. For this reason it is actually preferable to study nonperturbative aspects of the model leaving the scalar fields un-integrated, which keeps the action local [30]. ${ }^{10}$

\footnotetext{
${ }^{10}$ It is encouraging that the conformal mode stays massless in the full non-perturbative treatment of the two-dimensional simplicial lattice theory, without the necessity of any sort of fine-tuning of bare parameters $[30,31]$.
} 
The previous discussion provides a background for motivating the introduction of the conformal gauge on the lattice. It is legitimate to ask therefore what is the simplicial lattice analog of the gauge condition of Eq. (7.6). The conformal gauge implies a local choice of orthogonal coordinates. It is clear from the discussion in Section 3.1 that there is a corresponding choice on the lattice. Indeed in the development of the weakfield expansion a uniform orthogonal set of coordinates was chosen, with a diagonal background metric (with edge length assignments $l_{i}^{0}=1$ for the body principals ( $i=$ 1,2 ) and $l_{i}^{0}=\sqrt{2}$ for the diagonal $(i=3)$ ). For these coordinates (see Eq. (3.16)) one has for the background metric

$$
g_{i j}^{(0)}=\delta_{i j}
$$

The lattice conformal gauge choice corresponds to an assignment of edge lengths such that locally

$$
\begin{aligned}
g_{i j}(n) & =\left(\begin{array}{cc}
l_{1}^{2}(n) & \frac{1}{2}\left(l_{3}^{2}(n)-l_{1}^{2}(n)-l_{2}^{2}(n)\right) \\
\frac{1}{2}\left(l_{3}^{2}(n)-l_{1}^{2}(n)-l_{2}^{2}(n)\right) & l_{2}^{2}(n)
\end{array}\right) \\
& \approx \delta_{i j} e^{\varphi(n)} .
\end{aligned}
$$

Here the lattice fields $\varphi(n)$ have to be defined on the lattice vertices, and so are the gauge degrees of freedom $\chi_{\mu}(n)$, as can be inferred from Eq. (3.23). It is clear therefore that a choice of lattice conformal gauge corresponds to a re-assignment of edge lengths about each vertex, in such a way that the local curvature is left unchanged, but at the same time the induced metric is brought into diagonal form; it corresponds to a choice of approximately right-angle triangles at each lattice vertex.

This result is further illustrated in Figs. 22-24. The surface shown in Fig. 22 has been brought into the lattice analog of the conformal gauge, by re-assigning edge lengths in such a way that individual triangles look as close as possible to right-angle triangles. In going from Fig. 24 to Fig. 23, repeated gauge transformations must be performed on the vertices, by reassigning edge lengths in such a way that local areas and volumes are kept unchanged. It is easy to see that such a construction can always be done, except in some rather pathological cases.

The gravitational contribution to the effective action in the lattice conformal gauge can, at least in principle, be computed in a similar way. Let us sketch here how the analogous lattice calculation would proceed; a more detailed discussion will be presented elsewhere. In the continuum the metric perturbations are naturally decomposed into orthogonal conformal and diffeomorphism parts,

$$
\delta g_{\mu \nu}(x)=g_{\mu \nu}(x) \delta \varphi(x)+\nabla_{\mu \chi \nu}(x)+\nabla_{\nu} \chi_{\mu}(x),
$$

where $\nabla_{\nu}$ denotes the covariant derivative. It should be clear from the discussion in Section 3 that a rather similar decomposition can be done for the lattice degrees of freedom, by separating out the lattice gauge transformations (which act on the vertices and change the edge lengths without changing the local volumes and curvatures) from 


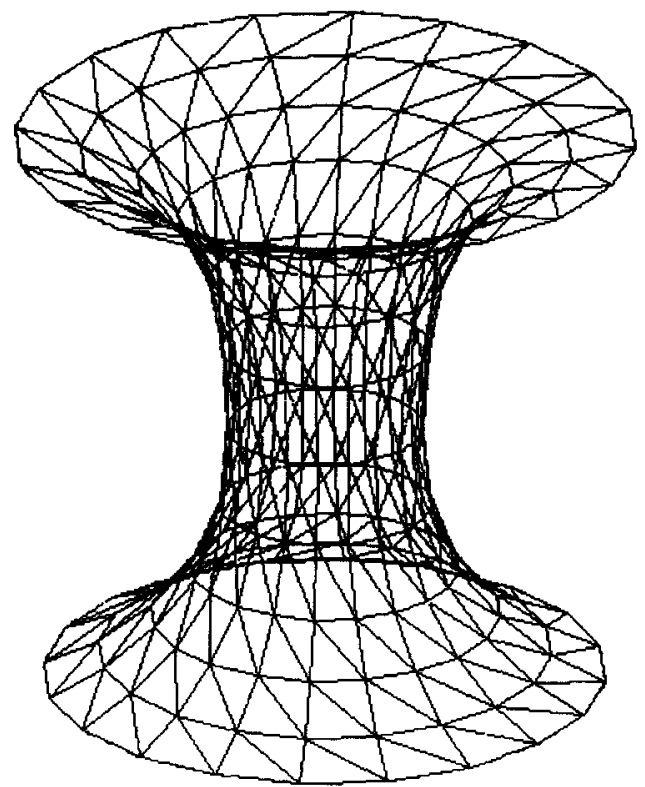

Fig. 22. Description of a smooth surface in the lattice analog of the conformal gauge.

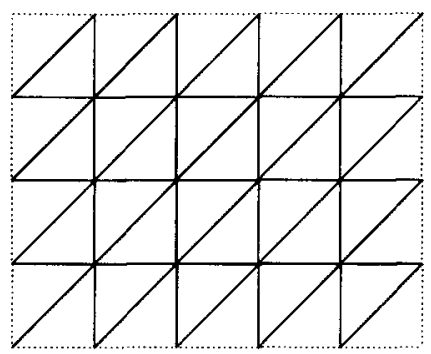

Fig. 23. Enlarged view of a small region on the surface in Fig. 22.

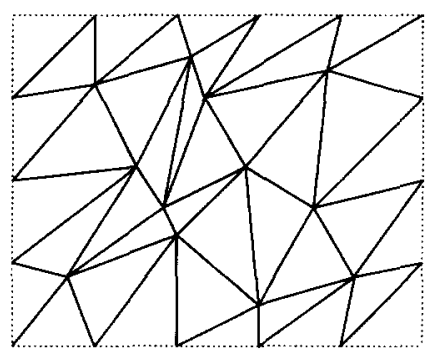

Fig. 24. Gauge equivalent description of the enlarged view, of a small region of the original surface, represented in Fig. 22.

the conformal transformations (which do change them) in Eq. (3.39). The explicit form for the lattice diffeomorphisms, to lowest order in the lattice weak-field expansion, is given in Eq. (3.21), while the explicit form for the lattice conformal transformations in given in Eq. (3.27), which makes it obvious that such a decomposition can indeed be 
performed on the lattice. In the continuum, after rewriting the gravitational functional measure in terms of conformal and diffeomorphism degrees of freedom,

$$
\int d \mu[g]=\int d \mu[\varphi] d \mu[\chi]\left[\operatorname{det}\left(L^{+} L\right)\right]^{1 / 2},
$$

one has to compute the Jacobian of the operator $L$. It is determined, in the continuum, from

$$
\left(L^{+} L \chi\right)_{\mu}=\nabla^{\nu}\left(\nabla_{\mu} \chi_{\nu}+\nabla_{\nu} \chi_{\mu}-g_{\mu \nu} \nabla^{\rho} \chi_{\rho}\right)
$$

One then obtains for the effective action contribution in the conformal gauge,

$$
\left[\operatorname{det}\left(L^{+} L\right)\right]^{-1 / 2} \sim \exp \left\{-I_{\mathrm{eff}}(\varphi)\right\}
$$

with $I_{\text {eff }}$ of the form in Eq. (7.5) to lowest order in the weak-field expansion. A diagrammatic calculation, similar to the one for the scalar field contribution, gives in the continuum the celebrated result $[36,37]$

$$
\Pi_{\mu \nu \rho \sigma}(q)=\frac{13}{48 \pi}\left(q_{\mu} q_{\nu}-\delta_{\mu \nu} q^{2}\right) \frac{1}{q^{2}}\left(q_{\rho} q_{\sigma}-\delta_{\rho \sigma} q^{2}\right) .
$$

On the lattice the functional integration is performed over the squared edge lengths, as in Eq. (6.15). But it seems a technically challenging task to compute the Jacobian that maps the edge length variables (which define the lattice metric) to the orthogonal lattice diffeomorphism variables of Eq. (3.23) and the lattice conformal fields of Eq. (3.27), with the appropriate Jacobian included. It is also quite possible that one might have to go beyond the lowest order in the lattice perturbative expansion.

As a consequence the total Liouville action for the Liouville field $\varphi=\frac{1}{\lambda^{2}} R$ becomes

$$
I_{\mathrm{eff}}(\varphi)=\frac{26-D}{96 \pi} \int d^{2} x\left[\left(\partial_{\mu} \varphi\right)^{2}+\left(\lambda-\lambda_{c}\right) e^{\varphi}\right] .
$$

To lowest order in the weak-field expansion the critical value of $D$ for which the action vanishes is $D_{c}=26$, but this number is modified by higher order quantum corrections. In any case, for sufficiently large $D$ one expects an instability to develop. Numerical non-perturbative studies of two-dimensional gravity suggest that in the lattice theory the correction is large, and one finds that the threshold of instability moves to values as low as $D_{c} \approx 13$ [31]. It is unclear whether this critical value can be regarded as truly universal, and independent for example on the detailed choice of gravitational measure (we are referring here for example to the choice of parameter $\sigma$ ).

\section{Conclusions}

We have shown in this paper that Regge's formulation of simplicial gravity is endowed with a remnant of the continuous local gauge invariance of the original continuum theory. The appearance of zero-modes corresponding to the diffeomorphisms in the continuum is 
particularly transparent in the weak-field expansion. Nevertheless, the presence of a local invariance in the discrete action can be exhibited, via the detailed explicit calculations presented in this paper, for almost any conceivable choice of background lattice. It was shown in particular that the structure of the zero-modes corresponds precisely to the discretized form of the diffeomorphism transformation law in the continuum. We have underscored the fact that the squared edge lengths correspond to the metric components in the continuum, and that such a result is therefore hardly surprising (indeed it has been known for some time in some special cases). Explicit calculations also show that the gauge and conformal modes are consistently defined as acting on the vertices of the lattice. Although our derivations have mainly been restricted to the two-dimensional case, where they are more transparent and one does not run the risk of drowning in a sea of indices, we have argued that they have general applicability, and in a number of cases we have indicated the structure of the general result.

Our results have a bearing on the issue of the gravitational measure in simplicial gravity. As the metric degrees of freedom in the continuum correspond to the squared edge lengths in the lattice theory, it is clear that functional integration in the latter should be performed again over the edge lengths squared. We have provided a number of arguments in support of this statement, based on DeWitt's approach to the functional measure in the continuum. We have argued that the lattice measure is essentially no less unique than the original continuum (DeWitt) measure, with ambiguities restricted to local volume factors, and which most likely are not relevant in four dimensions. It is unlikely that further insight into this issue can come from analytical work, and it is hoped that future numerical simulations will support this conclusion, for which there is already some partial and incomplete evidence. At the end of the paper we have considered the introduction of gauge-fixing terms in the lattice action, which are needed in order to remove the gauge zero-modes of the gravitational action in perturbative calculations. Again the situation is similar to what happens in the continuum when one performs perturbation theory, where one first separates out the infinite gauge volume contribution. As a specific example, we have discussed how one goes about constructing the lattice conformal gauge.

A more practical motivation for our work has been to try to understand the recently discovered discrepancy between the critical exponents for matter coupled to gravity in two dimensions as computed in the lattice regularized model for gravity [30,33], and the corresponding conformal field theory predictions for central charge $c=\frac{1}{2}$ [58,57]. Particularly significant in this respect appears to be the recent realization that the conformal field theory exponents describe two-dimensional random systems in flat space, and do not correspond to "gravitational" dressing of correlators [60] (see Table 1). Recent independent calculations have to some extent confirmed this result [61].

Previously the authors of Ref. [62] had considered the Dirac equation on a twodimensional lattice where sites have been removed randomly - a doped lattice. They argued that in this case the fermions acquire a quartic interaction and become Thirring fermions, thus changing the critical exponents and the universality class. In our opinion, 
Table 1

Critical exponents of random and non-random Ising models

\begin{tabular}{llllll}
\hline & $\gamma / \nu$ & $\beta / \nu$ & $\alpha / \nu$ & $\alpha$ & \multicolumn{1}{c}{$\nu$} \\
\hline Onsager solution on regular flat lattice & 1.75 & 0.125 & 0 & 0 & 1 \\
Ising spins coupled to gravity [30,33] & $1.73(2)$ & $0.124(3)$ & $-0.06(11)$ & - & $0.98(1)$ \\
Matrix model and CFT [57,58] & $1.333 \ldots$ & $0.333 \ldots$ & $-0.666 \ldots$ & -1.0 & 1.5 \\
Random Ising spins in flat space [60] & $1.32(3)$ & $0.31(4)$ & $-0.65(4)$ & $-0.98(4)$ & $1.46(8)$ \\
\hline
\end{tabular}

the results for simplicial gravity found in [30], and the conformal field theory exponents of [58], can simply be made consistent with each other, if an additive gravitational dressing of critical exponents is taken to be zero in both cases, for both non-random and random matter (which are known to have different critical exponents in flat space to begin with). ${ }^{11}$

\section{Acknowledgements}

The authors thank Gabriele Veneziano and the Theory Division at CERN for hospitality during the completion of this paper. The authors also acknowledge discussions with James Hartle, and thank him for presenting his ideas on the supermetric. The work of H.W.H. and R.M.W. was supported in part by the UK Science and Engineering Research Counsel under grant GR/J64788.

\section{References}

[1] S.W. Hawking, in General Relativity - An Einstein Centenary Survey, ed. S.W. Hawking and W. Israel (Cambridge Univ. Press, Cambridge, 1979).

[2] T. Regge, Nuovo Cimento 19 (1961) 558.

[3] H.W. Hamber, Simplicial Quantum Gravity, in 1984 Les Houches Summer School, Session XLIII (North-Holland, Amsterdam, 1986).

[4] M. Roðek and R.M. Williams, Phys. Lett. B 104 (1981) 31; Z. Phys. C 21 (1984) 371.

[5| J.B. Hartle, J. Math. Phys. 26 ( 1985) 804; 27 (1985) 287; 30 (1989) 452.

[6] H.W. Hamber and R.M. Williams, Phys. Rev. D 47 (1993) 510.

[7] H.W. Hamber and R.M. Williams, Nucl. Phys. B 248 (1984) 392; B 260 (1985) 747; Phys. Lett. 157B (1985) 368; Nucl. Phys. B 269 (1986) 712.

[8] B. Berg, Phys. Rev. Lett. 55 (1985) 904; Phys. Lett. B 176 (1986) 39.

[9] H.W. Hamber, UCI-TH-91-09 (Jan 1991), Nucl. Phys. B (Proc. Supp.) 20 (1991) 728; Phys. Rev. D 45 ( 1992) 507; Nucl. Phys. B 400 (1993) 347.

\footnotetext{
11 A difficult issue seems to be the determination of the string susceptibility exponent in numerical simulations. Corner points in non-homogeneous lattices give rise to spurious, non-universal $1 /$ volume contributions to the average action, and require a subtraction before a universal correction can be obtained. This apparently elementary consideration was overlooked in Ref. [63], and leads if untreated to uncontrolled systematic errors. Indeed, for $n_{a}$ interior points with coordination number $q_{a}$ and $n_{b}$ corner points with coordination number $q_{b}$ $\left(n_{a}+n_{b}=N_{0}\right)$, the average action per unit area can be written as $\langle I\rangle / A \approx\left(n_{a} I_{a}+n_{b} I_{b}\right) /\left(n_{a} A_{a}+n_{b} A_{b}\right)$. For large $N_{0},\langle I\rangle / A=I_{a} / A_{a}+c / N_{0}+O\left(1 / N_{0}^{2}\right)$ with a non-universal constant $c=\left(n_{b} / A_{a}^{2}\right)\left(I_{b} A_{a}-I_{a} A_{b}\right)$. For homogeneous lattices, such as the torus used in Ref. [30], the spurious non-universal correction vanishes, since $I_{a}=I_{b}$ and $A_{a}=A_{b}$.
} 
[10] W. Beirl, E. Gerstenmayer, H. Markum and J. Riedler, Phys. Rev. D 49 (1994) 5231;

W. Beirl, H. Markum and J. Riedler, Phys. Lett. B 341 (1994) 12.

[11] H.W. Hamber and R.M. Williams, Nucl. Phys. B 267 (1986) 482.

[12] A.M. Polyakov, PUPT-1394 (April 1993), in 1992 Les Houches Summer School, Session 57 (NorthHolland, Amsterdam, 1995).

[13| V. De Alfaro, S. Fubini and G. Furlan, Nuovo Cim. 57B (1980) 227; Phys. Lett. 97B (1980) 67; in Erice 1981 (Plenum, New York, 1983).

[14] G. Modanese, Phys. Lett. 325B (1994), 354; Nucl. Phys. B 434 (1995) 697.

[15] H.W. Hamber and R.M. Williams, Nucl. Phys. B 435 (1995) 361.

[16] R.M. Williams and P.A. Tuckey, Class. Quant. Grav. 9 (1992) 1409;

R.M. Williams, Int. J. Mod. Phys. B 6 (1992) 2097.

[17] B.V. de Bakker and J. Smit, Amsterdam Preprint AIAP-1996-043 (Apr 1996), and references therein; P. Bialas, Z. Burda, A. Krzywicki and B. Petersson, Preprint LPTHE-ORSAY-96-08 (Jan 1996);

B.V. de Bakker, Amsterdam Preprint (March 1996);

For earlier work, see M.E. Agishtein and A.A. Migdal, PUPT-1287 (Oct 1991), Mod. Phys. Lett. A 7 (1992) 1039; PUPT-1311 (Mar 1992), Nucl. Phys. B 385 (1992) 395;

J. Ambjorn and J. Jurkiewicz, NBI-HE-91-60 (Dec 1991), Phys. Lett. B 278 (1992) 42;

J. Ambjorn, J. Jurkiewicz, C.F. Kristjansen, NBI-HE-92-53 (Jul 1992) Nucl. Phys. B 393 (1993) 601;

S. Varsted, UCSD-PTH-92-03 (Jan 1992), Nucl. Phys. B 412 (1994) 406.

[18] H.W. Hamber and S. Liu, Nucl. Phys. B 472 (1996) 447.

[19] C. Itzykson, in Progress in Gauge Field Theory (Cargèse Lecture Notes 1983), published in the proceedings (Plenum, New York, 1985).

[20] H. Koibuchi and M. Jamada, Mod. Phys. Lett. A 4 (1989) 475, 1249, 2417.

[21] T.D. Lee, in 1983 Erice International School of Subnuclear Physics (Plenum, New York, 1985).

[22] N. Christ, R. Friedberg and T.D. Lee, Nucl. Phys. B 202 (1982) 89; Nucl. Phys. B 210 [FS6] (1982) $310,337$.

[23] J.M. Drouffe and C. Itzykson, Nucl. Phys. B 235 [FS11] (1984) 45.

[24] E.J. Gardner, C. Itzykson and B. Derrida, J. Phys. A 17 (1984) 1093.

[25] D. Espriu, M. Gross, P. Rakow and J.F. Wheater, Nucl. Phys. B 265 [FS15] (1986) 92;

W. Janke, M. Katoot and R. Villanova, Phys. Lett. B 315 (1993) 412.

[26] F. David, in 1993 Les Houches Summer School, Session XLIII (North-Holland, Amsterdam, 1994) pp. $375-439$, and references therein.

[27] J. Cheeger, W. Müller and R. Schrader, Comm. Math. Phys. 92 (1984) 405.

[28| J. Cheeger, W. Müller and R. Schrader, in the Heisenberg Symposium (Springer, New York, 1982).

[29] R. Friedberg and T.D. Lee, Nucl. Phys. B 242 (1984) 145;

G. Feinberg, R. Friedberg, T.D. Lee and H.C. Ren, Nucl. Phys. B 245 (1984) 343.

[30] M. Gross and H.W. Hamber, Nucl. Phys. B 364 (1991) 703.

[31] H.W. Hamber, Nucl. Phys. B (Proc. Suppl.) 25A (1992) 150-175; and in Monte Carlo Methods in Theoretical Physics (ETS Editrice, Pisa, 1992) pp. 119-144.

[32| Z. Tabor, Preprint TPJU-10-95 (April 1995), 12pp.

[33 | C. Holm and W. Janke, Phys. Lett. B 335 (1994) 143; Preprint FUB-HEP-17-95 (Nov. 1995).

134] M. Veltman, in Methods in Field Theory, Les Houches Lecture notes, Session XXVIII (North-Holland, Amsterdam, 1975).

[35] G. Bhanot and C. Rebbi, Phys. Rev. D 24 (1981) 3319.

[36] A.M. Polyakov, Phys. Lett. B 103 (1981) 207.

[37| A.M. Polyakov, Gauge Fields and Strings, ch. 9 (Oxford Univ. Press, Oxford, 1989).

[38] H.W. Hamber and R.M. Williams, Nucl. Phys. B 451 (1995) 305; see also J. Nishimura, KEK-TH-437 (Dec. 1995); T. Nakajima, UT-705 (Jan. 1996).

[39| A. Jevicki and M. Ninomiya, Phys. Rev. D 33 (1986) 1634.

[40] H.W. Hamber, in Probabilistic Methods in Quantum Field Theory and Quantum Gravity (Cargèse NATO Workshop, 1989) (Plenum, New York 1990) pp. 243-257.

[41] M. Bander and C. Itzykson, in Proc. of the Meudon Seminar 1983-4:257 (Springer, Berlin 1985); Nucl. Phys. B 257 [FS14] (1985) 531.

[42| A. Jevicki and M. Ninomiya, Phys. Lett. 150B (1985) 115.

|43| H.C. Ren, Nucl. Phys. B 301 (1988) 661. 
[44] See for example H. Kawai, R. Nakayama and K. Seo, Nucl. Phys. B 189 (1981) 40, and references therein.

[45] F. Lund and T. Regge, Princeton Preprint 1974 (unpublished).

[46] J.B. Hartle, W.A. Miller and R.M. Williams, Preprint to appear.

[47] B. DeWitt, in Dynamical Theory of Groups and Fields (Gordon and Breach, New York, 1965);

B. DeWitt, Phys. Rev. 160 (1967) 1113;

B. DeWitt, in General Relativity - An Einstein Centenary Survey, ed. S.W. Hawking and W. Israel (Cambridge Univ. Press, Cambridge, 1979);

K. Fujikawa, Nucl. Phys. B 226 (1983) 437;

K. Fujikawa and O. Yasuda, Nucl. Phys. B 245 (1984) 446;

D. Anselmi, Phys. Rev. D 45 (1992) 4473.

[48] L. Fadeev and V. Popov, Sov. Phys. Usp. 16 (1974) 777; Usp. Fiz. Nauk. 109 (1974) 427;

N.P. Konopleva and V.N. Popov, in Gauge Fields (Harwood, New York, 1979).

[49] C.W. Misner, Rev. Mod. Phys. 29 (1957) 497.

[50] M. Bander, Phys. Rev. Lett. 57 (1986) 1825.

151] J.F. Donoghue, Phys. Rev. Lett. 72 (1994) 2996;

H.W. Hamber and S. Liu, Phys. Lett. B 357 (1995) 51;

I.J. Muzinich and S. Vokos, Phys. Rev. D 52 (1995) 3472.

[52] H.W. Hamber and R.M. Williams, Nucl. Phys. B 415 (1994) 463.

[53] H.W. Hamber, Phys. Rev. D 50 (1994) 3932.

[54] K. Fujikawa, in Quantum Gravity and Cosmology, published in the 8th Kyoto Summer Institute (World Scientific, 1985), p. 129.

[55] K. Fujikawa and M. Ninomiya, Nucl. Phys. B 391 (1993) 675.

[56] D. Forster, Nucl. Phys. B 283 (1987) 669; B 291 (1987) 813;

P. Menotti and P. Peirano, Phys. Lett. B 353 (1995) 444; Nucl. Phys. B 473 (1996) 426.

[57| V.A. Kazakov, Phys. Lett. A 119 (1986) 140;

D.V. Boulatov and V.A. Kazakov, Phys. Lett. B 186 (1987) 379.

[58] V.G. Knizhnik, A.M. Polyakov and A.B. Zamolodchikov, Mod. Phys. Lett. A 3 (1988) 819.

[59] F. David, Mod. Phys. Lett. A 3 (1988) 1651;

J. Distler and H. Kawai, Nucl. Phys. B 321 (1989) 509.

[60] M. Vekić, S. Liu and H.W. Hamber, CERN-Th-7177-94 (Feb 1994), Phys. Lett. B 329 (1994) 444; CERN-Th-7355-94 (Jul 1994), Phys. Rev. D 51 (1995) 4287.

[61] W. Beirl and B. Berg, FSU-SCRI-95-39 (May 1995), Nucl. Phys. B 452 (1995) 415.

[62] D. Espriu and J. Matias, Phys. Lett. B 283 (1992) 326;

see also H.C. Ren, Nucl. Phys. B 300 (1988) 531.

[63] W. Bock and J.C. Vink, Nucl. Phys. B 438 (1995) 320. 